\title{
2014 AATS guidelines for the prevention and management of perioperative atrial fibrillation and flutter for thoracic surgical procedures
}

\author{
Gyorgy Frendl, MD, PhD, ${ }^{a}$ Alissa C. Sodickson, MD, ${ }^{a}$ Mina K. Chung, MD, ${ }^{b}$ Albert L. Waldo, MD, PhD, ${ }^{c, d}$ \\ Bernard J. Gersh, MB, ChB, DPhi, ${ }^{e}$ James E. Tisdale, PharmD ${ }^{f}$ Hugh Calkins, MD, ${ }^{\mathrm{g}}$ Sary Aranki, MD, \\ Tsuyoshi Kaneko, MD, ${ }^{\mathrm{h}}$ Stephen Cassivi, MD, ${ }^{\mathrm{i}}$ Sidney C. Smith, Jr, MD, ${ }^{\mathrm{j}}$ Dawood Darbar, MD, ${ }^{\mathrm{k}}$ \\ Jon O. Wee, MD, ${ }^{1}$ Thomas K. Waddell, MD, MSc, PhD,${ }^{\mathrm{m}}$ David Amar, MD, ${ }^{\mathrm{n}}$ and Dale Adler, $\mathrm{MD}^{\circ}$
}

丹 Supplemental material is available online.

\section{PREAMBLE}

Our mission was to develop evidence-based guidelines for the prevention and treatment of perioperative/postoperative atrial fibrillation and flutter (POAF) for thoracic surgical procedures. Sixteen experts were invited by the American Association for Thoracic Surgery (AATS) leadership: 7 cardiologists and electrophysiology specialists, 3 intensivists/ anesthesiologists, 1 clinical pharmacist, joined by 5 thoracic and cardiac surgeons who represented AATS (see Online Data Supplement 1 for the list of members and Online Data Supplement 2 for the conflict of interest declaration online).

\section{Methods of Review}

Members were tasked with making recommendations based on a review of the literature, with grading the quality of the evidence supporting the recommendations, and with assessing the risk-benefit profile for each recommendation. The level of evidence was graded by the task force panel according to standards published by the Institute of Medicine (Table 1). For the development of the guidelines we followed the recommendations of The Institute of Medicine (IOM) 2011 Clinical Practice Guidelines We Can Trust: Standards for Developing Trustworthy Clinical Practice Guidelines; www.iom.edu/ cpgstandards. ${ }^{1}$ Efforts were made to minimize repetition of existing guidelines ${ }^{2-4}$; rather we focused on new information and advances in diagnosis and therapy, and present these current

\footnotetext{
From the Department of Anesthesiology, ${ }^{\text {a }}$ Perioperative Critical Care and Pain Medicine, Brigham and Women's Hospital and Harvard Medical School, Boston, Mass; Department of Cardiovascular Medicine, ${ }^{\mathrm{b}}$ Heart and Vascular Institute, Department of Molecular Cardiology, Lerner Research Institute Cleveland Clinic, Lerner College of Medicine of Case Western Reserve University Cleveland Clinic, Cleveland, Ohio; Division of Cardiovascular Medicine, ${ }^{\mathrm{c}}$ Department of Medicine, Case Western Reserve University, Cleveland, Ohio; Harrington Heart \& Vascular Institute, ${ }^{\mathrm{d}}$ University Hospitals Case Medical Center, Cleveland, Ohio; Division of Cardiovascular Diseases and Internal Medicine, ${ }^{\mathrm{e}}$ Department of Medicine, Mayo Clinic College of Medicine, Rochester, Minn; Department of Pharmacy Practice, ${ }^{f}$ College of Pharmacy, Purdue University and Indiana University School of Medicine, Indianapolis, Ind; Department of Medicine, ${ }^{\mathrm{g}}$ Cardiac Arrhythmia Service, Johns Hopkins University, Baltimore, Md; Division of Cardiac Surgery, ${ }^{\text {h }}$ Department of Surgery, Brigham and Women's Hospital and Harvard Medical School, Boston, Mass; Division of Thoracic Surgery, ${ }^{i}$ Department of Surgery, Mayo Clinic College of Medicine, Rochester, Minn; Center for Heart and Vascular Care, ${ }^{j}$ Department of Medicine, University of North Carolina, Chapel Hill, NC; Division
}

guidelines within the framework of the new IOM recommendations. In order to meet these standards, most societies (American Heart Association and AATS included) initiated the revision ${ }^{2,3}$ of existing guidelines.

Task force subgroups were formed and tasked with preparing a summary of the available literature for each subtopic. Literature searches were conducted using PubMed, focused on articles published since 2000 except in rare circumstances. Both the summaries and original articles were made available to each task force member via a shared electronic folder. The subgroup summaries as well as the original literature were presented and discussed at 9 scheduled teleconferences. The conferences were recorded. Articles were selected for inclusion based on consensus opinion by task force members. Writing groups were formed to develop the draft guidelines for each subtopic, with 3 to 7 members and a leader for each group. Group recommendations were submitted before being presented for discussion and voting at a 1-day face-to-face conference.

Members were specifically asked to assess the applicability of the available evidence to patients undergoing thoracic surgery. All recommendations were subjected to a vote. Acceptance for the final document required greater than $75 \%$ approval of each of the recommendations.

A final draft was prepared by the chairman of the task force and made available in a written form to each member for final comments. Subsequently, the recommendations were posted for public comments for AATS members (via REDCap), and then peer reviewed by outside experts selected by the AATS Council.

of Cardiovascular Medicine, ${ }^{\mathrm{k}}$ Department of Medicine, Arrhythmia Service, Vanderbilt University School of Medicine, Nashville, Tenn; Division of Thoracic Surgery, ${ }^{1}$ Department of Surgery, Brigham and Women's Hospital and Harvard Medical School, Boston, Mass; Division of Thoracic Surgery, ${ }^{\mathrm{m}}$ Department of Surgery, University of Toronto, Toronto, Ontario, Canada; Memorial Sloan-Kettering Cancer Center, ${ }^{\mathrm{n}}$ Department of Anesthesiology and Critical Care Medicine, New York, NY; Division of Cardiovascular Medicine, ${ }^{\circ}$ Department of Medicine, Brigham and Women's Hospital and Harvard Medical School, Boston, Mass.

Disclosures: See Online Data Supplement 2.

Received for publication June 9, 2014; accepted for publication June 10, 2014

Address for reprints: Gyorgy Frendl, MD, PhD, Department of Anesthesiology, Perioperative and Pain Medicine, Brigham and Women's Hospital, CWN-L1, 75 Francis St, Boston, MA 02115 (E-mail: gfrendl@ partners.org).

J Thorac Cardiovasc Surg 2014;148:e153-93 $0022-5223 / \$ 36.00$

Copyright (C) 2014 by The American Association for Thoracic Surgery http://dx.doi.org/10.1016/j.jtcvs.2014.06.036 
The following recommendations are based on the best available evidence from thoracic surgery. When evidence specific to thoracic surgery was not available, we extrapolated from the cardiac surgical literature. In the absence of direct evidence, we present the best expert opinion based on cardiology/cardiac electrophysiology experience and best practices.

An executive summary was prepared for publication in a printed format; this more extensive guideline was prepared for online publication with additional comments, data, and a comprehensive list of references.

\section{AATS Member Survey}

Our survey of the AATS members (results presented in Online Data Supplement 3) indicated the need for a guideline update and identified opportunities for improvement in the areas of prevention, standards for postoperative electrocardiography (ECG) monitoring, and for the possible use of novel oral anticoagulants. When asked how the AATS could help members improve their practices; $29 \%$ of respondents recommended "initiating studies," whereas 58\% recommended that the AATS "issue guidelines" and promote uniform practices.

\section{Target Audience and the Patient Population}

These guidelines are intended for all noncardiac intrathoracic surgeries and esophagectomies, as well as for patients whose risk factors and comorbidities place them at intermediate to high risk for POAF, independent of the procedure. In assessing the patient's risk for POAF, it must be noted that the risks posed by the procedure and by patient factors/comorbidities will likely be additive, if not synergistic. Therefore, these factors should be evaluated in combination during the preoperative assessment.

The target audience includes not only thoracic surgeons and anesthesiologists but all providers who participate in the care of thoracic surgical patients.

The following novel information is included in this 2014 document: (1) standardized definitions for atrial fibrillation (AF) and (2) recommendations for: (a) ECG monitoring, (b) postdischarge management, (c) use of the new class of novel oral anticoagulants (NOAC); and (d) obtaining cardiology consultation. In addition, flow diagrams summarize the strategies for acute and chronic management. Specific drug recommendations and dosing tables are also included.

\section{EPIDEMIOLOGY OF POAF, ITS IMPACT ON OUTCOMES, COST, AND MORBIDITY}

$\mathrm{AF}$, the most common sustained arrhythmia after pulmonary and esophageal surgery, is a major, potentially preventable, adverse outcome. POAF peaks on postoperative days 2 to 4 , and $90 \%$ to $98 \%$ of new-onset POAF resolves within 4 to 6 weeks. Postoperative atrial fibrillation has multiple negative implications. In the acute setting, the tachyarrhythmia can lead to hemodynamic instability, necessitating prompt intervention. A sustained increased heart rate can result in heart failure, a less common but clinically devastating situation, the incidence of which is not reported in the literature.

The incidence of POAF varies widely based on the intensity of surgical stress (Table 2, $A^{5-17}$ ) and patient characteristics (Table 2, $B^{5,6,8,10,18-20}$ ). Some of the risk factors for AF such as hypertension, obesity, and smoking, are modifiable, whereas others, such as older age, Caucasian ancestry, and male sex, are not.

Thromboembolic events such as stroke or acute limb ischemia are the most serious and feared consequences of AF. Studies have reported a wide range of the incidence of stroke related to POAF, although the risk for cardiac and thoracic surgery seems to be $50 \%$ to $200 \%$ higher than for general surgery. ${ }^{10,21,22}$

Many studies show an increase in mortality in patients with POAF $^{6,10,15,16,23,24}$ although some studies have not shown such an effect. ${ }^{13,25}$ Given that patients with other significant comorbidities or who are undergoing more complex operations are more likely to experience POAF, it is unclear to what extent the arrhythmia itself contributes to mortality. It is feasible that the contribution of POAF to mortality is more significant for those patients with fewer other comorbidities, however this independent effect is more difficult to measure and has not been well reported in the literature.

POAF is associated with longer intensive care unit and hospital stays, increased morbidity (including strokes/new central neurologic events) with incidence of $1.3 \%$ $1.7 \%^{10,21,22}$; and mortality (up to $5.6 \%-7.5 \%$; RR 1.7 $3.4^{6,10,15,16,23,24}$ ), as well as higher resource utilization. ${ }^{6,26}$

Multiple studies have consistently demonstrated an increase in length of hospital stay in patients who develop POAF, generally by a mean of 2 to 4 days. ${ }^{5,6,10,15,16,19,23,24}$ An analysis of the Society of Thoracic Surgeons (STS) database by Onatis and colleagues ${ }^{10}$ demonstrated that, in patients undergoing lobectomy or greater resection for lung cancer, the presence of POAF lengthened hospital stay by a median of 3 days. The cost of hospitalization is likewise increased for patients who develop POAF, with an increase reported in the literature anywhere from $30 \%$ to $68 \%$. $^{5,6,23}$ To some extent, this increase reflects comorbid conditions that occur along with POAF, but POAF itself is associated with an increase in cost. Vaporciyan and colleagues ${ }^{5}$ found that for patients who developed POAF without any other complications, the cost of care increased by more than US $\$ 6000$, representing a greater than $30 \%$ increase.

\section{THE POSSIBLE MECHANISMS OF POAF AFTER THORACIC SURGERY}

The mechanisms that initiate and sustain AF, including POAF, are complex and require both a vulnerable atrial substrate $^{27}$ and a trigger to initiate AF (Table 3). Today they remain incompletely understood. The role of triggers 
TABLE 1. Size of treatment effect and level of evidence for its impact

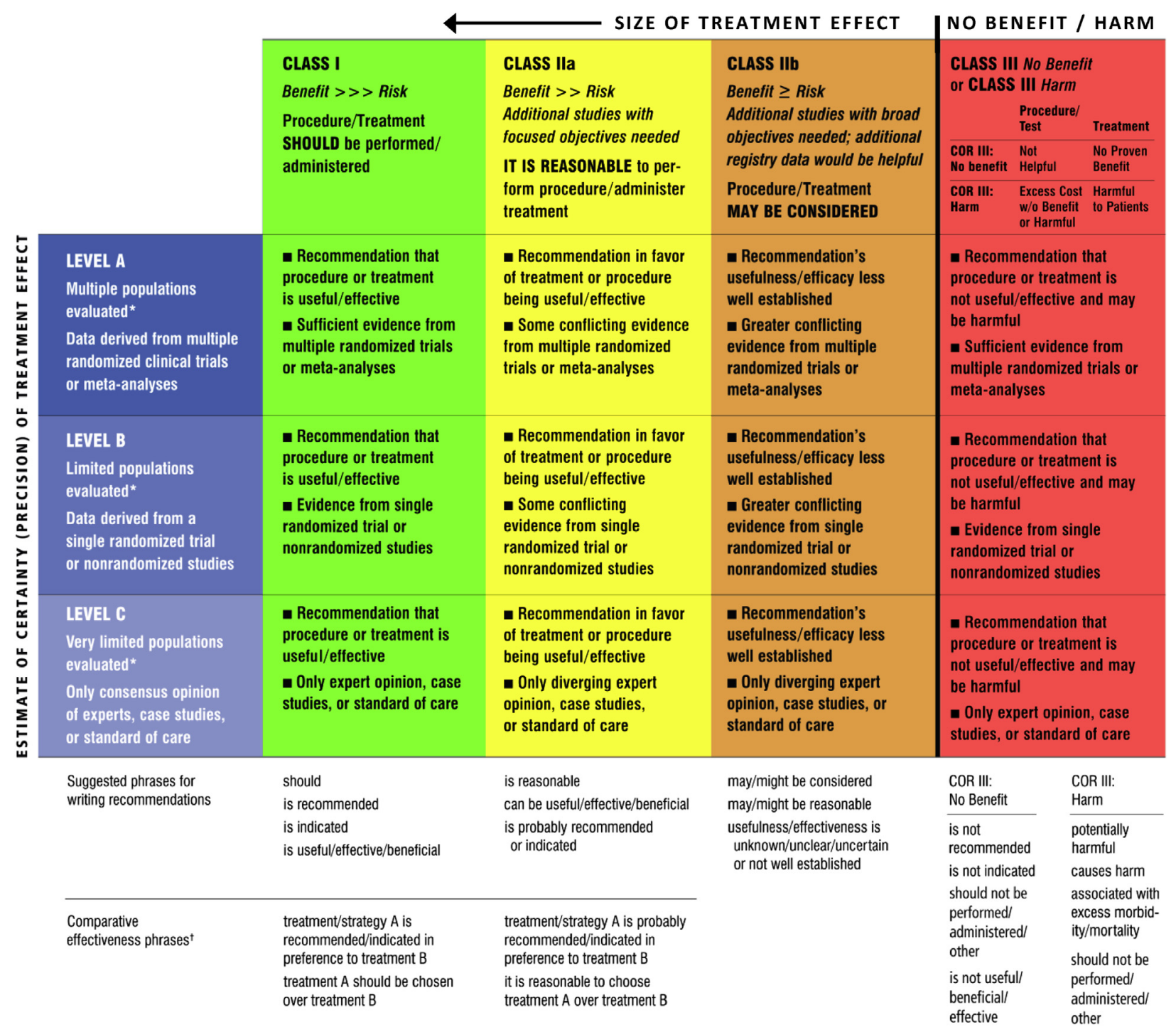

Schema used to guide the grading of available published evidence and the expected effect of the interventions for their impact on patient outcomes (the arrow indicates the direction of increased effect size). COR, Class of recommendation.

from the pulmonary veins and other atrial sites initiating $\mathrm{AF}^{28}$ is well appreciated. However, it remains to be understood why they occur and what exact mechanisms are essential for their propagation. The identified risk factors for the development of sustained POAF are mostly identical to those known to make the atrium vulnerable to development of $\mathrm{AF}$ in the nonsurgical setting. They include several risk factors that are associated with atrial fibrosis, such as increasing age, atrial dilatation, myocardial ischemia, volume overload, and a history of heart failure. ${ }^{29-31}$ They also include risk factors such as increased norepinephrine levels and increased vagal tone, both of which shorten atrial wavelength, the latter known to increase atrial vulnerability to $A F .{ }^{32}$ Both adrenergic and vagal stimulation can promote triggers that initiate $\mathrm{AF}^{33}$ In addition, surgical procedures are associated with local or systemic inflammation (such as pericarditis), an important risk factor affecting the vulnerability of the atrial substrate to POAF. ${ }^{34}$ The extent of pulmonary resection is another important risk factor for development of POAF. ${ }^{7}$ The development of POAF is likely to involve some or all of these mechanisms.

Insight into the mechanism of POAF can be gained by examining what prophylactic therapies decrease the rate of POAF occurrence after thoracic surgery. Higher norepinephrine levels were found in patients on preoperative $\beta$-blockers who had their $\beta$-blocker therapy interrupted than in patients not receiving a $\beta$-blocker at all. This was associated with a significantly higher incidence of POAF. ${ }^{30,35}$ 
TABLE 2, A. Risk stratification of thoracic surgical procedures for their risk of POAF

\begin{tabular}{|c|c|c|c|}
\hline \multirow[b]{2}{*}{ Type of procedures } & \multicolumn{3}{|c|}{ Risk of POAF by surgical procedures } \\
\hline & $\begin{array}{l}\text { Low risk procedures } \\
(<\mathbf{5} \% \text { incidence })\end{array}$ & $\begin{array}{c}\text { Intermediate risk procedures } \\
(5 \%-15 \% \text { incidence }) \\
\end{array}$ & $\begin{array}{l}\text { High risk procedures } \\
(>15 \% \text { incidence })\end{array}$ \\
\hline \multicolumn{4}{|c|}{ Intrathoracic/airway procedures } \\
\hline Minor procedures & $\begin{array}{l}\text { Flexible bronchoscopy with and } \\
\text { without biopsy } \\
\text { Photodynamic therapy } \\
\text { Tracheal stenting } \\
\text { Placement of thoracostomy tube or } \\
\text { PleurX catheter (CareFusion } \\
\text { Corporation, San Diego, Calif) } \\
\text { Pleuroscopy, pleurodesis, } \\
\text { decortication }\end{array}$ & & \\
\hline $\begin{array}{l}\text { Procedures with } \\
\text { moderate stress }\end{array}$ & $\begin{array}{l}\text { Tracheostomy } \\
\text { Rigid bronchoscopy } \\
\text { Mediastinoscopy } \\
\text { Thoracoscopic wedge resection } \\
\text { Bronchoscopic laser surgery }\end{array}$ & Thoracoscopic sympathectomy & \\
\hline Major procedures & & Segmentectomy ${ }^{5,6}$ & $\begin{array}{l}\text { Resection of anterior mediastinal } \\
\text { mass } \\
\text { Thoracoscopic lobectomy } \\
\text { Open thoracotomy for lobectomy }{ }^{5-11} \\
\text { Tracheal resection and } \\
\text { reconstruction/carinal resection } \\
\text { Pneumonectomy } \\
\text { Pleurecti-13 } \\
\text { Volume reduction/bullectomy } \\
\text { Bronchopleural fistula repair } \\
\text { Clagett window } \\
\text { Lung transplantation } \\
\text { I-16 }^{8}\end{array}$ \\
\hline Esophageal procedures & $\begin{array}{l}\text { Esophagoscopy/PEG/esophageal } \\
\text { dilation and/or stenting }\end{array}$ & $\begin{array}{l}\text { Laparoscopic Nissen fundoplication/ } \\
\text { myotomy } \\
\text { Zenker diverticulectomy }\end{array}$ & Esophagectomy ${ }^{5,8,17}$ \\
\hline Other procedures & & & Pericardial window \\
\hline
\end{tabular}

Thoracic surgical procedures were divided into low $(<5 \%)$, moderate $(5 \%-15 \%)$ and high $(>15 \%)$ risk groups based on their expected incidence of POAF in order to facilitate the preoperative risk stratification of patients. $P O A F$, Postoperative atrial fibrillation; $P E G$, percutaneous endoscopic gastrostomy.

Diltiazem therapy initiated in the early postoperative period has been found to significantly reduce the rate of POAF. $^{36}$ This is believed to be related to its effects of decreasing pulmonary vascular resistance. It is known that pulmonary hypertension and dilatation of the right side of the heart are associated with an increased incidence of POAF. ${ }^{29}$ There is also the possibility that as a systemic vasodilator, diltiazem could reduce preload and left atrial pressures. ${ }^{33}$ The data on use of verapamil have been inconsistent with regard to decreasing the incidence of POAF. ${ }^{37}$ Magnesium has been consistently shown to decrease the incidence of POAF after cardiac surgery, and the only prospective, randomized study on patients undergoing thoracic surgery also showed a significant decrease in the incidence of POAF. ${ }^{35}$ The reason for its effectiveness is uncertain.

In the presence of a vulnerable substrate, additional electrophysiologic abnormalities (drivers) will sustain AF.

\section{RECOMMENDATIONS AND REASONING}

\section{Recommend the Use of the Following Definitions for the Diagnosis of POAF Class I}

1.1. Electro-physiologic definition/diagnosis: ECG recordings ( 1 or more ECG leads) that demonstrate the presence of characteristic ECG features of AF lasting at least for 30 seconds or for the duration of the ECG recording (if shorter than 30 seconds) ${ }^{2,38}$ (level of evidence LOE C). Clinical symptoms may include hypotension, dizziness, decreased urinary output, fatigue, and so on.

1.2. Clinical definition/diagnosis: clinically significant POAF (Table 4) is AF in the (intra- and) postoperative setting that requires treatment with rate or rhythm control agents, or requires anticoagulation, and/or extends the duration of hospitalization (LOE C). Clinical 
TABLE 2, B. Known patient risk factors for and comorbidities that increase the risk of POAF

\begin{tabular}{|c|c|}
\hline Risk factors and comorbidities & Thoracic surgery references \\
\hline \multicolumn{2}{|l|}{ Modifiable risk factors } \\
\hline Hypertension & $8,10,18$ \\
\hline MI & 19 \\
\hline \multicolumn{2}{|l|}{ VHD } \\
\hline Heart failure & $5,6,20$ \\
\hline Obesity & 10 \\
\hline \multicolumn{2}{|l|}{ Obstructive sleep apnea } \\
\hline \multicolumn{2}{|l|}{ Smoking } \\
\hline \multicolumn{2}{|l|}{ Exercise } \\
\hline \multicolumn{2}{|l|}{ Alcohol use } \\
\hline \multicolumn{2}{|l|}{ Hyperthyroidism } \\
\hline \multicolumn{2}{|l|}{ Increased pulse pressure } \\
\hline \multicolumn{2}{|l|}{ Mitral regurgitation } \\
\hline \multicolumn{2}{|l|}{ LVH } \\
\hline \multicolumn{2}{|l|}{ Increased LV wall thickness } \\
\hline \multicolumn{2}{|l|}{ Nonmodifiable risk factors } \\
\hline Increasing age & $5,6,8,10,19,20$ \\
\hline African American (protective factor) & 10 \\
\hline \multicolumn{2}{|l|}{ Family history } \\
\hline \multicolumn{2}{|l|}{ Genetic variants } \\
\hline Male sex & $5,8,10,20$ \\
\hline History of arrythmias & 5,6 \\
\hline \multicolumn{2}{|c|}{$\begin{array}{l}\text { Derived from the } 2014 \text { American Heart Association Atrial Fibrillation Guidelines } \\
\text { and relevant literature for thoracic surgery. Patient risk factors and comorbidities } \\
\text { that were shown to increase the risk of atrial fibrillation (AF) are listed. Much of } \\
\text { this information was extracted from the general population, thoracic surgery-specific } \\
\text { references are listed when available. These risk factors/comorbidities should be as- } \\
\text { sessed in conjunction with the procedure-related risks of AF in order to determine } \\
\text { the true risk of POAF. } M I \text {, Myocardial infarction; } V H D \text {, valvular heart disease; } L V \text {, } \\
\text { left ventricle; } L V H \text {, left ventricular hypertrophy. }\end{array}$} \\
\hline
\end{tabular}

symptoms may include hypotension, dizziness, decreased urinary output, fatigue, and so on.

We recommend that both electrophysiologically documented $\mathrm{AF}$ and clinically diagnosed $\mathrm{AF}$ be included in the clinical documentation and reported in the clinical trials/studies.

\section{Physiologic (ECG) Monitoring of Patients at Risk for POAF}

Recommendations for ECG monitoring of patients at risk for POAF are presented in Table 5.

\section{Class I}

2.1. Patients should be monitored with continuous ECG telemetry postoperatively for 48 to 72 hours (or less if their hospitalization is shorter) if:

2.1.1. They are undergoing procedures that pose intermediate $(5 \%-15 \%$ expected incidence of $\mathrm{AF})$ or high $(>15 \%)$ risk for the development of postoperative $\mathrm{AF}$ or have significant additional risk factors $\left(\mathrm{CHA}_{2} \mathrm{DS}_{2}\right.$-VASc $\geq 2$ ) for stroke (LOE C).

2.1.2. They have a history of preexisting or periodic recurrent AF before their surgery. These patients
TABLE 3. Probable mechanisms contributing to POAF

Clinically meaningful AF requires the presence of both a trigger and a vulnerable atrial substrate

Atrial substrate changes that facilitate AF

Sympathetic or parasympathetic stimulation

Atrial dilation or acute atrial stretch

Pericarditis

Fibrosis

Inhomogeneous dispersion of conduction abnormalities

Short wavelength (conduction velocity $\times$ ERP)

Other (like inflammation and oxidative stress)

In addition, a driver(s) is thought to be needed to sustain $\mathrm{AF}$ in the vulnerable substrate

Rapidly firing ectopic focus (atrial or other)

Reentrant circuit(s) of short cycle length (ordered reentry)

Potential role, if any, of multiple reentrant wavelets (random reentry)

$A F$, Atrial fibrillation; $E R P$, effective refractory period.

should also receive ECG monitoring in the immediate preoperative period if procedures (eg, epidural catheter or other regional anesthesia

Class IIa blocks) are performed (LOE C).

2.2. Not using routine ECG telemetry is reasonable for patients who undergo low-risk $(<5 \%$ expected incidence of $\mathrm{AF}$ ) procedures, and have neither a previous history of $\mathrm{AF}$ nor significant risk for stroke (based on $\mathrm{CHA}_{2} \mathrm{DS}_{2}$-VASc score), and have no relevant comorbidities (such as heart failure or previous stroke) (LOE C).

\section{Class I}

2.2.1. If patients exhibit clinical signs of possible $\mathrm{AF}$ while not monitored with telemetry, ECG recordings to diagnose POAF and ongoing telemetry to monitor the period of $\mathrm{AF}$ should be immediately implemented (LOE C).

\section{Rate Control and Antiarrhythmic Drugs,} Mechanism of Action, Side Effects, and Limitations

A detailed description of the drugs used for the management of rate (Table 6) or rhythm control (Table $7^{39}$ ), their mechanism of action, side effects, and limitations are discussed here. Dosing information is also presented in Tables 6 and 7.

\section{Recommendation}

\section{Class IIa}

3.1. To optimize the efficacy and safety of amiodarone, it is reasonable to exercise caution when selecting its doses or intravenous versus oral route, because cases of acute respiratory distress syndrome (ARDS) have been reported following pneumonectomy with cumulative intravenous doses more than $2150 \mathrm{mg}^{40}$ (LOE C). 
TABLE 4. Recommended definitions for the diagnosis of POAF

\begin{tabular}{|c|c|c|}
\hline Definitions & & COR \\
\hline $\begin{array}{l}\text { Electrophysiologic } \\
\text { definition/diagnosis }\end{array}$ & $\begin{array}{l}\text { ECG recordings ( } 1 \text { or more ECG } \\
\text { leads) with ECG features of AF } \\
\text { lasting at least for } 30 \text { seconds or } \\
\text { for the duration of the ECG } \\
\text { recording (if }<30 \text { seconds) } \\
\text { (LOE C) }\end{array}$ & I \\
\hline $\begin{array}{l}\text { Clinical definition/ } \\
\text { diagnosis }\end{array}$ & $\begin{array}{l}\text { Clinically significant POAF: } \\
\text { intra- and postoperative AF } \\
\text { requiring treatment, or } \\
\text { anticoagulation, and/or } \\
\text { extending the duration of } \\
\text { hospitalization (LOE C) }\end{array}$ & I \\
\hline
\end{tabular}

These measures should be included in the clinical documentation and reported in the clinical trials/studies. POAF, Postoperative atrial fibrillation; $E C G$, electrocardiography; $C O R$, class of recommendation; $L O E$, level of evidence; $A F$, atrial fibrillation.

\section{Reasoning}

\subsection{Rate control agents: their mechanisms of action and side effects}

\subsection{1. $\beta$-Blockers}

$\beta$-Blockers are Vaughan Williams class II antiarrhythmic agents that inhibit sympathetic nervous system activity and slow the rate of phase IV

TABLE 5. Recommendations for physiologic (ECG) monitoring

\begin{tabular}{ll}
\hline Recommendations for monitoring & COR \\
\hline Patients should be monitored with continuous ECG & I \\
telemetry postoperatively for $48-72 \mathrm{~h}$ (or less if their & \\
hospitalization is shorter) if: & \\
- they are undergoing procedures that pose high \\
$(>15 \%$ expected incidence of AF) or intermediate \\
- $5 \%-15 \%$ ) risk for POAF or \\
they have significant additional risk factors \\
(CHA $\mathrm{AS}_{2}$-VASc $>2$ ) for stroke (LOE C) \\
they have a history of preexisting or periodic \\
recurrent AF before their surgery \\
These patients should also receive ECG monitoring \\
in the immediate preoperative period if procedures \\
(epidural catheter, regional anesthesia blocks, and \\
so forth) are performed (LOE C) \\
Not using routine ECG telemetry is reasonable for \\
patients who \\
- undergo low risk surgery (<5\% expected incidence \\
of AF) and \\
- had no previous history of AF, or \\
- have no significant risk for stroke and \\
previous stroke) (LOE C) \\
not monitored with ECG telemetry, ECG \\
recordings to diagnose POAF and continuous \\
telemetry to monitor the period of AF should be \\
immediately implemented (LOE C)
\end{tabular}

$E C G$, Electrocardiography; $C O R$, class of recommendation; $A F$, atrial fibrillation; $P O A F$, postoperative atrial fibrillation; $L O E$, level of evidence. repolarization, thus slowing the discharge from the sinus node. ${ }^{41}$ This antiadrenergic activity inhibits the renin-angiotensin-aldosterone system, inhibits apoptosis, and reduces hyperphosphorylation of calcium-releasing channels. ${ }^{42}$ Metoprolol and atenolol are relatively selective $\beta-1$ receptor antagonists (primarily affecting cardiac tissue) and in moderate doses have less effect on the $\beta$ - 2 receptors in smooth muscle cells in the vasculature and bronchial tree. Propranolol and esmolol are nonselective, and carvedilol is nonselective and possesses $\alpha$-receptor blocking activity.

Intravenous administration of metoprolol, propranolol, and esmolol reduces ventricular response in patients with $\mathrm{AF}$ within 5 minutes of administration, ${ }^{43}$ and both intravenous and oral regimens attain resting and exercise rate control, variably defined, in $68 \%$ to $75 \%$ of patients. $^{44-47}$ Rate-lowering efficacy varies with acuity and cardiac function and is enhanced with digoxin. ${ }^{45,46}$

The major adverse effects of $\beta$-blockers are bronchospasm in patients with asthma, particularly if the asthma is not well controlled; worsening of symptoms in patients with severe peripheral arterial disease; hypotension; and worsening of

TABLE 6. Commonly used rate control agents

\begin{tabular}{|c|c|c|}
\hline Drug & Recommended doses & $\begin{array}{l}\text { Significant limitations } \\
\text { and known side effects }\end{array}$ \\
\hline Diltiazem & $\begin{array}{l}0.25 \mathrm{mg} / \mathrm{kg} \text { IV loading dose } \\
\text { over } 2 \mathrm{~min} \text {, then } 5-15 \mathrm{mg} / \\
\text { h IV continuous infusion }\end{array}$ & $\begin{array}{l}\text { Hypotension } \\
\text { Bradycardia } \\
\text { Heart failure exacerbation }\end{array}$ \\
\hline Digoxin & $\begin{array}{l}0.25 \mathrm{mg} \text { IV repeated every } \\
2-4 \mathrm{~h} \text { to a maximum dose } \\
\text { of } 1.5 \mathrm{mg} \text { over } 24 \mathrm{~h}\end{array}$ & $\begin{array}{l}\text { Nausea, vomiting, anorexia } \\
\text { Confusion } \\
\text { AV block } \\
\text { Ventricular arrhythmias } \\
\text { Accumulates in acute } \\
\text { kidney injury/chronic } \\
\text { kidney disease }\end{array}$ \\
\hline Esmolol & $\begin{array}{l}500 \mu \mathrm{g} / \mathrm{kg} \text { IV bolus over } 1 \\
\mathrm{~min}, \text { then } 50-300 \mu \mathrm{g} / \mathrm{kg} / \\
\text { min IV continuous } \\
\text { infusion }\end{array}$ & $\begin{array}{l}\text { Bradycardia } \\
\text { Hypotension } \\
\text { Bronchospasm } \\
\text { Heart failure exacerbation }\end{array}$ \\
\hline Metoprolol & $\begin{array}{c}2.5-5.0 \mathrm{mg} \text { IV bolus over } 2 \\
\text { min; maximum } 3 \text { doses }\end{array}$ & $\begin{array}{l}\text { Bradycardia } \\
\text { Hypotension } \\
\text { Bronchospasm } \\
\text { Heart failure exacerbation }\end{array}$ \\
\hline Amiodarone & $\begin{array}{l}\text { 150-300 mg IV over } 1 \mathrm{~h}, \\
\text { followed by } 10-50 \mathrm{mg} / \mathrm{h} \\
\text { IV continuous infusion } \\
\text { over } 24 \mathrm{~h}\end{array}$ & $\begin{array}{l}\text { Bradycardia } \\
\text { QT interval prolongation } \\
\text { Pulmonary toxicity has not } \\
\text { been demonstrated at this } \\
\text { dose }\end{array}$ \\
\hline
\end{tabular}

Detailed information in section 3 and in references 2 and 3. IV, Intravenous; $A V$, atrioventricular. 
TABLE 7. Commonly used antiarrhythmic agents

\begin{tabular}{|c|c|}
\hline Drug & Recommended doses \\
\hline Procainamide & $\begin{array}{l}\text { Conversion to sinus rhythm: } 20-50 \mathrm{mg} / \mathrm{min} \text { IV } \\
\text { continuous infusion until AF terminated, } \\
\text { hypotension occurs, or QRS duration prolonged by } \\
50 \% \text {, or cumulative total dose of } 15 \mathrm{mg} / \mathrm{kg} \text { reached } \\
\text { Alternative dose: } 100 \mathrm{mg} \text { IV every } 5 \mathrm{~min} \text { until AF } \\
\text { terminated or other conditions as listed above are } \\
\text { met }\end{array}$ \\
\hline
\end{tabular}

Flecainide

Conversion to sinus rhythm: 200-300 mg single oral dose

Maintenance of sinus rhythm: 50-150 orally once every $12 \mathrm{~h}$

Propafenone

Conversion to sinus rhythm: $450-600 \mathrm{mg}$ single oral dose

Maintenance of sinus rhythm: $150-300 \mathrm{mg}$ orally every $8 \mathrm{~h}$ (immediate release); $225-425 \mathrm{mg}$ orally every $12 \mathrm{~h}$ (extended release)

Amiodarone

Prophylaxis: $300 \mathrm{mg}$ IV bolus, then $600 \mathrm{mg}$ orally twice daily for 3-5 d

Treatment: $150 \mathrm{mg}$ IV over $10 \mathrm{~min}$; then $1 \mathrm{mg} / \mathrm{min} \mathrm{IV}$ continuous infusion for $6 \mathrm{~h}$; the $0.5 \mathrm{mg} / \mathrm{min} \mathrm{IV}$ continuous infusion for $18 \mathrm{~h}$ or change to oral administration at $100-400 \mathrm{mg}$ daily

Dofetilide

Ibutilide

Conversion to sinus rhythm:

Weight $\geq 60 \mathrm{~kg}: 1 \mathrm{mg}$ IV administered over $10 \mathrm{~min}$

Weight $<60 \mathrm{~kg}: 0.01 \mathrm{mg} / \mathrm{kg}$ IV administered over $10 \mathrm{~min}$ postoperative setting; may take 2-3 d to convert to normal sinus rhythm, which would require commitment to anticoagulation

Maintenance of sinus rhythm: calculated $\mathrm{CrCl} 20-40$ $\mathrm{mL} / \mathrm{min}: 125 \mu \mathrm{g}$ orally once every $12 \mathrm{~h}$

Calculated $\mathrm{CrCl} 40-60 \mathrm{~mL} / \mathrm{min}: 250 \mu \mathrm{g}$ orally once every $12 \mathrm{~h}$

\section{Significant limitations and}

known side effects

Ref

Hypotension

QT interval prolongation

Torsades de pointes

Contraindicated in patients with heart failure with reduced left ventricular ejection fraction

Contraindicated in patients with pretreatment QTc interval $>470 \mathrm{~ms}$ (men) or $480 \mathrm{~ms}$ (women)

Dizziness

Blurred vision

Sinus bradycardia

AV block

Contraindicated in patients with heart failure with reduced left ventricular ejection fraction

Contraindicated in patients with coronary artery disease/structural heart disease

Dizziness

Blurred vision

Sinus bradycardia

AV block

Contraindicated in patients with heart failure with reduced left ventricular ejection fraction

Contraindicated in patients with coronary artery disease/structural heart disease

Bradycardia

QT interval prolongation

Pulmonary toxicity has not been demonstrated at this dose

Bradycardia

Hypotension

QT interval prolongation

Pulmonary toxicity has occurred at cumulative IV doses $>2150 \mathrm{mg}$ in patients undergoing pneumonectomy

QT interval prolongation

Torsades de pointes

Risk of torsades de pointes is greater in patients with heart failure

Dose adjustment is important in patients with acute kidney injury or chronic kidney disease

Contraindicated in patients with calculated $\mathrm{CrCl}<20$ $\mathrm{mL} / \mathrm{min}$

Contraindicated in patients with pretreatment QTc interval $>470 \mathrm{~ms}$ (men) or $480 \mathrm{~ms}$ (women)

Monitor ECGs $2 \mathrm{~h}$ after doses, telemetry for at least $3 \mathrm{~d}$

QT interval prolongation

Torsades de pointes

Risk of torsades de pointes greater in patients with heart

Corvert prescribing information 2006; Pfizer, Inc 
TABLE 7. Continued

\begin{tabular}{|c|c|c|c|}
\hline Drug & Recommended doses & $\begin{array}{c}\text { Significant limitations and } \\
\text { known side effects }\end{array}$ & Ref \\
\hline Sotalol & $\begin{array}{l}\text { If the } \mathrm{AF} \text { does not terminate within } 10 \mathrm{~min} \text { of } \\
\text { completion of the first infusion, a second dose of } \\
\text { equal strength may be administered IV over } 10 \mathrm{~min} \\
\text { Not indicated for maintenance of sinus rhythm } \\
\text { Maintenance of sinus rhythm: } 40-160 \mathrm{mg} \text { orally every } \\
12 \mathrm{~h} \\
\text { Dosing interval should be adjusted in patients with } \\
\text { acute kidney injury or chronic kidney disease: } \\
\text { If the calculated } \mathrm{CrCl} \text { is } 30-59 \mathrm{~mL} / \mathrm{min} \text { : administer } \\
\text { every } 24 \mathrm{~h} \\
\text { If the calculated } \mathrm{CrCl} \text { is } 10-29 \mathrm{~mL} / \mathrm{min} \text { : administer } \\
\text { every } 36-48 \mathrm{~h}\end{array}$ & $\begin{array}{l}\text { Nonsustained ventricular tachycardia } \\
\text { Sinus pauses after AF conversion } \\
\text { Contraindicated in patients with pretreatment QTc } \\
\text { interval }>470 \mathrm{~ms} \text { (men) or } 480 \mathrm{~ms} \text { (women) } \\
\text { Sinus bradycardia } \\
\text { AV block } \\
\text { QT interval prolongation } \\
\text { Torsades de pointes } \\
\text { Heart failure exacerbation } \\
\text { Risk of torsades de pointes greater in patients with heart } \\
\text { failure } \\
\text { Bronchospasm } \\
\text { Dose adjustment is important in patients with acute } \\
\text { kidney injury or chronic kidney disease } \\
\text { Use with extreme caution in patients with calculated } \\
\text { CrCl }<10 \mathrm{~mL} / \mathrm{min} \text { and in patients undergoing } \\
\text { hemodialysis } \\
\text { Contraindicated in patients with pretreatment QTc } \\
\text { interval }>470 \mathrm{~ms} \text { (men) or } 480 \mathrm{~ms} \text { (women) }\end{array}$ & 2,3 \\
\hline
\end{tabular}

$I V$, Intravenous; $A F$, atrial fibrillation; $A V$, atrioventricular; $C r C l$, creatinine clearance; $E C G$, electrocardiography.

heart failure symptoms in patients with decompensated heart failure with reduced ejection fraction. Intravenous $\beta$-blockers should not be used in patients with suspected accessory conduction pathways. $^{2,3,43}$ Profound bradycardia can result from acute concomitant administration of $\beta$-blockers and diltiazem or verapamil.

\subsubsection{Diltiazem}

Diltiazem is a nondihydropyridine calcium channel antagonist and class IV Vaughan Williams agent. Diltiazem inhibits L-type calcium channels in vascular and conduction tissue, and especially in nodal tissue. ${ }^{48}$ In addition, diltiazem affects the transient outward and ultrarapid delayed rectifier potassium currents in atrial myocytes. Intravenous diltiazem administered as a bolus and continuous infusion can control ventricular response in $70 \%$ to $90 \%$ of patients with the recent-onset of AF. The onset of action of diltiazem is 2 to 7 minutes. ${ }^{43,49,50}$

Oral treatment with diltiazem in the Atrial Fibrillation Follow-Up Investigation of Rhythm Management (AFFIRM) trial was efficacious in controlling rest and exercise heart rate in approximately $60 \%$ of patients, and in $66 \%$ and $79 \%$ of patients, respectively, when combined with digoxin. $^{45}$

Diltiazem can worsen heart failure in patients with reduced ejection fraction, and can cause important gastrointestinal adverse effects including ileus.
Diltiazem must be used cautiously, especially acutely, in patients concomitantly receiving $\beta$-blockers, and is contraindicated in patients with an accessory pathway. ${ }^{43}$

\subsubsection{Digoxin}

Digoxin inhibits sodium potassium adenosine triphosphatase (ATPase), thereby increasing intracellular sodium concentration leading to increased intracellular calcium concentrations. In addition, digoxin administration is associated with an increase in baroreceptor sensitivity disproportionate to hemodynamic improvement, and imparts vagomimetic (parasympathetic) effects. The vagomimetic effects of digoxin occur at low serum concentrations and contribute to decreasing sinus and atrioventricular (AV) nodal conduction. At higher serum concentrations, the parasympathetic effects actually shorten the refractory period of nonnodal specialized conduction tissue. ${ }^{51}$

The onset of action of digoxin after intravenous administration of 0.5 to $0.75 \mathrm{mg}$ bolus doses is 30 minutes to 2 hours. ${ }^{52,53}$ With additional intravenous bolus doses of $0.25 \mathrm{mg}$ every 2 to 6 hours after the first dose, up to a total dose within 24 hours of 1.25 to $1.5 \mathrm{mg}, 75 \%$ of patients with $\mathrm{AF}$ can achieve rate control at rest. ${ }^{49,50}$ Exercise rate control is achieved much less frequently, except when digoxin is administered con-comitantly with a $\beta$-blocker or calcium channel blocker. ${ }^{45}$ 
Digoxin should not be administered to patients with suspected accessory pathways or obstructive hypertrophic cardiomyopathy. The potential for digoxin toxicity, including accelerated junctional rhythm, accelerated ventricular escape rhythms (sometimes heralded by regularization of the longest R-R intervals), nausea, and visual symptoms is increased in the presence of hypokalemia, hypomagnesemia, hypercalcemia, and concomitant therapy with amiodarone, dronedarone or verapamil. ${ }^{2,3,43}$ Propensity matched comparisons in the AFFIRM trial do not suggest an increase in mortality associated with chronic digoxin use. ${ }^{54}$

\subsubsection{Amiodarone}

Amiodarone is a Vaughan Williams class III agent that inhibits inward potassium current, prolonging the action potential. However, amiodarone also has properties that could place it in the other 3 Vaughan Williams classes. It has antisympathetic and calcium-blocking activity that leads to important effects on the sinoatrial (SA) and AV nodes, and the drug also has sodium channel-inhibiting properties that increases the threshold for depolarization. $^{55,56}$

Intravenous amiodarone, administered as a bolus and continuous infusion, has an effect on heart rate within 4 hours that is similar to intravenous diltiazem and intravenous digoxin, and improves ventricular rate in $74 \%$ of patients with AF by 24 hours. ${ }^{50}$ Oral amiodarone can require days for effective rate control to occur. Chronic oral amiodarone therapy for rate control can have effects similar to those of digoxin. ${ }^{2,3,57}$

Amiodarone is highly lipophilic, and intravenous administration may exert effects that are different from those following oral administration. Intravenous amiodarone can be associated with $\mathrm{AV}$ block, vasodilation, and hypotension. Intravenous amiodarone should not be used in patients who have a suspected accessory pathway. ${ }^{2,3}$ Pulmonary toxicity associated with high-dose intravenous amiodarone is discussed in section 3.5.1.

Chronic administration of oral amiodarone can be associated with pulmonary, hepatic, thyroid, neurologic, cutaneous, and ocular toxicities. ${ }^{43}$ Amiodarone inhibits the metabolism of warfarin and inhibits elimination of the new oral anticoagulants. Amiodarone administration can restore sinus rhythm so patients who receive it after 24 to 48 hours of $\mathrm{AF}$ require anticoagulation.
3.3. Antiarrhythmic medications (mechanisms of action, side effects)

3.3.1. Amiodarone (see section 3.2.4)

3.3.2. Flecainide

Flecainide is a Vaughan Williams class IC antiarrhythmic agent that is a potent inhibitor of fast sodium conduction. ${ }^{41}$ Consequently, flecainide decreases the maximum upstroke velocity and amplitude of atrial, ventricular, and Purkinje fiber action potentials. ${ }^{58}$ Flecainide may also inhibit $\mathrm{I}_{\mathrm{Kr}}$ current, and prolongs the duration of atrial and ventricular action potential. In patients without structural heart disease, oral flecainide is relatively well tolerated; adverse effects include dizziness (15\%-20\%) and visual abnormalities, including blurred vision and difficulty in focusing (up to $15 \%$ ), which can usually occur during dose uptitration. ${ }^{59}$ However, in patients with structural heart disease, flecainide is associated with more severe adverse effects. Flecainide is associated with ventricular proarrhythmia in this population; this proarrhythmia is not torsades de pointes (TdP), but rather monomorphic ventricular tachycardia. This proarrhythmia was the likely cause of death associated with flecainide (and encainide) in the Cardiac Arrhythmia Suppression Trial (CAST) ${ }^{60}$ in which patients with a history of myocardial infarction and symptomatic or asymptomatic ventricular ectopy ( $\geq 6$ ventricular premature depolarizations VPDs per hour) were randomized to receive flecainide, another Vaughan Williams class IC agent encainide, or placebo for VPD suppression. Patients randomized to receive therapy with flecainide or encainide had an increased risk of total mortality and an increased risk of nonfatal cardiac arrest and death from arrhythmia. The risk of proarrhythmia associated with Vaughan Williams class IC antiarrhythmic agents seems to be highest in patients with ventricular conduction delays (QRS duration $>120$ milliseconds), structural heart disease, ventricular scar tissue, or left ventricular (LV) dysfunction. ${ }^{61}$ Consequently, flecainide should be avoided in these patients.

In addition to the risk of proarrhythmia, flecainide has potent negative inotropic activity, and has been associated with worsening heart failure in patients with coronary artery disease or preexisting heart failure (New York Heart Association NYHA class II to IV and/or LV ejection fraction $<30 \%$ ). ${ }^{59}$ Therefore, flecainide is contraindicated in patients with heart failure and reduced ejection fraction. 
Intravenous flecainide is not available in the United States, but is available in other countries. In addition to the potential for ventricular proarrhythmia in patients with structural heart disease and worsening of heart failure in patients with LV dysfunction, intravenous flecainide may be associated with hypotension.

\subsubsection{Magnesium}

Magnesium administered intravenously is often referred to as a physiologic calcium channel blocker, due to its antagonism of L- and T-type calcium channels. ${ }^{62}$ Intravenous magnesium diminishes atrial automaticity ${ }^{63}$ ) and inhibits AV node conduction. ${ }^{64}$ Intravenous magnesium is well tolerated; sinus bradycardia or AV block have been reported with an incidence of approximately $3 \%{ }^{65}$ Intravenous magnesium may also cause hypotension (approximate incidence 4\%). ${ }^{65}$ Transient adverse effects including flushing, tingling, and dizziness may occur in up to $17 \%$ of patients. ${ }^{65}$

\subsubsection{Dofetilide}

Dofetilide is a Vaughan Williams class III antiarrhythmic agent that inhibits $\mathrm{I}_{\mathrm{Kr}}$ current, ${ }^{66}$ and prolongs atrial and ventricular action potential duration. ${ }^{67}$ Although dofetilide has been shown to be effective for converting nonsurgical AF to sinus rhythm ${ }^{68}$ and for maintenance of sinus rhythm in patients with nonoperative $\mathrm{AF}^{69}$ it has not been studied specifically for prevention or management of AF after noncardiac thoracic surgery. As a result of its propensity to inhibit $\mathrm{I}_{\mathrm{Kr}}$ and prolong ventricular repolarization, dofetilide may cause $\mathrm{TdP}$, with an incidence of approximately $1 \%$ in patients with normal LV function. ${ }^{67}$ However, the incidence increases to $3.3 \%$ in patients with heart failure with reduced LV ejection fraction. ${ }^{70}$ To minimize the risk of TdP, dofetilide doses must be appropriately adjusted for kidney disease. $^{2,3}$

\subsubsection{Dronedarone}

Dronedarone is a Vaughan Williams class III antiarrhythmic agent that was developed as a potentially safer congener of amiodarone. Dronedarone is similar to amiodarone in that it inhibits multiple ion currents, including fast $\mathrm{Na}^{+}$current, $\mathrm{I}_{\mathrm{Kr}}$, acetylcholine-activated $\mathrm{K}^{+}$current, and $\mathrm{L}$ type calcium current. ${ }^{71}$ Dronedarone is also a noncompetitive $\beta$-adrenergic inhibitor. Unlike amiodarone, however, which possesses 2 iodine atoms that compose $37 \%$ of its molecular weight, dronedarone's structure does not include iodine atoms. In addition, the half-life of dronedarone (13 to 31 hours) is much shorter than that of amio- darone (10 to 40 days). ${ }^{71}$ Dronedarone's primary adverse effects include gastrointestinal distress $(16 \%)$, dizziness $(9 \%)$, and bradycardia $(3 \%))^{71}$ Dronedarone was associated with an increased incidence of mortality in a randomized, doubleblind, placebo-controlled study. ${ }^{72}$ and therefore is contraindicated in patients with NYHA class III to IV heart failure, and in those patients with unstable NYHA class II heart failure.

Dronedarone has been shown to be effective for maintenance of sinus rhythm in patients with nonsurgical paroxysmal AF. Dronedarone is contraindicated in patients with permanent $\mathrm{AF}$, due to increased mortality associated with dronedarone in that patient population. ${ }^{73}$ The efficacy of dronedarone for maintenance of sinus rhythm in patients with nonsurgical AF has not been investigated.

\subsubsection{Ibutilide}

Ibutilide is a Vaughan Williams class III antiarrhythmic agent that exerts its antiarrhythmic activity via activation of slow inward sodium current $^{74}$ and inhibition of $\mathrm{I}_{\mathrm{Kr}}{ }^{75}$ Ibutilide is effective for conversion of atrial flutter and fibrillation to sinus rhythm. ${ }^{76}$ Ibutilide is not available in an oral dosage form, and therefore is not used for maintenance of sinus rhythm. Ibutilide has been shown to be effective for conversion to sinus rhythm of AF occurring after coronary artery bypass graft surgery. ${ }^{77}$ The efficacy of ibutilide for conversion to sinus rhythm of AF after noncardiac surgery has not been investigated.

The primary adverse effect associated with ibutilide is TdP, which occurs in $1 \%$ to $3 \%$ of patients. The incidence of $\mathrm{TdP}$ is 2- to 3-fold higher in patients with heart failure as a result of reduced ejection fraction, which is a known risk factor for TdP. Ibutilide may also cause nonsustained monomorphic ventricular tachycardia in up to $8 \%$ of patients.

\subsubsection{Procainamide}

Procainamide is a Vaughan Williams class IA antiarrhythmic agent that exerts its antiarrhythmic effects through inhibition of fast sodium current as well as inhibition of $\mathrm{I}_{\mathrm{Kr}}$. In addition, a primary metabolite of procainamide, $\mathrm{N}$-acetylprocainamide, inhibits $\mathrm{I}_{\mathrm{Kr}}$ current and contributes to the overall antiarrhythmic activity of procainamide. Procainamide is effective for conversion of nonoperative AF to sinus rhythm. ${ }^{78}$ The efficacy of procainamide for conversion to sinus rhythm of AF after noncardiac thoracic surgery has not been investigated. Procainamide is no longer available in an oral dosage form, and therefore is no longer indicated for maintenance of sinus rhythm in patients with nonsurgical AF. 
The primary adverse effects associated with intravenous procainamide are hypotension, QT interval prolongation and TdP, and lengthening of the QRS complex.

\subsubsection{Propafenone}

Propafenone is a Vaughan Williams class IC antiarrhythmic agent that is a potent inhibitor of sodium conductance. ${ }^{79}$ Propafenone may also inhibit the transient outward potassium current $\left(\mathrm{I}_{\mathrm{to}}\right)$ and the ultrarapid delayed rectifier potassium $\left(\mathrm{I}_{\text {kur }}\right)$ current in atrial myocytes. ${ }^{80}$ Propafenone is effective for maintenance of sinus rhythm in patients with nonoperative $\mathrm{AF}^{81}$ In addition, single-oral dose propafenone is effective for conversion of nonsurgical AF to sinus rhythm. ${ }^{82}$ The efficacy of propafenone for prophylaxis or management of AF after noncardiac thoracic surgery has not been investigated.

Oral propafenone is well tolerated overall. Adverse effects include dizziness and blurred vision. However, propafenone possesses negative inotropic activity, and is contraindicated in patients with heart failure due to reduced ejection fraction. ${ }^{2,3}$ In addition, propafenone is contraindicated in patients with coronary artery disease or a history of myocardial infarction. Although propafenone was not studied in the CAST trial, the effects of flecainide and encainide in that study are believe to be to the result of potent sodium channel inhibition, and contraindications in patients with structural heart disease have been applied to propafenone.

\subsubsection{Sotalol}

Sotalol is an adrenergic $\beta$-receptor blocking agent ${ }^{83}$ that also prolongs atrial and ventricular action potential duration via inhibition of $\mathrm{I}_{\mathrm{Kr}}{ }^{84}$ Sotalol is effective for reducing the incidence of recurrent $\mathrm{AF}$ in patients with paroxysmal $\mathrm{AF}^{85}$ and after conversion to sinus rhythm. ${ }^{86}$ Sotalol has not been shown to be effective for conversion of AF to sinus rhythm. Sotalol has been used to reduce the risk of AF after coronary artery bypass graft (CABG) surgery. ${ }^{87}$ However, the efficacy of sotalol for prophylaxis of AF after noncardiac thoracic surgery has not been investigated.

\subsubsection{Quinidine}

Quinidine is a Vaughan Williams class IA antiarrhythmic agent that inhibits sodium conduction ${ }^{88}$ as well as conductance of a variety of potassium currents, including $\mathrm{I}_{\mathrm{Kr}}, \mathrm{I}_{\mathrm{KI}}$, and $\mathrm{I}_{\mathrm{to}}{ }^{89}$ The use of oral quinidine for management of AF has largely been discontinued, because of evidence that quinidine may increase mortality. ${ }^{2,3,90}$ Quinidine may prolong the QT interval and cause TdP. The efficacy of quinidine for prevention or management of $\mathrm{AF}$ after noncardiac thoracic surgery has not been evaluated.

\subsection{Serum drug concentration monitoring}

\subsubsection{Digoxin}

Serum drug concentration monitoring may be warranted only if toxicity is of concern.

Digoxin has a narrow therapeutic index, meaning that serum concentrations required for efficacy are similar to those that may cause toxicity. When used for heart failure, the desired therapeutic range is 0.5 to $0.9 \mathrm{ng} / \mathrm{mL}^{.91}$ The optimal therapeutic range for digoxin for the management of AF has not been established. The incidence of adverse effects associated with digoxin increases with serum concentrations greater than $2 \mathrm{ng} / \mathrm{mL} .^{92}$

During the management of AF after noncardiac thoracic surgery, monitoring of serum digoxin concentrations for assessment of efficacy is not necessary, as a strong relationship between rate control efficacy and serum digoxin concentration has not been established. Determination of serum digoxin concentration may be warranted if patients exhibit symptoms of digoxin toxicity, including nausea, vomiting, anorexia, or ventricular arrhythmias. If a serum concentration is believed to be necessary, the blood sample should be obtained at least 12 hours, and preferably 24 hours, after the previous digoxin dose, as a result of the prolonged tissue distribution phase ${ }^{93(\mathrm{pp} 410-439)}$; if the blood sample is obtained less than 12 hours after the dose, the serum concentration may be falsely increased, as a result of incomplete distribution of digoxin from serum to tissue.

To reduce the risk of digoxin toxicity in patients receiving the drug for $\mathrm{AF}$ after noncardiac thoracic surgery, serum digoxin concentration monitoring may be warranted if digoxin therapy must be continued for longer than 1 week, for those patients who remain in AF after hospital discharge. For patients with normal kidney function, the half-life of digoxin is approximately 36 hours; therefore, steady state serum concentrations require approximately 1 week. Routine determination of a steady state serum digoxin concentration after 1 week of therapy is not required in all patients. However, determination of a serum digoxin concentration after 1 week of therapy may be warranted in patients with chronic kidney disease or acute kidney injury, or in patients who are treated concomitantly with a 
drug that inhibits digoxin elimination, such as amiodarone, dronedarone, propafenone, quinidine, and verapamil. ${ }^{93(\mathrm{pp} 410-439)}$

\subsubsection{Procainamide: serum drug concentration} monitoring is not warranted

The suggested therapeutic range for procainamide efficacy is 4 to $10 \mathrm{mg} / \mathrm{L} .^{.3(p p 440-462)}$ However, this therapeutic range was determined using suppression of ventricular premature depolarizations and prevention of episodes of ventricular tachycardia. Serum procainamide concentrations have not been correlated with efficacy in AF, and therefore, desired serum procainamide concentrations for efficacy in AF are unknown. Serum concentration monitoring for intravenous procainamide for management of AF after noncardiac thoracic surgery is not warranted. The risk of adverse effects associated with intravenous procainamide can be minimized by terminating the loading dose of 20 to $50 \mathrm{mg} / \mathrm{min}$ continuous infusion if hypotension occurs, QRS duration is prolonged by $50 \%$, or a cumulative intravenous dose of $17 \mathrm{mg} / \mathrm{kg}$ has been administered. ${ }^{39}$

\subsubsection{Amiodarone: serum drug concentration} monitoring is not warranted

Serum amiodarone concentration monitoring has been performed during therapy for ventricular arrhythmias. However, a relationship between serum amiodarone concentrations and efficacy for prevention or management of AF has not been established. Similarly, a relationship between serum amiodarone concentrations and most of the adverse effects of amiodarone, particularly those that occur during short-term therapy, has not been established. Therefore, monitoring of serum amiodarone concentrations during prophylaxis or management of AF after noncardiac thoracic surgery is not warranted. However, to minimize the risk of pulmonary toxicity, it is recommended to keep total cumulative intravenous amiodarone doses to less than $2150 \mathrm{mg}$.

\subsubsection{Flecainide: serum drug concentration moni-} toring is not warranted

The therapeutic range for serum flecainide concentrations is often cited as 0.3 to $2.5 \mathrm{mg} / \mathrm{L} .^{93(\mathrm{pp} 440-462)}$ However, this therapeutic range was developed using suppression of ventricular premature depolarizations as an end point, rather than efficacy for the management of AF. A relationship between serum flecainide concentrations and efficacy for prophylaxis or management of AF, particularly that occurring after noncardiac thoracic surgery, has not been established. Serum flecainide concentration monitoring for prophylaxis or treatment of $\mathrm{AF}$ after noncardiac thoracic surgery is not warranted.

\subsection{Key limitations of drugs}

\subsubsection{Pulmonary toxicity}

A primary concern regarding the administration of intravenous amiodarone following lung resection is pulmonary toxicity, specifically ARDS. This concern was prominently identified by Van Mieghem and colleagues, ${ }^{40}$ who initiated a study to determine the comparative effectiveness of amiodarone, verapamil, or placebo for prevention of AF after pulmonary resection. The study was terminated prematurely due a high incidence of ARDS in amiodarone-treated patients, specifically in patients who had undergone pneumonectomy. At the time of discontinuation of the amiodarone arm, the drug had been administered to 32 patients, of whom 21 had undergone lobectomy and 11 had undergone pneumonectomy. No patients who underwent lobectomy developed amiodarone-associated ARDS. In contrast, 3 of 11 patients $(27 \%)$ in the amiodarone group who underwent pneumonectomy developed ARDS. The investigators recommended avoiding amiodarone administration for patients undergoing pulmonary resection.

Other investigators have administered intravenous amiodarone to patients undergoing lung surgery without adverse effects. In a prospective, randomized, unblinded amiodarone prophylaxis, ${ }^{94}$ the incidence of ARDS among the 65 amiodarone-treated patients (of whom 40 underwent lobectomy, 8 underwent bilobectomy, and 17 underwent pneumonectomy) was $0 \%$. Barbetakis and colleagues ${ }^{95}$ administered intravenous amiodarone to 43 patients for treatment of $\mathrm{AF}$ after lung resection. No patients developed ARDS; 21 of these patients underwent pneumonectomy. Riber and colleagues ${ }^{96}$ conducted a randomized, prospective, double-blind, placebocontrolled study of amiodarone for prevention of AF after lung resection. Only 2 patients of the 122 who received amiodarone underwent pneumonectomy; the remainder underwent right-side lobectomy or bilobectomy. No patients in this study developed ARDS or any pulmonary toxicity.

One potential difference in patients undergoing pneumonectomy in the Van Miegham study ${ }^{40}$ compared with these more recent trials ${ }^{94-96}$ 
include the cumulative intravenous amiodarone dose administered. In the Van Mieghem study, intravenous amiodarone was administered as a bolus of $150 \mathrm{mg}$ over 2 minutes, followed by a continuous infusion of $1200 \mathrm{mg}$ over 24 hours for 3 consecutive days, for a possible cumulative intravenous amiodarone dose of 3750 $\mathrm{mg}$. The 3 patients who developed amiodaroneinduced ARDS received cumulative intravenous amiodarone doses of 2150,3750 , and $3350 \mathrm{mg}$ before discontinuation of therapy. In the more recent studies, patients received a cumulative intravenous amiodarone dose of $1050 \mathrm{mg}$, after which oral amiodarone was initiated, ${ }^{94}$ or a loading dose of $300 \mathrm{mg}$ intravenous amiodarone before switching to oral amiodarone. ${ }^{96}$ In the Barbetakis study, ${ }^{95}$ intravenous amiodarone was administered as a loading dose of $5 \mathrm{mg} / \mathrm{kg}$ over 5 minutes, followed by $15 \mathrm{mg} / \mathrm{kg}$ for an undefined time period. In addition, in the Van Miegham study, the 3 patients who developed amiodaroneassociated ARDS underwent right-sided pneumonectomy, which is associated with a higher risk of postoperative ARDS than other types of lung surgery.

Overall, administration of amiodarone at the dose shown to be effective by Riber and colelagues ${ }^{96}$ (300 mg intravenous loading dose followed by $600 \mathrm{mg}$ orally twice daily for 5 days) seems to be safe and effective for prevention of AF after pulmonary resection.

\subsubsection{QT interval prolongation/torsades de pointes}

Several of the drugs that may be used for prophylaxis or management of postoperative AF may cause QT interval prolongation, and therefore pose a risk for the life-threatening polymorphic ventricular arrhythmia known as TdP. ${ }^{97}$ Drugs that prolong the QT interval are generally those that inhibit $\mathrm{I}_{\mathrm{Kr}}$, and include amiodarone, procainamide, dofetilide, dronedarone, ibutilide, sotalol, and quinidine. A Bazett-corrected $\mathrm{QT}\left(\mathrm{QT}_{\mathrm{c}}\right)$ interval greater than $500 \mathrm{~ms}$ markedly increases the risk for drug-induced $\mathrm{TdP}^{98}$ Patients receiving a drug that prolongs the $\mathrm{QT}_{\mathrm{c}}$ interval should have the $\mathrm{QT}_{\mathrm{c}}$ interval measured from a rhythm strip or 12-lead ECG at least once daily during therapy. In addition, because the occurrence of TdP is highly dependent on the presence of other risk factors (female sex, hypokalemia, hypomagnesemia, hypocalcemia, bradycardia, heart failure, increased serum drug concentrations), ${ }^{97,98}$ modifiable risk factors should be corrected. Serum potassium, magnesium, and calcium concentrations should be maintained in the normal range. Drug interactions leading to increased concentrations of a QT intervalprolonging drug should be avoided. Doses of renally eliminated QT interval-prolonging drugs (dofetilide, procainamide, sotalol) should be appropriately adjusted for declining kidney function. In addition, concomitant therapy with other QT interval-prolonging drugs, particularly noncardiovascular QT interval-prolonging drugs (fluoroquinolone and macrolide antibiotics, azole antifungal agents, antidepressants, antipsychotics, many others) ${ }^{97}$ should be avoided or performed cautiously.

\subsubsection{Hypotension}

Several drugs administered intravenously for prophylaxis or management of postoperative AF may cause hypotension, including diltiazem, esmolol, metoprolol, procainamide, and amiodarone. Drug-associated hypotension is more likely to occur when patients are volume depleted, which is often the case after thoracic surgery. In the population with AF after coronary artery bypass graft, hypotension associated with intravenous diltiazem was more likely when the pretreatment systolic blood pressure was less than $115 \mathrm{~mm} \mathrm{Hg} .{ }^{99}$

\subsubsection{Bradycardia}

Drugs used for ventricular rate control can also result in bradycardia through inhibition of sinus node function or $\mathrm{AV}$ nodal conduction. These drugs include amiodarone, propafenone, flecainide, esmolol, metoprolol, sotalol, and diltiazem. ${ }^{97}$ The risk is higher when combinations of sinus node or AV node-inhibiting drugs are used.

\subsubsection{Exacerbation of heart failure with reduced LV ejection fraction}

Several drugs used for prophylaxis or treatment of postoperative AF possess negative inotropic activity and are contraindicated in patients with heart failure with reduced LV ejection fraction. These drugs include diltiazem, procainamide, propafenone, and flecainide.

\section{Prevention Strategies and Their Efficacy}

Recent evidence suggest that some prevention strategies (avoiding $\beta$ blockade withdrawal for those chronically on those medications, correction of serum magnesium when abnormal) may be effective for all patients for reducing the incidence of POAF. By surveying the AATS membership, we also found that many of these strategies are currently underused (Figure 1). 


\section{Recommendations}

\subsection{Recommended prevention strategies for all patients Class I}

4.1.1. Patients taking $\beta$-blockers before thoracic surgery should continue them in the postoperative period to avoid $\beta$-blockade withdrawal. ${ }^{3,38,100-104}$ (LOE A).

\section{Class IIb}

4.1.2. Intravenous magnesium supplementation may be considered to prevent postoperative $\mathrm{AF}$ when serum magnesium level is low or it is suspected that total body magnesium is depleted..$^{31,38,105}$ (LOE C).

\section{Class III}

4.1.3. Digoxin should not be used for prophylaxis

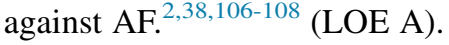

4.1.4. Catheter or surgical pulmonary vein isolation (at the time of surgery) is not recommended for prevention of POAF for patients who have no previous history of $\mathrm{AF}^{109}$ (LOE C).

4.1.5. Complete or partial pulmonary vein isolation at the time of (even bilateral) lung surgery should not be considered for prevention of POAF, as it is unlikely to be effective $\mathrm{e}^{109-111}$ (LOE B).

For those patients at increased risk for the development of POAF, preventive administration of medications (diltiazem or amiodarone) may be reasonable. However, these strategies may not be useful for all thoracic surgical patients.

\subsection{Recommended prevention strategies for intermedi- ate to high-risk patients}

\section{Class IIa}

4.2.1. It is reasonable to administer diltiazem to those patients with preserved cardiac function who are not taking $\beta$-blockers preoperatively in order to prevent $\mathrm{POAF}^{2,36,112}$ (LOE B).

4.2.2. It is reasonable to consider the postoperative administration of amiodarone to reduce the incidence of POAF for intermediate and high risk patients undergoing pulmonary resection ${ }^{2,17,94-96,113}$ (LOE A).

\section{Class IIb}

4.2.3. Postoperative administration of intravenous amiodarone may be considered to prevent POAF in patients undergoing esophagectomy ${ }^{17,96,113,114}$ (LOE B).

4.2.4. Atorvastatin may be considered to prevent POAF for statin-naive patients scheduled for intermediate- and high-risk thoracic surgical procedures $^{3,115-117}$ (LOE C).

4.3. Recommended prevention strategies for the highestrisk patients

\section{Class IIb}

4.3.1. Left atrial appendage excision may be considered at the time of extensive left lung surgery for patients with preexisting AF who are considered too high a risk for anticoagulation in the perioperative period $^{2,38,118}$ (LOE C).

\section{Reasoning}

\subsection{Prevention of postoperative AF}

AF, the most common sustained arrhythmia after pulmonary and esophageal surgery, is associated with longer intensive care unit and hospital stays, increased morbidity and mortality, and higher utilization of health care resources. ${ }^{6,26}$ POAF also represents a major potentially preventable adverse outcome. Several randomized controlled studies and meta-analyses have examined the efficacy of a variety of agents including antiarrhythmics, $\beta$-blockers, and novel agents such as magnesium and statins to prevent the development of POAF in patients undergoing thoracic surgery. However, it should be appreciated that there is a dearth of data indicating that prophylactic therapy for AF improves outcomes after thoracic surgery (eg, stroke) and reduces length of hospital stay, and many of the recommendations are extrapolated from the cardiac surgery arena.

The recommendation to avoid withdrawal of $\beta$ blockers in all patients undergoing thoracic surgery is mainly derived from the cardiac surgery literature. Nattel and colleagues ${ }^{100}$ showed that abrupt propranolol withdrawal was associated with increased sensitivity to isoproterenol, and a large meta-analysis of randomized studies confirmed that acute withdrawal of $\beta$ blockers before cardiac surgery increases the risk of developing POAF. ${ }^{103}$ There are only limited data supporting the role of prophylactic $\beta$-blockers in patients undergoing thoracic surgery. ${ }^{13,101,102}$ Although two of these randomized studies showed a reduction in POAF, there was a high incidence of hypotension and bradycardia that limited the use of $\beta$-blockers in the perioperative setting. ${ }^{119}$ There remains controversy in the recent literature as to whether to initiate perioperative $\beta$-blockers in patients who are not already taking them. At recommended doses aimed at achieving a target heart rate, $\beta$-blockers may cause significant postoperative hypotension and stroke-related mortality. ${ }^{19}$ In randomized controlled trials, diltiazem has not been associated with perioperative hypotension. The ability of diltiazem to reduce AF after thoracic surgery is moderate. ${ }^{36}$

To date, the best evidence for efficacy of AF prevention in general thoracic surgery patients has been with 


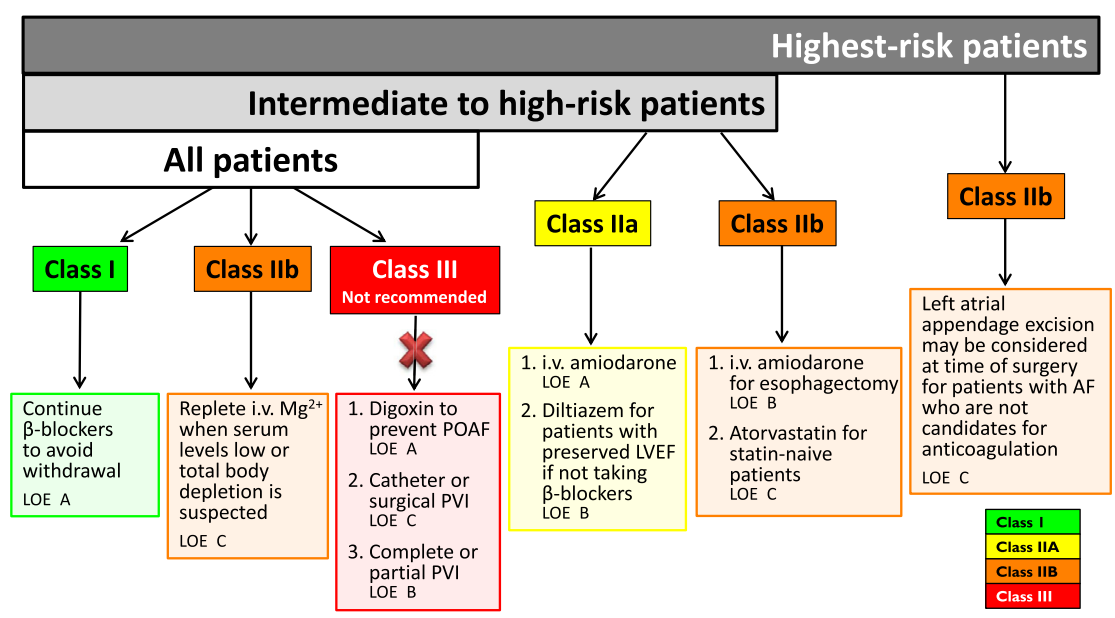

FIGURE 1. Prevention strategies and their efficacy for postoperative atrial fibrillation $(P O A F)$. $L O E$, Level of evidence; $P V I$, pulmonary vein isolation; i.v., intravenous; $L V E F$, left ventricular ejection fraction; $A F$, atrial fibrillation.

amiodarone. An important issue with any prevention efforts is the acceptance of a recommended medication by the responsible surgical team, particularly with a drug like amiodarone that has potential for side effects. The antiarrhythmic mechanism of amiodarone combines varying degrees of class III antiarrhythmic activity, $\beta$-blockade, and calcium channel antagonism. Slower postoperative heart rates with short-term use and greater than moderate efficacy in reducing $\mathrm{AF}$ may result in wider physician acceptance of amiodarone, although concerns regarding rare reports of pulmonary toxicity with right lung resection or lung transplantation may moderate its use (discussed in more detail in section 3).

\subsection{Pharmacologic therapies to prevent POAF}

\subsubsection{Amiodarone}

Efficacy of amiodarone: Tisdale and colleagues ${ }^{94}$ showed that amiodarone $1.05 \mathrm{~g}$ given by continuous intravenous infusion over the first 24 hours after pulmonary resection and then $400 \mathrm{mg}$ orally twice daily for up to 6 days, reduced the rate of POAF requiring treatment, 9 of $65(14 \%)$ in comparison with 21 of $65(32 \%)$, in an untreated control group. In a similar study, the same investigators $^{17}$ showed that continuous infusion of amiodarone $43.75 \mathrm{mg} / \mathrm{h}$ for 96 hours (total dose $4200 \mathrm{mg}$ ) was associated with a lower POAF rate of 6 of $40(15 \%)$ in patients undergoing esophagectomy compared with 16 of $40(40 \%)$ in an untreated control group. The largest trial to date by Riber and colleagues ${ }^{96}$ used a randomized, double-blind, placebo-controlled design of amiodarone given by loading $300 \mathrm{mg}$ intravenously immediately when stable after surgery followed by $600 \mathrm{mg}$ orally twice daily for up to
5 days. They showed that amiodarone-treated patients had a rate of POAF (lasting $>5 \mathrm{~min}$ ) of $9 \%$ (11 of 122), compared with placebo controls who had a rate of $32 \%$ (38 of 120). A final study of patients undergoing pulmonary resection randomized 2 groups of patients in a prospective, doubleblind design to either amiodarone (postoperative intravenous loading $5 \mathrm{mg} / \mathrm{kg}$, then $15 \mathrm{mg} / \mathrm{kg}$ for 48 hours intravenous infusion) or magnesium sulfate (preoperative loading of $80 \mathrm{mg} / \mathrm{kg}$ and then 8 $\mathrm{mg} / \mathrm{kg} / \mathrm{h}$ for 48 hours intravenous infusion after surgery). ${ }^{113}$ This study showed that the incidence of POAF (lasting $>30$ seconds) was $10 \%$ (21 of 219 ) with amiodarone and $13 \%$ (27 of 219) with magnesium. None of these studies reported any serious adverse effects caused by amiodarone except occasional bradycardia.

Safety of amiodarone: In the nonsurgical population, it is commonly accepted that amiodaronerelated pulmonary toxicity does not occur with short-term $(<1$ month) exposure. Concerns about amiodarone-related perioperative pulmonary toxicity were raised 2 decades ago in a small randomized study that was interrupted early because 3 right-sided pneumonectomy patients of a total of 11 patients who received amiodarone for prevention of POAF developed ARDS, whereas none of the 21 patients undergoing lobectomy and exposed to amiodarone developed this complication. ${ }^{40}$ The investigators acknowledged that right-sided pneumonectomy in itself was a wellestablished risk for ARDS, but nevertheless cautioned on the use of amiodarone for AF prevention after pulmonary resection. Since then, several observational and more recent prospective randomized trials failed to find a link between use of 
amiodarone for AF prevention and ARDS immediately after pulmonary resection. ${ }^{17,94,96,113,114}$ Several other studies used amiodarone for acute treatment of $\mathrm{AF}$ after general thoracic surgery, and none of these reported amiodarone-related pulmonary toxicity. Of 3 retrospective studies describing risk and treatment of AF after lung transplant, only 1 study $^{120}$ reported an association between pulmonary toxicity and amiodarone use and cautioned on the routine use of amiodarone after lung and heart-lung transplants. ${ }^{15,120}$

\subsubsection{Diltiazem}

Efficacy of diltiazem: A meta-analysis of randomized controlled trials that evaluated calcium channel blockers given immediately before, during, or after CABG surgery or valve surgery showed that these drugs reduced rates of myocardial injury and supraventricular tachycardia (SVT) ${ }^{112}$ In patients undergoing thoracic surgery, there have been 3 prospective randomized trials of nondihydropyridine calcium channel antagonists for the prevention of AF. Verapamil prophylaxis was used in a large, randomized, open-label study of patients undergoing lobectomy or pneumonectomy using somewhat aggressive loading (started within 1 hour of arrival in recovery, 10 $\mathrm{mg}$ over 10 minutes followed by $0.375 \mathrm{mg} / \mathrm{min}$ over 30 minutes) and then by continuous infusion $\left(0.125 \mathrm{mg} / \mathrm{min}\right.$ for 3 days). ${ }^{37}$ This medical regimen was associated with a nonsignificant reduction of $\mathrm{AF}$ from $15 \%$ (15 of 99 ) in placebo patients to $8 \%$ (8 of 100 ), respectively. In a small, randomized, open-label study of patients undergoing standard or intrapericardial pneumonectomy, diltiazem prophylaxis was associated with reduced overall incidence of SVT in comparison with digoxintreated patients ( 0 of 21 vs 8 of $25, P<.005$, respectively). ${ }^{108}$ In a larger follow-up, randomized, double-blind, placebo-controlled study of patients undergoing lobectomy or pneumonectomy, diltiazem started within 1 hour of arrival in recovery, $0.15 \mathrm{mg} / \mathrm{kg}$ (loading while the patient was fasting then $120 \mathrm{mg}$ orally twice a day for 14 days) reduced the rates of postoperative atrial arrhythmias in comparison with placebo ( 25 of 167 [15\%] vs 40 of 163 [25\%], respectively, $P=.03$ ). ${ }^{36}$

Safety of diltiazem: With the doses described in a randomized open-label study of verapamil given early after lobectomy or pneumonectomy, 14\% of the patients experienced hypotension and $9 \%$ had bradycardia requiring temporary interruption of the drug infusion. ${ }^{37}$ In contrast, mild transient hypotension was reported in 4\% (6 of 163) of diltiazem-treated patients, especially early after surgery, with resumption of diltiazem therapy soon thereafter. ${ }^{36}$

\subsubsection{Novel therapies to prevent postoperative AF} Inflammation and oxidative stress play an important role in the pathogenesis of AF. ${ }^{121}$ Several studies have examined the role of statin therapy in preventing POAF. One of the largest $(n=200)$ randomized studies evaluated the role of atorvastatin, given 7 days before and 7 days after cardiac surgery. Those patients who received the statin demonstrated a $22 \%$ reduction in incidence of POAF. Amar and colleagues ${ }^{115}$ conducted a prospective study of 131 patients undergoing major lung or esophageal surgery to evaluate the relationship between C-reactive protein and POAF. A secondary analysis in this study showed that in the subset of patients receiving preoperative statins, the risk of developing AF was almost 3 -fold lower than in those not taking them. Two meta-analyses of randomized studies examining prophylactic statin therapy was performed that involved more than 2200 patients. ${ }^{116-117}$ These studies supported the role of statins in preventing POAF in statin-naive patients undergoing high-risk cardiac and noncardiac surgery or after acute coronary syndromes. Additional randomized placebo-controlled studies will be required before statin therapy can be recommended as a Class I or IIa indication to prevent POAF in statin-naive patients undergoing moderate to high-risk lung surgery. As in other patients undergoing noncardiac surgery, most physicians continue statin therapy preoperatively to avoid withdrawal.

There is strong evidence supporting the use of magnesium supplementation to prevent POAF in patients undergoing cardiac surgery. ${ }^{105}$ In the only prospective, unblinded randomized, controlled trial $(\mathrm{n}=200)$ in patients undergoing thoracic surgery, Terzi and colleagues ${ }^{31}$ demonstrated that the incidence of postoperative atrial tachyarrhythmias, mainly AF, was reduced from $23 \%$ to $11 \%$ in those patients treated with an intravenous infusion of magnesium during the perioperative period.

Three large randomized clinical trials have clearly demonstrated that prophylaxis with digoxin does not prevent and may in fact increase the incidence of POAF in patients undergoing all types of thoracic surgery. ${ }^{106-108}$ Although, acute digoxin loading may be beneficial in controlling rapid ventricular rates during $\mathrm{AF}$ in patients with 
hypotension, there is no place for digoxin prophylaxis in patients undergoing thoracic surgery.

\subsubsection{Surgical prevention strategies}

Cardiac surgery patients with preexisting AF who undergo surgical pulmonary vein isolation (PVI) and receive additional biatral linear lines of block may achieve a $75 \%$ to $85 \%$ freedom from $\mathrm{AF}$ at 6 to 12 months, and the procedure adds on average an additional 9 minutes to the surgery, without perceptible safety risks, although possibly the patients have a slightly higher risk of needing a pacemaker during the early postoperative period. ${ }^{122}$ Patients who undergo the equivalent of nearcomplete PVI associated with bilateral lung transplantation have a low incidence of AF 3 to 6 months after the procedure. ${ }^{109}$ For patients with preexisting AF who are known to tolerate the arrhythmia poorly or who have an increased bleeding risk on anticoagulants, 2 questions arise: would PVI, bilateral or unilateral, help prevent perioperative $\mathrm{AF}$, and would operative left atrial appendage exclusion lower perioperative thrombotic risk?

Multiple studies ${ }^{109-111}$ have shown that the incidence of POAF is in the $20 \%$ to $40 \%$ range even after double lung transplant, confirming that this form of AF is related to inflammatory, mechanical, and autonomic factors, in addition to pulmonary vein triggers. Unilateral PVI is not likely to be any more beneficial, and has a distinct disadvantage beyond the perioperative period. $^{123}$

Excision of the left atrial appendage can be performed safely, with efficacy rates approaching $87 \%$, after a learning curve, ${ }^{118}$ but there are no studies that show a reduction in perioperative thrombotic events. The Left Atrial Appendage Occlusion Study is ongoing. Data from the Watchman $^{124}$ and Prevail trials cannot necessarily be extrapolated to operative left atrial appendage exclusion, and neither of these studies involved patients undergoing thoracotomy. Although the alteration in left atrial compliance and filling pressures after appendage exclusion may be small, the effects may be different in a lung population that may have a smaller atrial size than in a cardiac valve surgery population.

\section{Treatment Strategies for POAF and Their Efficacy}

The management of patients presenting with POAF requires different strategies depending on their hemody- namic stability. Although some interventions are likely to benefit all patients (see section 5.1), hemodynamically unstable patients will require urgent efforts for the restitution of sinus rhythm (section 5.2). However, for stable patients with POAF, the emphasis shifts to rate control strategies (see details in section 5.3). ${ }^{2}$

\section{Recommendations}

\subsection{Management strategies recommended for all pa- tients with new-onset POAF (Figure 2) \\ Class I}

5.1.1. Reduce or stop catecholaminergic inotropic agents if hemodynamics allow (LOE C).

5.1.2. Optimize fluid balance and maintain normal electrolyte levels (LOE C).

5.1.3. Evaluate the presence of and treat all possible correctable triggering factors. These may include bleeding, pulmonary embolism, pneumothorax, pericardial processes, airway issues, myocardial ischemia, or infection/sepsis (LOE C).

\section{Class IIb}

5.1.4. Cardiology consultation may be useful for those patients (LOE C) who:

5.1.4.1. Develop recurrent or refractory POAF.

5.1.4.2. Develop a hemodynamically unstable condition.

5.1.4.3. Are at high risk for stroke based on $\mathrm{CHA}_{2} \mathrm{DS}_{2}$-VASc score and will likely require longer-term anticoagulation.

5.1.4.4. Require a second-line antiarrhythmic medication for stabilization.

5.1.4.5. Also develop acute kidney injury.

\section{Reasoning}

For all patients with new-onset POAF after thoracic surgery, consideration should be given to triggering causes. Although inflammation provoked by surgical procedures, patient risk factors for $\mathrm{AF}$, and mechanical proximity of thoracic surgery procedures to cardiac structures are often sufficient to explain the occurrence of POAF, other triggers may need to be identified in patients with recurring, symptomatic, or refractory AF. These include bleeding, pulmonary embolism, pneumothorax, pericardial processes, airway issues, myocardial ischemia, or infection/sepsis. Minimization, weaning or discontinuation of catecholaminergic inotropic agents, if possible, optimization of fluid status, and correction of any electrolyte/metabolic disturbances may also facilitate restoration and maintenance of sinus rhythm.

As a general rule, although much, if not most, POAF is transient and largely limited to the postoperative period (2-6 weeks), consultation with a cardiologist or cardiac 


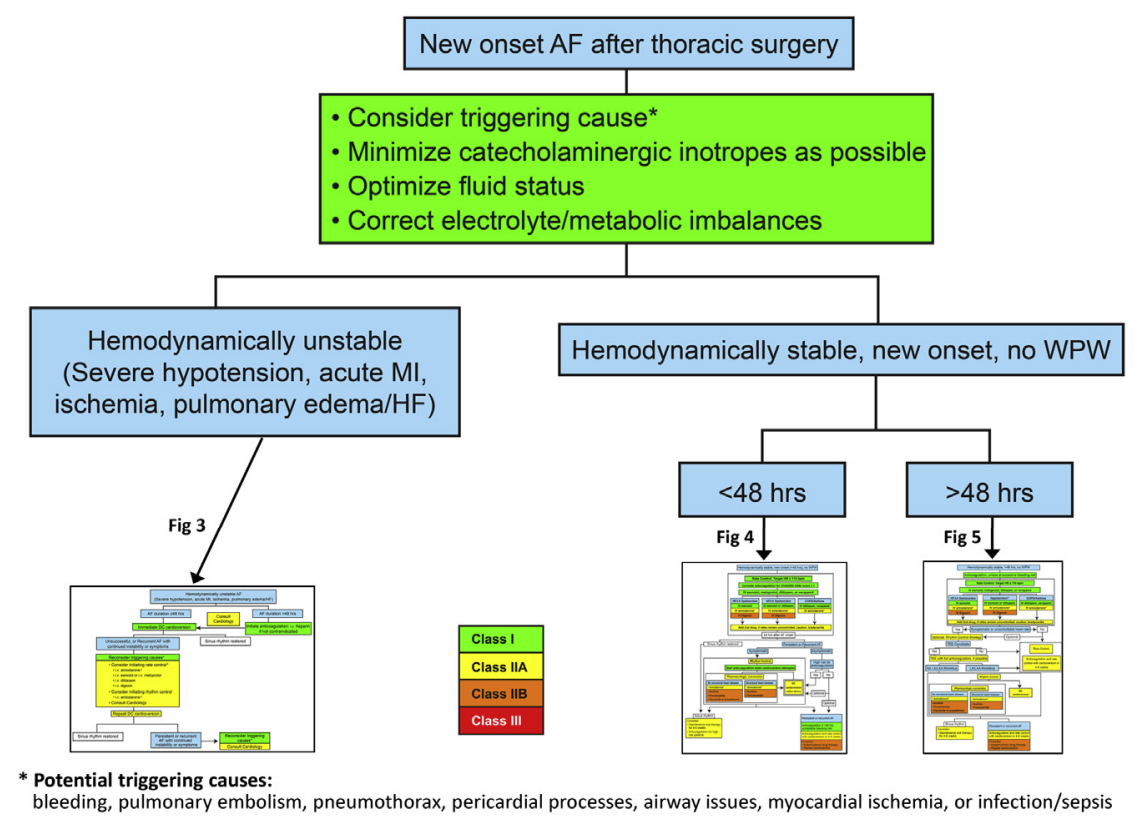

FIGURE 2. Management algorithm for postoperative atrial fibrillation (POAF). AF, Atrial fibrillation; $M I$, myocardial infarction; $H F$, heart failure; $W P W$, Wolff-Parkinson-White syndrome; $D C$, direct current; i.v./IV, intravenous; $H R$, heart rate; $L V$, left ventricular; $C O P D$, chronic obstructive pulmonary disease; $L A / L A A$, left atrial/left atrial appendage; TEE, transesophageal echocardiography.

electrophysiologist may be useful, especially for patients with recurrent or refractory POAF. This is usually for 3 issues: management of rate control during $\mathrm{AF}$; consideration of whether, when, and how to restore sinus rhythm; and consideration of anticoagulation. The first issue is usually not difficult to accomplish, and standard prophylactic use of diltiazem or $\beta$ blockers usually ensures that should POAF occur, ventricular rate control may be as simple as maintaining this therapy. However, tachybrady syndrome may complicate efforts at rate control that may require alternative medical options, rhythm control strategies, or antibradycardic pacing. The second issue may be more complex, but decisions about if, when, and how to restore sinus rhythm often benefits from direct, nuanced cardiology and/or cardiac electrophysiology involvement. Such consultation can be useful in the selection and management of antiarrhythmic medications or in determining a need for permanent pacing. The third issue is probably the most important and a challenge particularly for patients at high risk for bleeding. Cardiologists may assist with management of patients at high risk for stroke needing longer-term anticoagulation, unstable patients, or patients with acute kidney injury, which can worsen outcomes, including stroke, and limit antiarrhythmic and anticoagulant choices. Close interaction between the surgical team and the cardiology team should provide excellent, well-considered anticoagulation decisions. In the end, the patient and thoracic surgical team will be well served by a close consultative relationship with the cardiologist.

\section{Recommendations}

5.2. Recommendations for the management of the hemodynamically unstable patient with new-onset POAF (Figure 3)

\section{Class I}

5.2.1. Emergency R-wave synchronized direct current (DC) electrical cardioversion is recommended for hemodynamically unstable patients and for patients with evidence of acute myocardial ischemia or infarction. Signs of hemodynamic instability include severe symptomatic hypotension, shock, or pulmonary edema ${ }^{2-4,38}$ (LOE C).

5.2.1.1. For unstable patients with new-onset POAF of less than 48-hours duration, emergency DC cardioversion is indicated and is acceptable before initiation of anticoagulation ${ }^{2,3}$ (LOE C).

5.2.1.2. For unstable patients who undergo cardioversion more than 48 hours after the onset of $\mathrm{AF}$, and who do not have an excessive bleeding risk or other contraindication, anticoagulation should be initiated as soon as possible and continued for at least 4 weeks ${ }^{2,3}$ (LOE C).

\section{Class IIa}

5.2.2. If initial DC cardioversion is unsuccessful or hemodynamically unstable AF recurs, the following steps can be useful: 


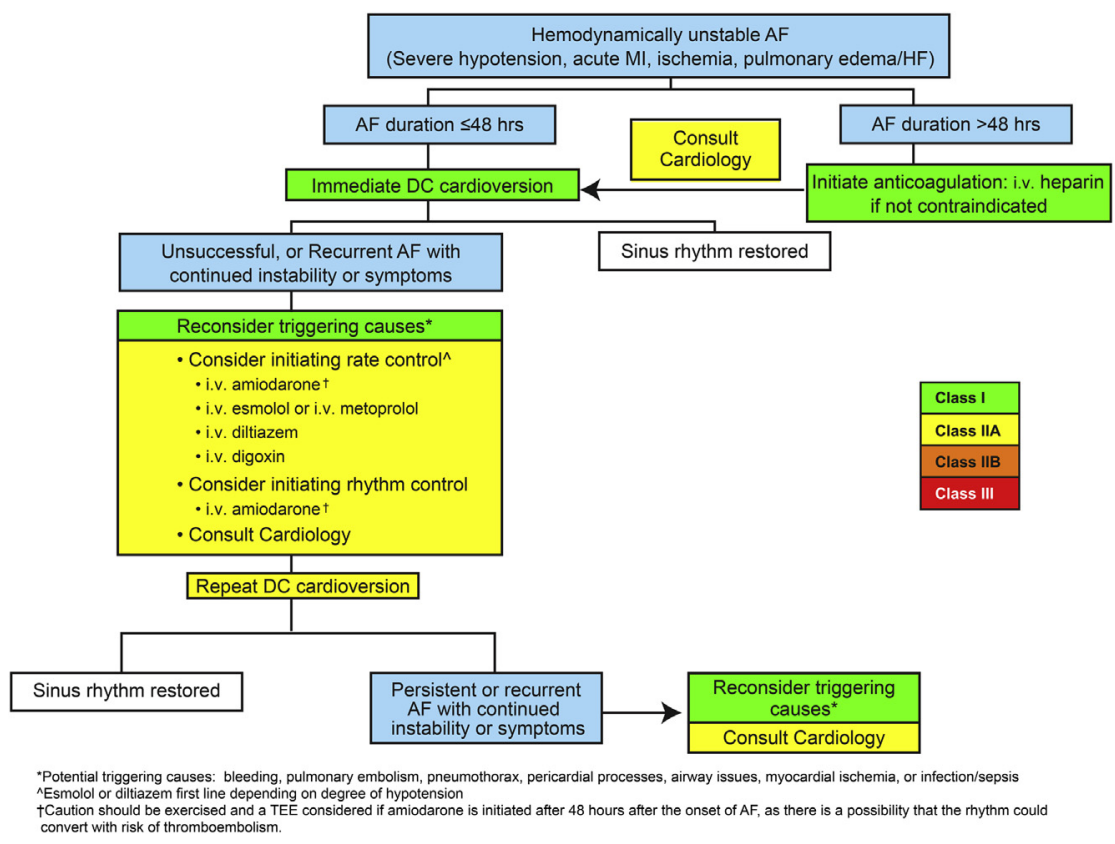

FIGURE 3. Management of the hemodynamically unstable patient with new onset postoperative atrial fibrillation (POAF). $A F$, Atrial fibrillation; $M I$, myocardial infarction; $H F$, heart failure; $D C$, direct current; i.v., intravenous; TEE, transesophageal echocardiography.

5.2.2.1. Initiate rate and possible rhythm control therapy with intravenous esmolol, diltiazem, digoxin, or amiodarone while preparing for repeat DC cardioversion (LOE C).

5.2.2.2. Repeat DC cardioversion (more likely to be successful after initiating a rhythm control agent) (LOE C).

\section{Reasoning}

Some patients with new-onset AF are hemodynamically unstable, defined as AF associated with symptomatic severe hypotension, evidence of acute myocardial ischemia or infarction, or pulmonary edema/heart failure. For such patients, immediate electrical DC cardioversion is recommended. ${ }^{2,3}$ Electrical cardioversion should be performed under deep conscious sedation with R-wave synchronized shocks. Cardioversion can be performed with biphasic or monophasic waveforms. However, biphasic waveform shocks are preferred over monophasic waveforms, as the latter can require higher defibrillation energies for success. Anterior-posterior electrode patch positioning (eg, R parasternal to L posterior or midlow sternal to posterior) may produce a more successful defibrillation vector for cardioversion of $\mathrm{AF}$ than anterior only (eg, R parasternal to anterior or anterolateral apex) positions. If DC cardioversion using 1 defibrillator patch location fails, the alternate patch position should be used.

If AF duration in the unstable patient is less than 48 hours, cardioversion can be performed before initiation of anticoagulation. ${ }^{2,3,43}$ However, for patients with AF of more than 48 hours duration who become hemodynamically unstable, there is a higher risk of left atrial or atrial appendage thrombus that could dislodge at the time of or in the days following cardioversion in the absence of anticoagulation. Thus, it is recommended that for these patients, in the absence of contraindications (such as excessive bleeding risk or known heparin sensitivity), heparin be administered concurrently with the cardioversion, and used during transition to an oral anticoagulant. The oral anticoagulant should be provided for at least 4 weeks after cardioversion, as for patients undergoing elective cardioversion ${ }^{2,3,43}$

If the initial cardioversion is unsuccessful or hemodynamically unstable AF recurs, repeat cardioversion can be attempted. To facilitate this, and while preparing for repeat cardioversion, attempts at pharmacologic rate or rhythm control may be considered with such drugs as intravenous amiodarone, esmolol, diltiazem, or digoxin. When hypotension is a problem, intravenous digoxin may be considered. However, should pharmacologic management fail, repeat electrical cardioversion is recommended.

\section{Recommendations}

5.3. Recommendations for the management of the hemodynamically stable patient with new-onset AF (Figures 4 and 5)

Primary treatment goal is rate control with rhythm control as a secondary option.

\section{Class IIa}

5.3.1. It is reasonable to manage stable, well-tolerated new-onset POAF with a rate control strategy $^{2,104,125-127}$ (LOE C). 
5.3.2. Rhythm control with antiarrhythmic drugs and/or DC cardioversion can be useful for patients with hemodynamically stable new-onset POAF who have recurrent or refractory POAF, continued symptoms, intolerance to rate control medications, or ventricular rates that cannot be adequately controlled $^{2,126,127}$ (LOE C).

5.3.3. A rhythm control approach with pharmacologic or DC cardioversion is reasonable for patients with new-onset POAF nearing 48 hours in duration, who are at high risk for bleeding, in order to avoid anticoagulation that would be otherwise indicated for AF persisting longer than 48 hours (LOE C).

\section{Reasoning}

Similar to AF occurring after cardiac surgery, new-onset POAF after thoracic surgery is often self-limited with patients returning to sinus rhythm within 4 to 6 weeks after surgery regardless of a rate or rhythm control strategy. An observational study of 30 patients with new-onset AF after lung resection and no history of heart rhythm disease reported that sinus rhythm was restored within the first 24 hours in $70 \%$ of patients treated with diltiazem, and in $67 \%$ of patients treated with amiodarone; after 48 hours, $80 \%$ in both groups were in sinus rhythm. ${ }^{125}$ AF recurred in $11(37 \%)$, but 10 converted after intravenous treatment. In a retrospective review of 41 patients who developed POAF after lung resection, $98 \%$ of AF disappeared within a day of discharge and $85.4 \%$ required pharmacologic management, but none required electrical cardioversion. ${ }^{104}$ Sinus rhythm was restored after loading with digoxin in $80 \%$, $11.5 \%$ after amiodarone, and $8.5 \%$ with both. All patients except 1 were discharged in sinus rhythm. In another study of aortic surgery in 211 patients, 22 developed POAF; 16 spontaneously converted to sinus rhythm, 2 converted chemically and 1 electrically, and 3 continued in AF at discharge, but all were in sinus rhythm documented with an ECG a mean of 14 months after discharge. ${ }^{128}$ Thus, most patients with new POAF after thoracic surgery can be expected to return to sinus rhythm regardless of a rate or rhythm control strategy.

Rate versus rhythm control strategies have been studied in randomized trials for non-POAF. ${ }^{129-133}$ The largest of these, the Atrial Fibrillation Follow-Up Investigation of Rhythm Management (AFFIRM) study, ${ }^{129}$ was powered to detect a difference in overall mortality, but showed no difference between a strategy of rhythm versus rate control in the primary end point of all-cause mortality, with a slight trend toward better survival in the rate control arm. Secondary analyses demonstrated no differences in quality of life, ${ }^{134}$ although other subanalyses demonstrated better mortality in patients in sinus rhythm or on warfarin, ${ }^{135}$ and a functional status substudy demonstrated better NYHA functional class in the rhythm control arm and longer 6-minute walk test distances in patients in sinus rhythm. ${ }^{136}$

However, there are no randomized trials studying rate versus rhythm control strategies for POAF after thoracic surgery, and there have been only small, randomized, pilot trials performed after cardiac surgery. In a randomized pilot study by Lee and colleagues ${ }^{126}$ of 50 patients with POAF after cardiac surgery, 27 were randomized to antiarrhythmic drug therapy \pm electrical cardioversion and 23 to a rate control approach. The end points were length of stay and incidence of recurrent AF. There was no significant difference in time to conversion to sinus rhythm. With multivariable Cox analyses, adjusting for other covariates, there was a trend toward a reduction in time from treatment to sinus rhythm in the antiarrhythmic arm $(P=.08)$, as well as a shorter length of stay $(P=.05)$. At termination, $91 \%$ were in sinus rhythm in the rate control arm and $96 \%$ were in sinus rhythm in the antiarrhythmic arm. Most were in sinus rhythm after 2 months. In a randomized pilot study by Soucier and colleagues ${ }^{127}$ of stable patients with new AF after cardiac surgery, 42 patients were randomized to propafenone $600 \mathrm{mg}(\mathrm{n}=20)$ versus ibutilide $1 \mathrm{mg}$ intravenously up to 2 doses $(n=10)$ versus rate control $(n=12)$. At 24 hours, $0 \%, 65 \%$, and $34 \%$ of patients in the ibutilide $(P=.01)$, propafenone $(P>.05)$, and rate control arms remained in AF. Ibutilide decreased AF duration, but recurrence rates were $90 \%, 41 \%$, and $58 \%$ in the 3 arms $(P>$ $.05)$. The 3 patients who did not convert all received propafenone. There were no differences in length of stay or rhythm at discharge. These 2 small prospective randomized pilot studies thus showed few differences between rate and rhythm control strategies.

The absence of significant differences in the small rate versus rhythm control studies of AF after cardiac surgery justifies the use of either rate or rhythm control strategies in patients with new-onset POAF who are hemodynamically stable. However, the high rate of spontaneous conversion to sinus rhythm in the first 24 hours after onset of POAF makes it reasonable to opt for an initial rate control approach in stable patients, especially over the first 24 hours. Because anticoagulation is generally recommended in patients with AF lasting longer than 48 hours, the higher risks of postoperative bleeding with anticoagulation can also justify a rhythm control approach in patients with new postoperative AF that persists longer than 24 hours despite a rate control approach. A rhythm control approach with pharmacologic or electrical cardioversion may also be reasonable in patients whose ventricular rates cannot be adequately controlled, or in patients who either do not tolerate AV nodal blockers to control ventricular rate or who remain symptomatic or hemodynamically compromised despite control of the ventricular rate.

For the patient with stable hemodynamics and minimal symptoms, a trial of rate control for the first 24 hours is 


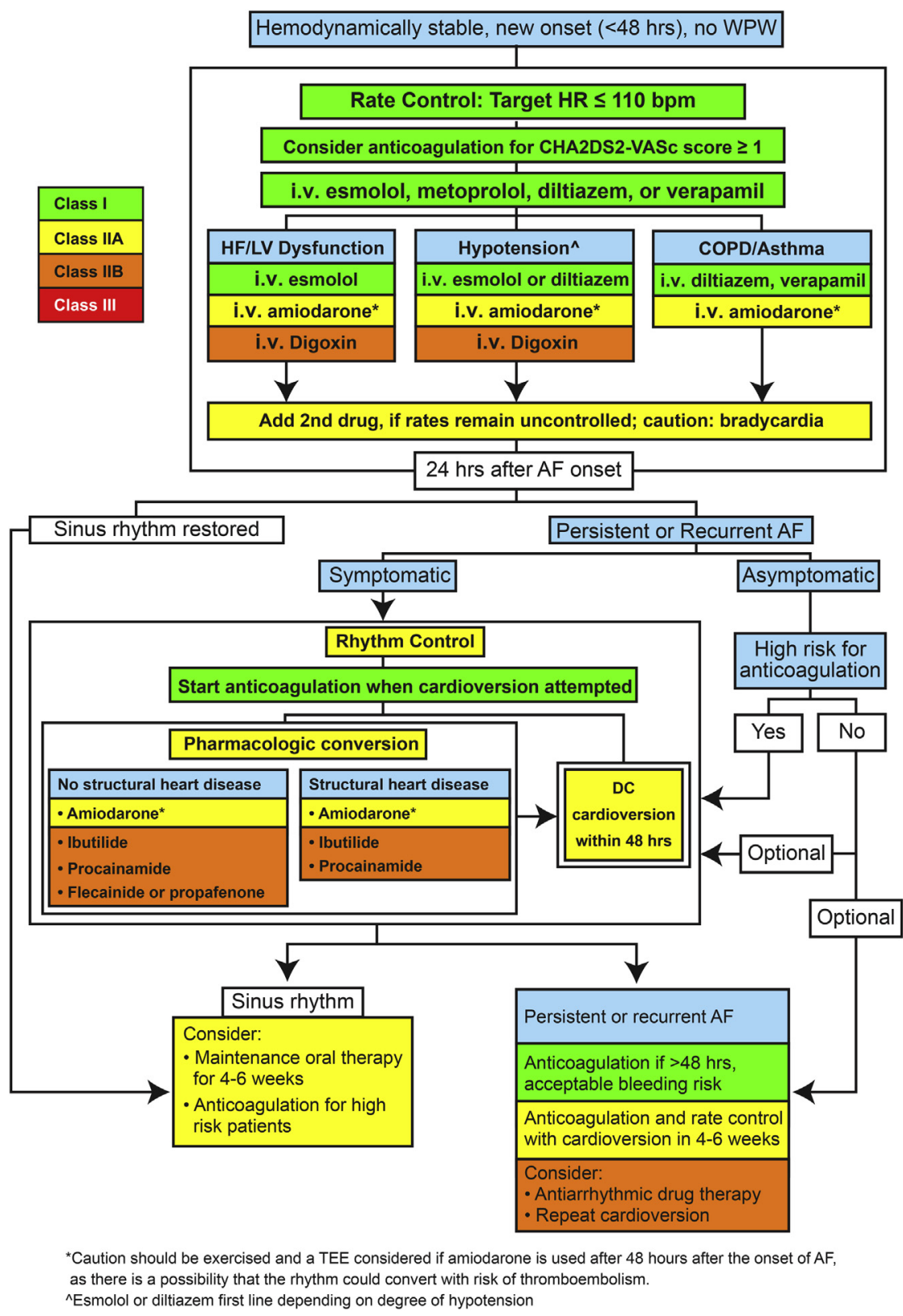

FIGURE 4. Management of the hemodynamically stable patient with new onset postoperative atrial fibrillation (POAF) of less than 48 hours duration. $W P W$, Wolff-Parkinson-White syndrome; $H R$, heart rate; i.v., intravenous; $H F$, heart failure; $L V$, left ventricular; $C O P D$, chronic obstructive pulmonary disease; $A F$, atrial fibrillation; $D C$, direct current; $T E E$, transesophageal echocardiography.

generally recommended, as a high proportion will convert to sinus rhythm within 24 hours using rate control or rhythm control agents. Inotropes should be stopped or reduced, if clinically acceptable, fluid balance optimized, and normal electrolyte balance maintained. Rate control may be achieved with intravenous esmolol or metoprolol, intravenous diltiazem, intravenous verapamil (although this carries more risk for hypotension than diltiazem), digoxin (especially if there is hypotension or heart failure), or intravenous amiodarone. If $\mathrm{AF}$ persists, $\mathrm{DC}$ cardioversion may be considered within 48 hours of onset; anticoagulation is indicated for AF persisting for more than 48 hours. Alternatively, if the AF is well tolerated, the patient could be started on anticoagulation and rate control with plans for elective cardioversion in 4 to 6 weeks. If AF is recurrent after cardioversion, antiarrhythmic therapy with repeat DC cardioversion can be continued with maintenance oral therapy for 4 to 6 weeks, or a rate control approach can be adopted with anticoagulation and plans for elective cardioversion if AF persists after 4 to 6 weeks (see Figures 2, 4, and 5). 


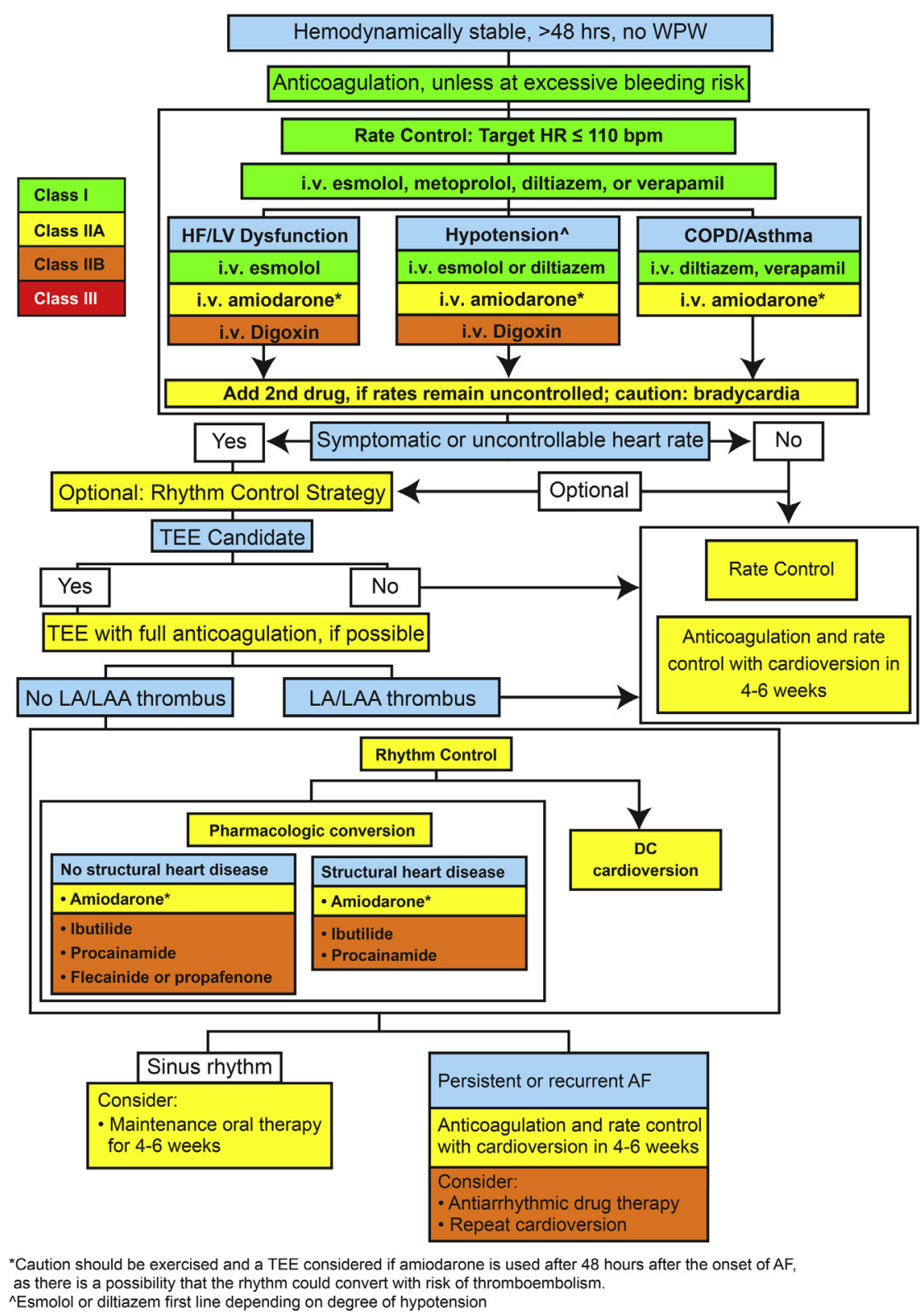

FIGURE 5. Management of the hemodynamically stable patient with new onset postoperative atrial fibrillation (POAF) of more than 48 hours duration. $W P W$, Wolff-Parkinson-White syndrome; $H R$, heart rate; i.v., intravenous; $H F$, heart failure; $L V$, left ventricular; $C O P D$, chronic obstructive pulmonary disease; TEE, transesophageal echocardiography; $L A / L A A$, left atrial/left atrial appendage; $D C$, direct current; $A F$, atrial fibrillation.

\section{Recommendations}

\subsection{Medical management of patients with new-onset POAF (Figures 4 and 5)}

\subsubsection{Rate control recommendations}

\section{Class I}

5.4.1.1. Intravenous administration of $\beta$-blockers (eg, esmolol or metoprolol) or non-dihydropyridine calcium channel blockers (diltiazem or verapamil) is recommended to achieve rate control (heart rate $\leq 110$ bpm) for patients who develop POAF with rapid ventricular response ${ }^{2,44,125}$ (LOE B).

5.4.1.1.1. Caution should be used with patients with hypotension, LV dysfunction, or heart failure ${ }^{2,38,44,125}$ (LOE B).

\section{Class IIa}

5.4.1.2. Combination use of AV nodal blocking agents, such as $\beta$-blockers (eg, esmolol or metoprolol), non-dihydropyridine calcium channel antagonists (eg, diltiazem 
or verapamil), or digoxin, can be useful to control heart rates when a single agent fails to control rates of POAF. The choice should be individualized and doses modified to avoid bradycardia ${ }^{2,38}$ (LOE B).

5.4.1.3. For patients with hypotension, heart failure, or LV dysfunction, or when other measures are unsuccessful or contraindicated, intravenous amiodarone can be useful for control of heart rate. Amiodarone could result in conversion to sinus rhythm, and if it is initiated after 48 hours of AF, both transesophageal echocardiography (TEE) when possible, to rule out left atrial (LA)/LA appendage (LAA) thrombus, and full anticoagulation should be considered $^{3,83,104,125,137}$ (LOE B).

\section{Class IIb}

5.4.1.4. For patients with heart failure, LV dysfunction, or hypotension, intravenous digoxin may be considered for rate control of POAF $^{38,99,104}$ (LOE B).

\section{Class III}

5.4.1.5. For patients with ventricular preexcitation (ie, Wolff-Parkinson-White syndrome) and POAF, use of AV nodal blocking agents, such as $\beta$-blockers (eg, esmolol or metoprolol), intravenous amiodarone, non-dihydropyridine calcium channel antagonists (eg, diltiazem or verapamil), or digoxin, should be avoided $^{2,38}$ (LOE C).

\section{Reasoning}

Achieving control of ventricular rates in AF is a first-line approach to patients with POAF after thoracic surgery. This may be achieved using intravenous or oral AV nodal blocking agents, but intravenous $\beta$-blockers or non-dihydropyridine calcium channel blockers (eg, diltiazem, verapamil) can often achieve more rapid rate control than oral agents. Choice of agents is usually based on comorbidities. $\beta$-Blockers have often been first-line therapy for ventricular rate control after cardiac surgery, and may be preferred over calcium channel blockers in patients with coronary disease. Calcium channel blockers are preferred in patients with bronchospasm limiting consideration of $\beta$-blockers, but should be avoided in patients with heart failure or severe LV dysfunction. Diltiazem is often as effective as $\beta$-blockers with less hypotension, can be titrated as a continuous infusion, and has a greater margin of safety than verapamil, which may be limited by hypotension.
The use of digoxin is generally less effective in the acute postoperative high catecholaminergic state, and it has a slower onset of action. But in the face of hypotension, digoxin may be the treatment of choice. $\beta$-Blockers and calcium channel blockers have been shown to be more effective at controlling ventricular rates with shorter times to effect than digoxin. Tisdale and colleagues ${ }^{99}$ compared intravenous diltiazem $(n=20)$ versus digoxin $(n=20)$. The end points included ventricular rate control, defined as a $20 \%$ or greater decrease in pretreatment ventricular rate and postoperative length of stay. Intravenous diltiazem achieved rate control within a mean of 10 minutes compared with 352 minutes with digoxin $(P<.0001)$. At 2 and 6 hours, successful rate control was higher in the diltiazem group, but by 24 hours there was no difference, as conversion to sinus rhythm occurred in $55 \%$ on diltiazem and $65 \%$ on digoxin. There was no difference in postoperative length of stay. However, digoxin may be particularly useful in patients with heart failure, LV dysfunction, or hypotension, or in combination with other agents. The addition of digoxin might also facilitate a lower dose of $\beta$-blockers or calcium channel blockers in patients with hypotension. Combination use of $\beta$-blockers, calcium channel blockers, or digoxin can be attempted in patients with rapid rates refractory to monotherapy, but caution should be exercised with dosage modification to avoid hypotension and bradycardia, including pauses on termination of AF.

It should be noted that in the presence of ventricular preexcitation (Wolff-Parkinson-White syndrome), AV nodal blocking agents, such as calcium channel blockers, $\beta$-blockers, digoxin, and intravenous amiodarone may potentiate rapid conduction through the accessory atrioventricular pathway due to removal of concealed conduction from the AV node. Digoxin may also shorten the AV node effective refractory period within the accessory pathway. For these patients, AV nodal blocking agents should be avoided, and antiarrhythmic medication (intravenous ibutilide, amiodarone, or procainamide) considered.

Amiodarone has also been used for ventricular rate control. However, as its antiarrhythmic properties could lead to conversion of AF to sinus rhythm, caution should be exercised if amiodarone is initiated after 24 to 48 hours after the onset of AF, as there is a possibility that the AF could convert to sinus rhythm with the attendant risk of thromboembolism. In these circumstances, TEE should be considered to exclude left atrial or left atrial appendage thrombi before initiation of amiodarone.

Parameters for optimal control of ventricular rates during AF remain controversial. The RACE II study ${ }^{44}$ evaluated a lenient (resting heart rate $<110 \mathrm{bpm}$ ) versus strict (resting heart rate $<80 \mathrm{bpm}$ ) rate control strategy in 614 patients with permanent AF. There was no difference in cardiovascular death, hospitalization for heart failure, stroke, systemic embolism, bleeding, and life-threatening 
arrhythmic events. The mean ventricular rate in the lenient control group was $85 \mathrm{bpm}$ and $76 \mathrm{bpm}$ in the strict control group at the end of the follow-up period. Although this population is different from patients with new-onset POAF, more lenient rate control (to heart rate $\leq 110$ bpm) may be preferable to strict rate control in the postoperative setting when patients are prone to hemodynamic instability or hypotension. The normal metabolic response to surgery is associated with an increase in catecholamines, often manifested in sinus tachycardia in the early perioperative period and reflected in higher ventricular rates in $\mathrm{AF}$.

There are no data to suggest efficacy for adding magnesium or potassium to facilitate conversion to sinus rhythm or to improve rate control after thoracic surgery. However, it seems reasonable to recommend maintaining normal levels.

\section{Recommendations}

\subsubsection{Recommendations for the use of antiarrhythmic drugs (Figure 6, $A$ and $B$ )}

\section{Class IIa}

5.4.2.1. Restoration of sinus rhythm with pharmacologic cardioversion is reasonable in patients with symptomatic, hemodynamically stable POAF $^{138-140}$ (LOE C).

5.4.2.1.1. Intravenous amiodarone can be useful for pharmacologic cardioversion of POAF $^{15,95,104,125,137}$ (LOE B).

5.4.2.2. It is reasonable to administer antiarrhythmic medications in an attempt to maintain sinus rhythm for patients with recurrent or refractory POAF $^{2,141}$ (LOE B).

5.4.2.2.1. Amiodarone, sotalol, flecainide, propafenone, or dofetilide can be useful to maintain sinus rhythm in patients with POAF, depending on underlying heart disease, renal status and other comorbidities (see Table $7)^{2}$ (LOE B).

\section{Class IIb}

5.4.2.3. Flecainide or propafenone may be considered for pharmacologic cardioversion of POAF and maintenance of sinus rhythm if the patient has had no previous history of myocardial infarction, coronary artery disease, impaired LV function, significant LV hypertrophy, or valvular heart disease that is considered moderate or greater. These agents may need to be combined with an $\mathrm{AV}$ nodal blocking agent $^{95,142-146}$ (LOE C).

5.4.2.4. Intravenous ibutilide or procainamide may be considered for pharmacologic conversion of POAF for patients with structural heart disease and new-onset POAF, but no hypotension or manifestations of congestive heart failure. Serum electrolytes and QTc interval must be within a normal range and patients must be closely monitored during and for at least 6 hours after the infusion if either ibutilide or procainamide ${ }^{77,143,147-149}$ (LOE B).

5.4.2.5. Intravenous ibutilide or procainamide may be considered for patients with POAF and an

\section{Class III} accessory pathway ${ }^{2,38}$ (LOE B).

5.4.2.6. Flecainide and propafenone should not be used to treat POAF in patients with a history of a previous myocardial infarction, coronary artery disease, and/or severe structural heart disease, including severe left ventricular hypertrophy, or significantly reduced left ventricular ejection fraction $^{2,60}$ (LOE B).

5.4.2.7. Dronedarone should not be used for treatment of POAF in patients with heart failure e $^{2,72}$ (LOE B).

\section{Reasoning}

For patients with symptomatic but hemodynamically stable AF after thoracic surgery, consideration should be given to restoring sinus rhythm with pharmacologic cardioversion. ${ }^{138-140}$ Although 1 study demonstrated a cardioversion rate of $86 \%$ with intravenous amiodarone in patients undergoing pulmonary resection for lung carcinoma, ${ }^{95,125}$ a meta-analysis that included both medical and POAF suggested a slightly lower rate of conversion of $76 \%$. ${ }^{15,95,104,125,137,142}$ The class IC antiarrhythmic drugs (flecainide, propafenone) may also be considered to restore and maintain sinus rhythm. Reisinger and colleagues ${ }^{143}$ compared the efficacy and safety of intravenous flecainide versus intravenous ibutilide in patients with recent-onset $\mathrm{AF}$ and showed that the rate of cardioversion was similar $(56 \%$ vs $50 \%, P>$ $.05)$. However, it should be appreciated that the intravenous form of flecainide is not available in the United States and an oral loading dose of flecainide (and propafenone) would be required to restore sinus rhythm. ${ }^{95,142-146}$ It is usually customary to combine flecainide and propafenone with $\mathrm{AV}$ nodal blocking agents to prevent 1:1 atrial flutter and rapid ventricular conduction. Ibutilide is another antiarrhythmic drug that has moderate efficacy at restoring sinus rhythm. ${ }^{143}$ However, it is only available in an intravenous form and it necessitates close monitoring of serum electrolytes and QTc. Patients must be monitored during and after intravenous ibutilide for at least 6 hours. ${ }^{2,147}$

For patients with recurrent symptomatic POAF, it is reasonable to not only restore sinus rhythm but also consider maintaining sinus rhythm with antiarrhythmic 


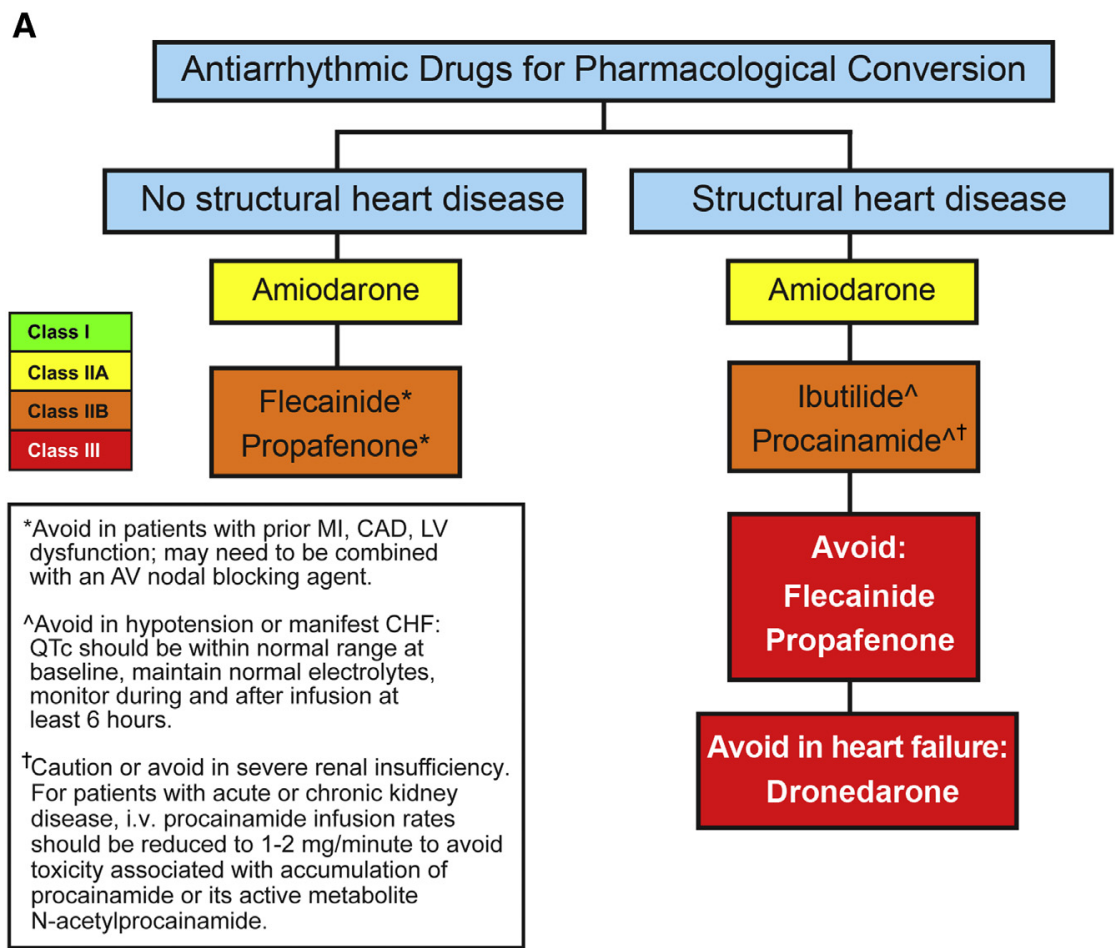

B

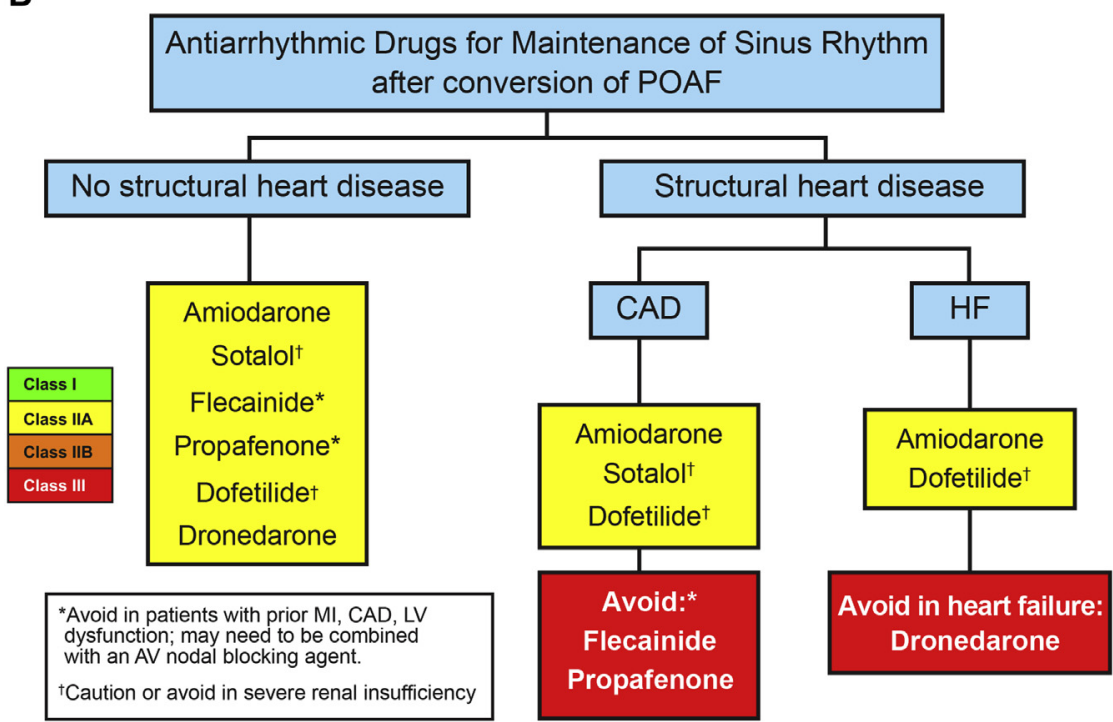

FIGURE 6. A, Antiarrhythmic drugs recommended for pharmacologic cardioversion of postoperative atrial fibrillation $(P O A F)$. B, Antiarrhythmic drugs recommended for maintenance of sinus rhythm after cardioversion of POAF. $M I$, Myocardial infarction; $C A D$, coronary artery disease; $L V$, left ventricular; $A V$, atrioventricular; $C H F$, congestive heart failure; $H F$, heart failure.

drugs. Although many membrane active drugs (amiodarone, sotalol, flecainide, propafenone, dofetilide, or dronedarone) have been shown to prevent recurrences of $\mathrm{AF}$ in both POAF and in the nonoperative setting with variable efficacy, the choice of antiarrhythmic drug is very much governed by associated comordibities, such as structural heart disease and impaired renal function. Overall, the selection of antiarrhythmic drugs to maintain sinus rhythm after thoracic surgery is similar to that outlined in the recently published $\mathrm{AF}$ guidelines for the management of nonoperative $\mathrm{AF}^{2,3}$ A review of antiarrhythmic drugs, their side effects, and interactions are outlined in section 3 and in Tables 6 and 7. 


\section{Recommendations}

5.5. Nonpharmacologic management of POAF 5.5.1. Recommendations for $D C$ cardioversion for Class I stable patients with POAF

5.5.1.1. DC cardioversion is recommended for symptomatic or relatively hemodynamically compromised patients with POAF if they do not respond promptly to pharmacologic attempts to control rapid ventricular rates $^{2,3,43}$ (LOE C).

5.5.1.2. DC cardioversion is recommended for patients without hemodynamic instability when symptoms of AF are unacceptable to the patient or when rapid ventricular rates do not respond to pharmacologic measures ${ }^{2}$ (LOE C).

\section{Class IIa}

5.5.1.3. DC cardioversion can be a reasonable alternative to pharmacologic cardiover$\operatorname{sion}^{138-140}$ (LOE C).

5.5.1.4. Pretreatment with an antiarrhythmic drug can be useful to enhance the success of DC cardioversion (as described in section 5.2.2.1.1) and to prevent recurrent $\mathrm{AF}^{2}$ (LOE B).

5.5.1.5. Caution is advised for patients with preoperative or unknown sinus node dysfunction or with patients receiving significant doses of rate controlling medications, as significant pauses can occur after DC cardioversion. For those patients, external pacing may be required and should be readily available (LOE C).

5.5.1.6. It is reasonable to repeat DC cardioversion, after administration of an antiarrhythmic medication, for patients who relapse to $\mathrm{AF}$ after successful cardiover$\operatorname{sion}^{2,43}$ (LOE C).

5.5.1.7. Patient and physician preference are reasonable considerations for selecting DC cardioversion $^{2}$ (LOE C).

5.6. Recommendations for prevention of thromboembolism for patients with stable atrial fibrillation/flutter undergoing DC cardioversion

\section{Class I}

5.6.1. For stable patients with POAF of 48 hours duration or longer, anticoagulation (with warfarin for internationalized normalized ratio [INR] 2.03.0, an NOAC or low molecular weight heparin [LMWH]) is recommended for at least 3 weeks before and 4 weeks after cardioversion, regardless of the method (electrical or pharmacologic) used to restore sinus rhythm ${ }^{2}$ (LOE B).

\section{Class IIa}

5.6.2. During the first 48 hours after the onset of POAF, the need for anticoagulation before and after DC cardioversion may be based on the patient's risk of thromboembolism $\left(\mathrm{CHA}_{2} \mathrm{DS}_{2}\right.$-VASc score; Figures 9 and 10) balanced by the risk of postoperative bleeding ${ }^{2}$ (LOE C).

5.6.3. For POAF lasting longer than 48 hours, as an alternative to 3 weeks of therapeutic anticoagulation before cardioversion of POAF, it is reasonable to perform TEE in search of thrombus in the LA or LA appendage, preferably with full anticoagulation at the time of TEE in anticipation of DC cardioversion after the $\mathrm{TEE}^{150-151}$ (LOE B).

5.6.3.1. For patients with no identifiable thrombus, DC cardioversion is reasonable immediately after the TEE examination if therapeutic anticoagulation is achieved. Anticoagulation should continue for at least 4 additional weeks although the benefits must be weighed against the risk of bleeding $^{2,151}$ (LOE C).

5.6.4. For POAF lasting longer than 48 hours in patients who are not candidates for TEE (eg, after esophageal surgery), an initial rate control strategy combined with therapeutic anticoagulation using warfarin (aiming for INR 2.0-3.0), a direct thrombin inhibitor (eg, dabigatran), factor Xa inhibitor (eg, rivaroxaban, apixaban), or LMWH is recommended for at least 3 weeks before and 4 weeks after cardioversion (LOE C).

5.6.5. Anticoagulation recommendations for cardioversion of atrial flutter are similar to those for atrial fibrillation ${ }^{2}$ (LOE C).

\section{Class III}

5.6.6. For patients with an identified thrombus, cardioversion should not be performed until a longer period of anticoagulation is achieved (usually at least 3 weeks) and in accordance with established AF guidelines ${ }^{2,150,151}$ (LOE B).

\section{Reasoning}

Electrical DC cardioversion is recommended for newonset POAF that is associated with unstable hemodynamics. DC cardioversion should be performed under deep conscious sedation with R-wave synchronized shocks. Biphasic waveform shocks are preferred over monophasic waveforms, which can require higher defibrillation energies for success.

Commonly, rate control is attempted for at least the first 24 hours, because up to $80 \%$ of patients may spontaneously 
convert with rate control alone (see rate control agents in Table 6). For patients with persistent $\mathrm{AF}$ and significant symptoms despite attempts to control ventricular response, pharmacologic or electrical cardioversion can be considered. When AF nears 48 hours in duration, such pharmacologic or electrical cardioversion may be reasonable, particularly in patients who are at high risk for bleeding, to avoid anticoagulation that would otherwise be indicated for AF persisting longer than 48 hours (see section 5.3.1). Pretreatment with an antiarrhythmic drug (see Table 7) can be useful to enhance the success of electrical cardioversion and prevent recurrent AF. However, this requires some caution if the preoperative or current status of sinus node function is unknown. If sinus node dysfunction is present, because most antiarrhythmic drugs suppress sinus node function, successful cardioversion can be associated with initial prolonged asystole and/or prolonged hypotension. In such patients, readiness for external pacing should be anticipated. If the status of sinus node function is unknown, proceeding to electrical cardioversion without administration of a precardioversion antiarrhythmic drug is reasonable.

For recurrent $\mathrm{AF}$ after initial conversion to sinus rhythm, cardioversion may be considered, often after initiation of an antiarrhythmic drug to prevent further recurrences. For recurrent or refractory $\mathrm{AF}$, evaluation for potential triggering causes should be investigated. These include bleeding, pulmonary embolism, pneumothorax, pericardial processes, airway issues, myocardial ischemia, infection, sepsis, or use of catecholaminergic inotropes. Should AF manifest as frequent paroxysms with intervening episodes of sinus rhythm, electrical cardioversion is not recommended, unless AF becomes persistent, because AF is likely to recur after cardioversion. In these situations, an antiarrhythmic drug may be beneficial.

For AF longer than 48 hours in duration, anticoagulation with warfarin (INR 2.0-3.0), a direct thrombin inhibitor (eg, dabigatran), factor Xa inhibitor (eg, rivaroxaban, apixaban), or LMWH is recommended for at least 3 weeks before and 4 weeks after cardioversion, as in other patients with AF. As an alternative to anticoagulation before cardioversion of AF, TEE may be performed in search of thrombus in the LA or LAA. It is preferable to perform the TEE on full anticoagulation with heparin or therapeutic levels of oral anticoagulants, and then to perform electrical or pharmacologic cardioversion immediately afterwards on therapeutic anticoagulation. This is preferred over performing a TEE while off anticoagulation or on subtherapeutic anticoagulation, because a thrombus may form between the time of TEE and full anticoagulation. In patients with no identifiable thrombus, cardioversion is reasonable immediately after TEE on therapeutic anticoagulation, with anticoagulation continued for at least 4 weeks afterwards, as for patients undergoing elective cardioversion. For patients with an identified thrombus, cardioversion should be deferred until a longer period of anticoagulation is achieved and in accordance with established AF guidelines. The TEEguided cardioversion approach is supported by results of the ACUTE trial, ${ }^{151}$ which enrolled 1222 patients with $\mathrm{AF}$ of greater than 2 days duration and randomized them to TEE-guided cardioversion versus warfarin anticoagulation for at least 3 weeks before cardioversion. There was no difference between the groups in the rate of embolic events, but the rate of hemorrhagic events was lower in the TEE group. Exclusion of left atrial thrombus by TEE does not preclude thromboembolism in the absence of therapeutic anticoagulation. Black and colleagues ${ }^{152}$ reported 17 patients with nonvalvular AF who had embolic events 2 hours to 7 days after cardioversion despite a TEE showing no LA thrombus. None of the patients were on therapeutic anticoagulation at the time of the embolism. Thus, the TEE-guided cardioversion strategy should be coupled with therapeutic anticoagulation at the time of and after cardioversion for patients in whom the duration of $\mathrm{AF}$ is greater than 48 hours.

It is recognized that in the thoracic surgery population, some patients will not be candidates for TEE because of esophageal procedures, including esophagectomy (or those with esophageal pathology). In these patients, if $\mathrm{AF}$ duration is greater than 48 hours, an initial rate control approach is reasonable with therapeutic anticoagulation, using warfarin (INR 2.0-3.0), a direct thrombin inhibitor (eg, dabigatran), factor $\mathrm{Xa}$ inhibitor (eg, rivaroxaban, apixaban), or LMWH, recommended for at least 3 weeks before and 4 weeks after cardioversion. Cardiac computed tomography has been used to assess for LAA thrombus, predominantly before $\mathrm{AF}$ catheter ablation. A recent meta-analysis ${ }^{153}$ reported accuracy comparable with TEE, especially when delayed images were acquired, with a sensitivity and specificity of $96 \%$ and $92 \%$ compared with TEE, a positive predictive value of $41 \%$, and a negative predictive value of $99 \%$. As clinical outcomes studies are needed to assess its clinical usefulness for cardioversion, in the case of patients with esophageal surgical procedures precluding TEE, the rate control strategy with deferred cardioversion until at least 3 weeks of therapeutic anticoagulation is achieved seems reasonable. For selected patients, cardiac computed tomography may be of some value.

\section{Recommendation}

\subsection{Recommendation for electrophysiology catheter ablation \\ Class III}

5.7.1. Catheter or surgical ablation of AF is not recommended for management of patients with postoperative AF after thoracic surgery (LOE C). 


\section{Reasoning}

Catheter ablation of $\mathrm{AF}$ is a well-established and commonly used therapeutic option for managing patients with symptomatic $\mathrm{AF}^{2,3}$ At the present time, catheter ablation of AF plays no role in the management of patients who develop $\mathrm{AF}$ in the early postoperative setting. This recommendation is based on several important considerations. First, all patients who undergo catheter ablation of AF must be anticoagulated for a minimum of 2 months after ablation. Patients who cannot be anticoagulated continuously for 2 months are not considered to be candidates for ablation. Second, catheter ablation of AF is a complex and lengthy procedure (3-6 hours) that is most commonly performed under general anesthesia. Third, it is common for AF to recur in the 2to 3-month postablation healing phase. This reflects the presence of considerable inflammation and lesion maturation that occurs after ablation. The presence of these healing phase arrhythmias means that AF ablation is an inappropriate strategy for the control of acute, symptomatic AF, such as occurs in the postoperative setting. Fourth, the efficacy of catheter ablation is modest. In optimal candidates for the procedure with paroxysmal AF who are otherwise healthy, the single procedure success rate at 12 months is $60 \%$ to $80 \%$. Late recurrences after 12 months of followup are common. Fifth, AF ablation is associated with a significant risk of complications. For more information regarding the technique, risks, indications, and outcomes of AF ablation, please refer to the 2012 Heart Rhythm Society/European Society of Cardiology/European Cardiac Arrhythmia Society Expert Consensus Statement on Catheter and Surgical Ablation of Atrial Fibrillation. ${ }^{154}$

\section{Recommendations}

\subsection{Surgical and interventional treatment options 5.8.1. Recommendations for preexisting AF Class I}

5.8.1.1. Preexisting AF should be managed according to existing guidelines for non-postoperative AF (see section 6).

\section{Reasoning}

Preexisting AF should be treated according to the existing guidelines for nonsurgical $\mathrm{AF}^{2,3}$ In the rare situation where a patient cannot be treated with anticoagulation, consideration could be given to intraoperative LAA resection or ligation. This could only be done if the patient is undergoing a left thoracotomy procedure. Regarding PVI procedures, a complete bilateral procedure can only be performed in the rare situation when bilateral thoracotomies are performed or if a clam-shell incision is used.

For new-onset AF after thoracic surgery, an intraoperative procedure is not indicated based solely on a prediction model for patients likely to develop POAF. Procedures such as PVI and/or LAA resection or ligation are not routinely practiced for the prevention of POAF in cardiac surgery, where the exposure allows such procedures to be performed easily.

It is well known that the most POAF is self-limiting in 4 to 6 weeks. For persistent AF beyond that time or POAF requiring long-term anticoagulation, patients should be referred to a cardiologist/cardiac electrophysiologist for future management according to general $\mathrm{AF}$ guidelines. If such patients are intolerant of antiarrhythmic medications, a catheter-based ablation procedure may be offered according to the existing guidelines for AF ablation. A surgical ablation procedure can be offered in the rare instance of a patient requiring a cardiac surgical procedure. A full PVI or LA maze and possible right atrial maze procedure may be performed. In addition, a LAA exclusion procedure could be also performed. If such patients are intolerant of long-term anticoagulation, LAA exclusion could be considered. ${ }^{124,155}$

\section{Management of the Patient With Preexisting AF}

Patients with preexisting AF represent a high-risk population for stroke, heart failure, and other POAFrelated complications. Some may have valvular heart disease. The management of their antiarrhythmic medications and their perioperative anticoagulation may pose a challenge (Figure 7).

\section{Recommendations}

\subsection{Criteria for obtaining cardiology consult for preop- erative $\mathbf{A F}$ \\ Class IIa}

6.1.1. Preoperative cardiology consult can be useful for patients with preoperative AF that is either newly diagnosed or persistent and symptomatic (LOE C).

6.2. Perioperative management of anticoagulation for patients on long-term (warfarin or NOAC) anticoagulation.

\section{Class I}

6.2.1. Decisions regarding the duration of interruption of anticoagulation and/or the need for perioperative heparin bridging should be based on the patient's stroke risk profile (based on their $\mathrm{CHA}_{2} \mathrm{DS}_{2}$-VASc score) (LOE C).

\section{Class IIa}

6.2.2. For patients who have a high stroke risk (based on their $\mathrm{CHA}_{2} \mathrm{DS}_{2}$-VASc score (Figures 9 and 10), history of stroke, or presence of a mechanical heart valve, perioperative bridging with a shortacting anticoagulant (ie, enoxaparin) is reasonable 


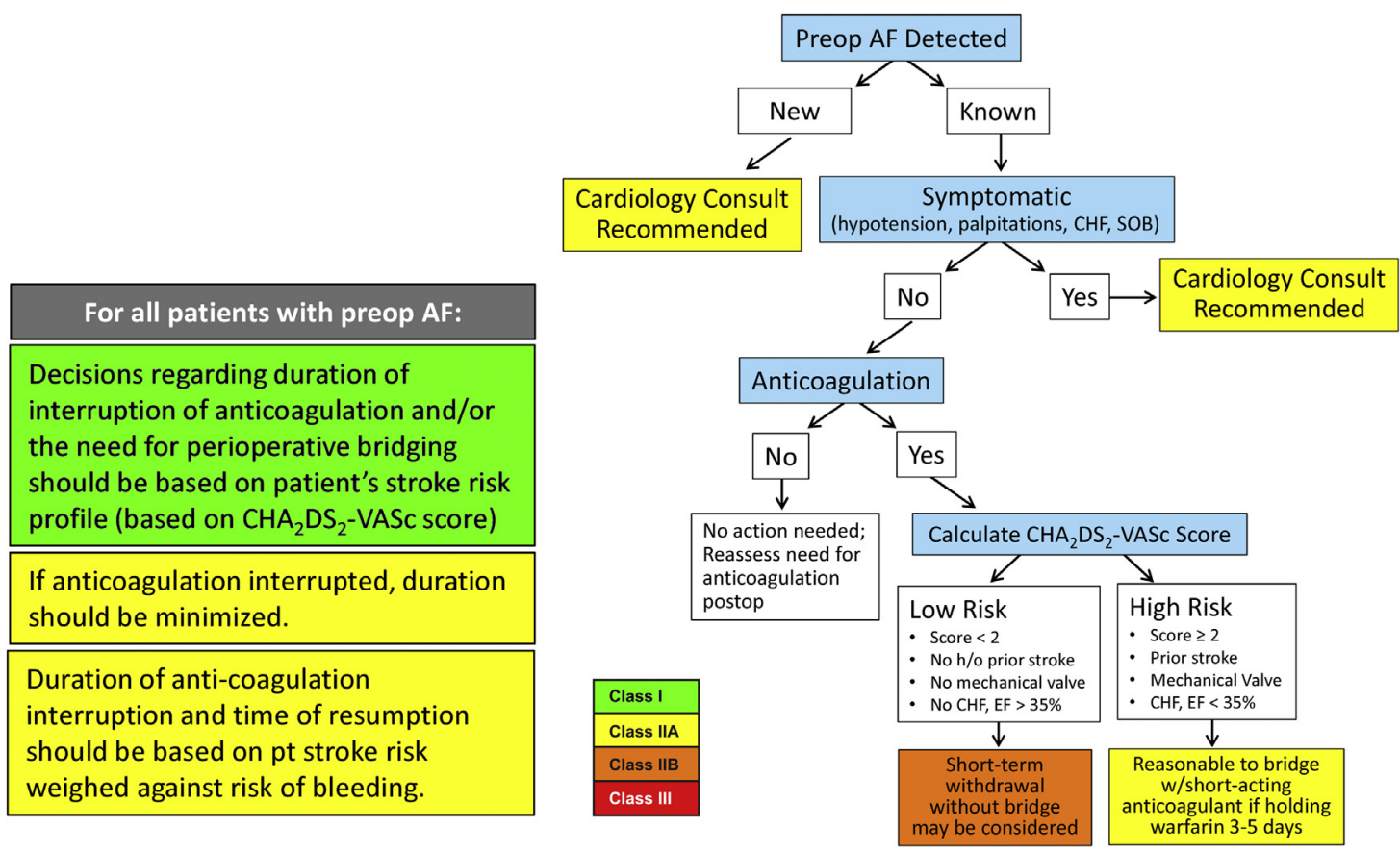

FIGURE 7. Algorithm for the management of patients with preoperative atrial fibrillation $(A F)$. Preop, Preoperative; $C H F$, congestive heart failure; $S O B$, shortness of breath; $E F$, ejection fraction.

for patients with estimated glomerular filtration rate greater than $50 \%$ when warfarin anticoagulation is withheld (LOE C).

\section{Class IIb}

6.2.3. Short-term withdrawal of anticoagulation without bridging may be considered for those patients who are on anticoagulation preoperatively as part of their treatment for persistent AF but have a $\mathrm{CHA}_{2} \mathrm{DS}_{2}$-VASc score less than 2, have not had heart failure, have an ejection fraction greater than $35 \%$, and/or for whom bridging anticoagulation would be burdensome or otherwise undesirable (LOE C).

\subsection{Postoperative resumption of anticoagulation Class IIa}

6.3.1. If anticoagulation is interrupted, the duration should be minimized. It is reasonable to base decisions about the duration of interruption and the time of resumption of anticoagulation on the patient's stroke risk profile $\left(\mathrm{CHA}_{2} \mathrm{DS}_{2}\right.$-VASc score) weighed against the risk of postoperative bleeding (LOE C).

\subsection{Postoperative follow-up Class IIb}

6.4.1. It is reasonable to consider postoperative followup with a cardiology specialist for patients with preoperatively identified $\mathrm{AF}$ who meet 1 or more of the following criteria:

6.4.1.1. Ejection fraction $45 \%$ or less or a diagnosis of systolic heart failure or cardiomyopathy

6.4.1.2. Discharged on a new rate control and/or rhythm control agent(s)

6.4.1.3. Dose of a home rhythm control agent(s) was adjusted while an inpatient

6.4.1.4. Discharged on a new anticoagulant (parenteral and/or oral) (LOE C)

\section{Reasoning}

In the Rivaroxaban Once Daily Oral direct Factor Xa Inhibition Compared with Vitamin K Antagonism for Prevention of Stroke and Embolism in Atrial Fibrillation (ROCKET AF) trial comparing warfarin and rivaroxaban for the prevention of thromboembolism in nonvalvular AF, $33 \%$ of the 14,236 patients had a temporary interruption of anticoagulation of greater than or equal to 3 days (mean duration 5 days). Eighty-one percent of these patients had persistent $\mathrm{AF}$ and more than $99 \%$ had a $\mathrm{CHADS}_{2}$ score greater than or equal to 2 (mean 3.4). ${ }^{156}$ Fifty percent of the patients had a history of stroke or transient ischemic attack and $62 \%$ had a history of congestive heart failure. Forty percent of the temporary interruptions were for procedures, although only $14 \%$ were for abdominal, thoracic, orthopedic, or cardiac procedures. The 30day stroke or systemic embolism rate of $0.36 \%$, although similar in both the rivaroxiban and warfarin groups $(0.30 \%$ vs $0.41 \%$; hazard ratio, 0.74 ; confidence interval, 
$0.36-1.5 ; P=.4$ ) was higher than the overall rate of $2.2 \% /$ year throughout the study.

In the ROCKET AF study, ${ }^{156}$ bridging of anticoagulation for temporary interruptions was tracked, and only $6 \%$ of the temporary interruptions were bridged, $98.6 \%$ with LMWH and $1.4 \%$ with fondaparinux. The bridge group was slightly older (74 vs 73 years; $P=.019$ ), had a fractionally higher $\mathrm{CHADS}_{2}$ score $(3.52$ vs 3.40 ; $P=.0094)$, more often had diabetes $(48 \%$ vs $41 \%$; $P=.0049)$, and had more temporary interruptions. The rates of previous stroke, transient ischemic attack, and congestive heart failure in the bridge group were similar to the rates in the nonbridge group. The bridged patients had only 1 stroke/embolic event, a 30-day rate of $0.17 \%$, whereas the nonbridge group 30-day event rate was $0.37 \%$. Major bleeding was similar in both groups, but the bridged group had a higher incidence of major/ nonmajor clinically relevant bleeding $(4.83 \%$ vs $3.02 \%)$. Because of the small numbers and variable reasons for bridging, statistical significance was not calculated. These data raise the concern that discontinuation of anticoagulation may expose patients to a small but significant risk of stroke.

Additional data that suggest the importance of considering the risk of stroke when contemplating the temporary interruption of anticoagulation were derived from data obtained after the closing of the ROCKET AF trial. After cessation of the study medication, the patients on rivaroxiban required an average of 13 days to achieve a therapeutic INR, whereas the patients receiving warfarin required only 3 days. The rivaroxiban group had 22 embolic events and the warfarin group had 7 events over 31 days of follow-up. ${ }^{157}$
The full role of perioperative bridging will be further elucidated in 2 ongoing randomized trials: BRIDGE (Effectiveness of Bridging Anticoagulation for Surgery; http:// clinicaltrials.gov) and PERIOP-2 (a double-blind, randomized, controlled trial of postoperative LMWH bridging therapy vs placebo bridging therapy for patients who are at high risk for arteriothromboembolism; http://clinicaltrials.gov).

\section{Management of Anticoagulation for New-Onset POAF}

In order to minimize the risk of perioperative bleeding whereas/although providing sufficient protection from the POAF-related strokes a careful evaluation of the patients' stroke risk is essential. The recently approved novel oral anticoagulants (NOAC; direct thrombin inhibitors and anti-Factor Xa agents) offer alternatives to warfarin, and are gaining popularity in the community for the long-term management of AF-related anticoagulation.

\section{Recommendations}

\section{Class I}

7.1. For the prevention of strokes for patients who develop POAF lasting longer than 48 hours, it is recommended that antithrombotic medications are administered similarly to nonsurgical patients (Figure 8). The decision to initiate therapy should be based on the benefit of reducing stroke risk versus the risk of bleeding in the postoperative period $^{33,158-160}$ (LOE A).

7.1.1. For effective anticoagulation, an INR range of 2 to 3 (with a target of 2.5) for warfarin is recommended unless otherwise contraindicated $^{161,162}$ (LOE A).

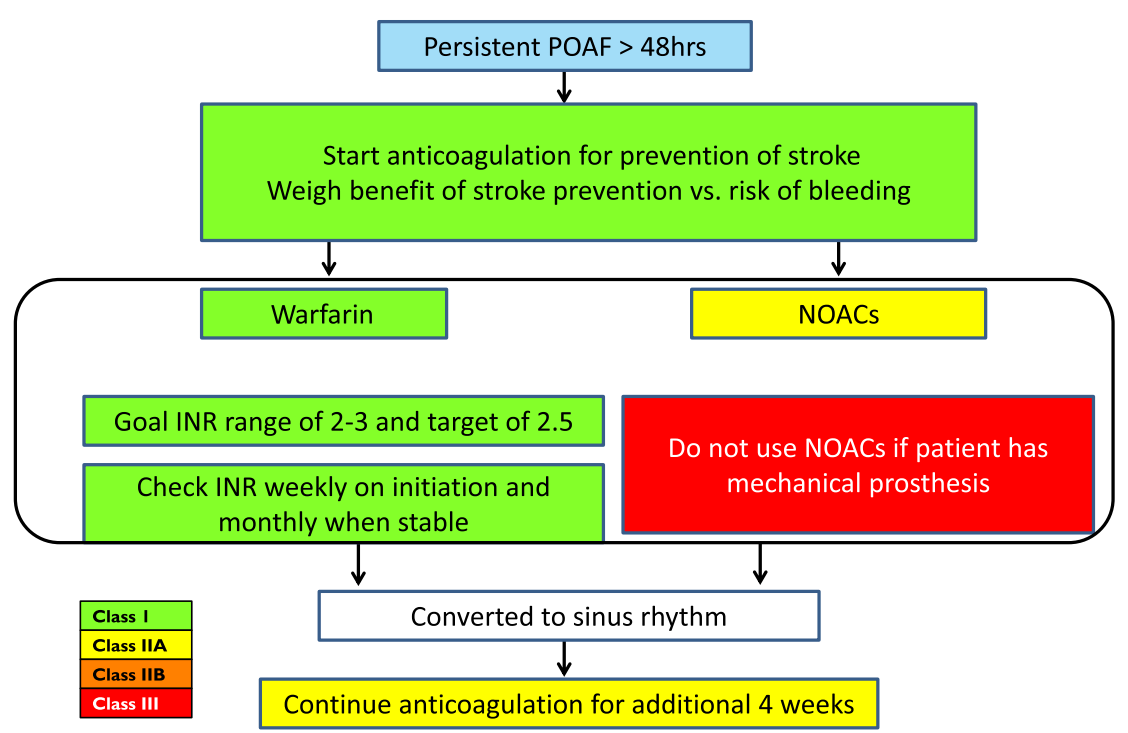

FIGURE 8. Management of anticoagulation for postoperative atrial fibrillation $(P O A F)$ lasting longer than 48 hours. NOACs, New oral anticoagulants; $I N R$, international normalized ratio. 
7.1.2. The INR should be determined at least weekly during initiation of therapy and monthly when the doses of anticoagulant and the INR are stable ${ }^{163-165}$ (LOE A).

\section{Reasoning}

The overall risk of a perioperative stroke in all patients undergoing anesthesia has been estimated at $0.5 \%$ to $0.8 \%$ in large studies of patients who have had noncardiac surgery. ${ }^{166,167}$ One of these studies use the Nationwide Inpatient Sample of 131,067 patients and determined that among 39,339 patients undergoing pulmonary lobectomy/segmentectomy, the incidence of acute ischemic stroke was $0.6 \%$, which rose to $0.8 \%$ for patients more than 65 years of age. ${ }^{166}$ Risk factors associated with perioperative stroke in that study were renal disease, atrial fibrillation, history of stroke, and cardiac valvular disease. ${ }^{166}$ Mortality in patients who developed a stroke after lung resection was 33\% compared with $3.2 \%$ in those who did not. ${ }^{166}$ The reported incidence of stroke or transient neurologic injury of $1.6 \%$ to $3.3 \%$ after cardiac operations is consistently greater for patients who develop persistent postoperative AF compared with $0.2 \%$ to $1.4 \%$ for those without $\mathrm{AF}{ }^{168}$ It has been established that oral anticoagulation with warfarin is associated with $60 \%$ to $70 \%$ reduction from the $4 \%$ to $5 \%$ overall risk of ischemic stroke per year in patients with persistent or chronic nonvalvular AF not receiving warfarin ${ }^{43,159,160,169}$ Depending of the type of surgery (total hip replacement, hemicolectomy, or lung resection), $12 \%$ to $33 \%$ of arterial thromboembolic events are fatal and more than $40 \%$ result in serious permanent disability. ${ }^{158,160}$ On the other hand, $3 \%$ of episodes of major postoperative bleeding are fatal but most patients make a full recovery. As many as $50 \%$ of bleeding episodes require a reoperation. ${ }^{158}$ A retrospective study of patients who developed new-onset AF after general thoracic surgery compared patients that received some form of antithrombotic therapy for AF with those who did not receive anticoagulation and found that the stoke rate among patients who were anticoagulated was $2.2 \%$ compared with $0.6 \%$, and these patients had a greater incidence of bleeding episodes. ${ }^{169}$ In that study, patients who were anticoagulated had more comorbidities and greater risk score for stroke. Whether individuals require short-term anticoagulation must be individualized for each patient based on the intrinsic risk for thromboembolism and the risk of bleeding ${ }^{43,159,160}$ For most types of surgery, initiation or resumption of warfarin can be undertaken 12 to 24 hours after surgery unless the patient is at special risk for bleeding, such as those with a low platelet count, prolonged excessive chest drainage, or those who might require an invasive procedure within days or weeks of discharge (eg, developing an anastomotic leak after esophagectomy). ${ }^{43,158-160,169}$ These latter patients may better be managed by LMWH and/or by a TEE-guided fast-track strategy to rule out an LAA thrombus and then receive DC cardioversion. ${ }^{159,160,168}$ Because the potential for thromboembolism with new-onset AF develops early, prompt attempts to restore sinus rhythm within this period should be made. If the arrhythmia persists beyond 24 to 48 hours, anticoagulant therapy should be considered after weighing the risk of postoperative bleeding. In a prospective study of 330 patients undergoing anatomic lung resection, 1 of 60 patients $(1.7 \%)$ with postoperative AF developed a stroke within 24 hours of onset of $\mathrm{AF}$ and Holter monitoring later showed that the initial 12 hours of AF were asymptomatic. ${ }^{36}$ Others have questioned the 48-hour window and suggest that it might be reasonable to start anticoagulation therapy in the first 48 hour if multiple risk factors for stroke are present. They further suggest that a TEE-guided strategy may prove useful in situations where both the risk of stroke and the risk of postoperative bleeding pose a dilemma regardless of the fact that $\mathrm{AF}$ was not present for 48 hours. $^{170}$

The goal of anticoagulation should balance the risk of stroke and the risk of bleeding. The range should be optimal for adequate stroke prevention but at the same time should be at the minimal bleeding threshold. In AF, an INR range of 2.0 to 3.0 with a target of 2.5 should fulfill this requirement. ${ }^{171}$ Randomized control studies have shown that warfarin therapy with an INR of 2.0 to 3.0 was associated with improved outcome compared with aspirin. ${ }^{172}$ Hylek and colleagues ${ }^{161}$ retrospectively studied 13,559 patients with nonvalvular AF and showed that an INR less than 2 at admission was associated with an increased number of strokes. Recently, in the Randomized Evaluation of Long-Term Anticoagulation Therapy (RE-LY) trial, a randomized controlled trial, compared the outcomes of warfarin versus dabigatran treatment in AF patients. Warfarin was managed with a target INR of 2.0 to 3.0 and the maximum interval between INR tests was 4 weeks. They used an algorithm to manage the INR; for example, $+15 \%$ dose/wk increase for INR less than $1.5,+10 \%$ dose/wk increase for INR from 1.5 to $1.99,-10 \%$ dose/wk decrease for INR from 3.01 to 4.00. Implementation of this algorithm resulted in an increase in time in therapeutic range (TTR). ${ }^{162}$

For in-hospital patients on warfarin, INR is measured every day until it is therapeutic. For outpatient follow-up, INR is followed every few days until it reaches the stable therapeutic target, then the interval can be prolonged as long as 4 to 6 weeks. The frequency of follow-up depends on patient compliance, drug and food interactions, interruption for surgical procedures, and existence of other comorbidities. $^{163}$ Its frequency should be increased when switching over to another type of anticoagulant, such as a 


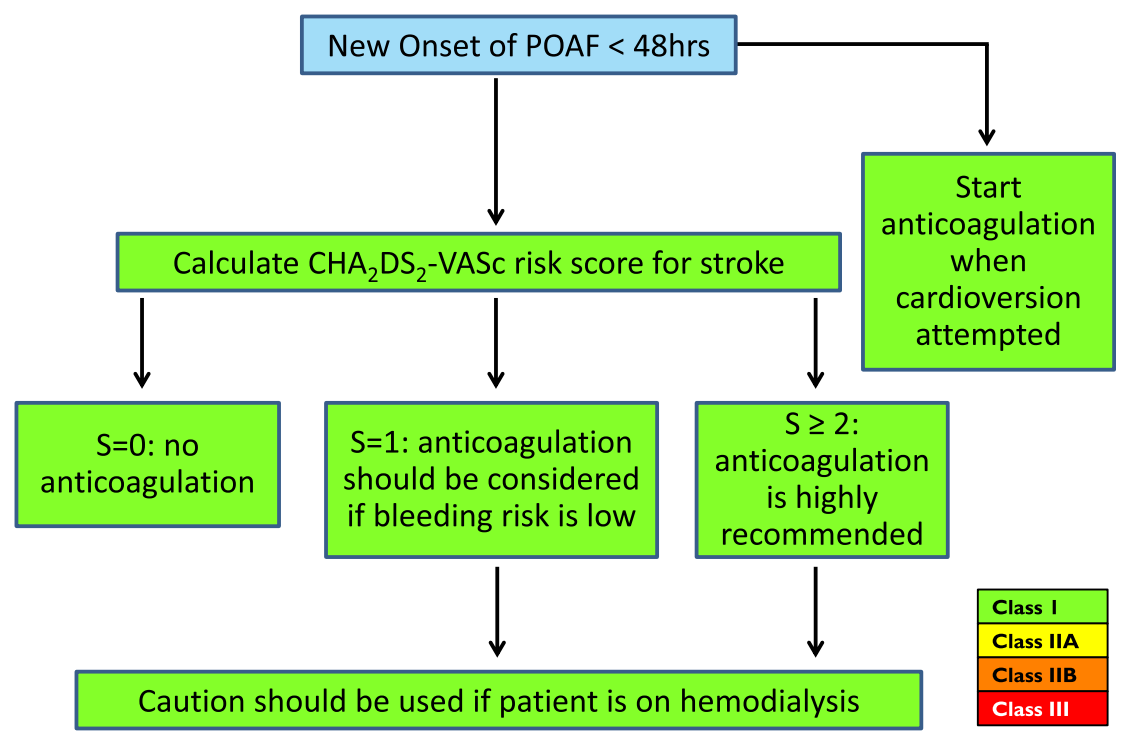

FIGURE 9. Considerations for the management of anticoagulation within the first 48 hours of postoperative atrial fibrillation $(P O A F)$.

heparin bridge. Pengo and colleagues ${ }^{164}$ randomized 124 patients to 4-week-interval and 6-week-interval follow-up of INR testing for patients with prosthetic mechanical valves and showed that there was no difference in their TTR. In a randomized study of 250 patients receiving warfarin, followed at 4-week intervals versus 12 -week intervals with phone follow-up every 4 weeks, Schulman and colleagues ${ }^{165}$ showed that the 12-week-interval group had similar TTR and bleeding/embolic events. The American College of Chest Physicians recommends a follow-up interval of up to 12 weeks if INR is stable. ${ }^{163}$

\section{Recommendations}

\section{Class I}

7.2. Anticoagulation within the first 48 hours of POAF (Figure 9) should be considered based on the $\mathrm{CHA}_{2} \mathrm{DS}_{2}$-VASc risk score (Figure 10) of the patient for stroke weighed against the risk of postoperative bleeding (LOE C).

7.2.1. For risk assessment, the following may serve as a guide: $\mathrm{CHA}_{2} \mathrm{DS}_{2}$-VASc risk score (Figure 10) for stroke $^{2,147}($ LOE A):

$\mathrm{S}=0$ : no anticoagulation recommended

$\mathrm{S}=1$ : anticoagulation should be considered if its benefits outweigh the risk of bleeding

$\mathrm{S}=2$ : anticoagulation is highly recommended if its benefits outweigh the risk of bleeding

7.2.2. The presence of impaired renal function should weigh in favor of anticoagulation. Caution should be exercised when patients on dialysis are consid- ered for anticoagulation because the benefits for those patients are less certain ${ }^{2,3,173-176}$ (LOE A).

7.2.3. If not precluded by concerns for bleeding, anticoagulation is also recommended when conversion to sinus rhythm is attempted by (DC or chemical) cardioversion (as see section 5.6) $2,3,43,159,160$ (LOE C).

\section{Reasoning}

Since the analysis by the Atrial Fibrillation Investigators (AFI) of the first 5 prospective, randomized, clinical trials comparing oral anticoagulants with placebo and sometimes with aspirin, we have learned that not all patients with atrial fibrillation have the same risk of stroke ${ }^{177,178}$ The AFI demonstrated that stroke risk may be stratified by several factors, including a previous thromboembolic event or

Components of CHA $\mathbf{D S}_{\mathbf{2}}$-VASc
\begin{tabular}{|l|c|}
\hline Risk Factor & Score \\
\hline Cardiac failure & 1 \\
\hline$\underline{\text { HTN }}$ & 1 \\
\hline Age $\geq 75$ y & 2 \\
\hline Diabetes & 1 \\
\hline Stroke & 2 \\
\hline Vascular disease (MI, PAD, & 1 \\
\hline aortic atherosclerosis) & \\
\hline Age 65-74 y & 1 \\
\hline Sex category (female) & 1 \\
\hline
\end{tabular}

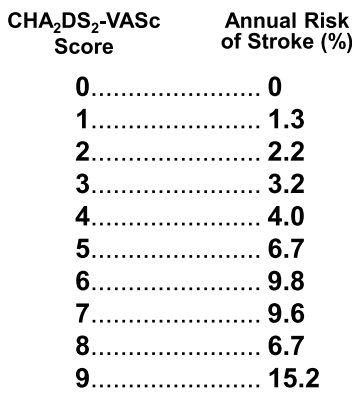

FIGURE 10. Stroke risk stratification in atrial fibrillation. From: Lip GY, Nieuwlaat R, Pisters R, Lane DA, Crijns HJ. Refining clinical risk stratification for predicting stroke and thromboembolism in atrial fibrillation using a novel risk factor-based approach: the euro heart survey on atrial fibrillation. Chest. 2010;137:263-72. HTN, Hypertension; MI, myocardial infarction; $P A D$, peripheral arterial disease. 
transient ischemic attack, hypertension, diabetes, congestive heart failure, poor left ventricular function, and age 65 years or older. Stroke risk was further stratified to mild, moderate, and severe categories. Other risks such as coronary artery disease, peripheral vascular disease, gender, thyrotoxicosis, rheumatic mitral valve disease, and hypertrophic cardiomyopathy were also important risk markers to consider.

To translate these risks derived from group data to the individual, there are now several stroke risk stratification schemes available. Initially, most guidelines adopted the $\mathrm{CHADS}_{2}$ stroke risk stratification scheme, and it has been widely used for many years. ${ }^{2,3}$ Recently, the $\mathrm{CHA}_{2} \mathrm{DS}_{2}$-VASc stroke risk stratification scheme (Figure 10) has taken prominence, having being adopted by the European Society of Cardiology, and the American College of Cardiology Foundation/American Heart Association/Heart Rhythm Society guidelines. ${ }^{2,3,147}$ A major reason for its use is that it is better at sorting out those patients with low stroke risks who do not really need anticoagulation for prophylaxis and those who do. Thus, a $\mathrm{CHADS}_{2}$ score of 0 or 1 is associated with an annual risk of $1.9 \%$ to $2.8 \%$, not really small risks at all. However, when applying the $\mathrm{CHA}_{2} \mathrm{DS}_{2}$-VASc stroke risk scheme to the same patients, the $\mathrm{CHA}_{2} \mathrm{DS}_{2}$-VASc score is anywhere from 0 to 4 . $\mathrm{A} \mathrm{CHA}_{2} \mathrm{DS}_{2}$-VASc score of 3 or 4 carries an indication for use of anticoagulation therapy, whereas a $\mathrm{CHA}_{2} \mathrm{DS}_{2}$-VASc score of 0 does not. A $\mathrm{CHA}_{2} \mathrm{DS}_{2}$-VASc score of 1 carries the recommendation to consider the use of anticoagulation therapy.

The recommendations using the $\mathrm{CHA}_{2} \mathrm{DS}_{2}$-VASc score are that if the $\mathrm{CHA}_{2} \mathrm{DS}_{2}$-VASc score is 0 , the patient does not require anticoagulation. For a $\mathrm{CHA}_{2} \mathrm{DS}_{2}$-VASc score of 1 , oral anticoagulation could be considered, and for a $\mathrm{CHA}_{2} \mathrm{DS}_{2}$-VASc of 2 or more, oral anticoagulation is generally indicated. ${ }^{147}$ Although the recently published American College of Cardiology Foundation/American Heart Association/Heart Rhythm Society AF guidelines suggested that for a CHADS-VASC score of 1, it is reasonable to consider not using antithrombotic therapy or aspirin if the burden of cardiovascular disease is otherwise low. ${ }^{2,3}$ However, most thoracic surgical patients who develop (or have) AF would likely have an indication for anticoagulation using the $\mathrm{CHA}_{2} \mathrm{DS}_{2}$-VASc risk scoring system. Because of its ease of use, and its wide acceptance, the use of the $\mathrm{CHA}_{2} \mathrm{DS}_{2}$-VASc score is recommended for the assessment of stroke risk.

Several scoring systems have been reported to identify the risk of bleeding after the initiation of anticoagulation. ${ }^{173-175}$ These scoring systems are not recommended for routine use as standard practice. However, among the risk factors, end-stage renal disease on hemodialysis is considered to pose a significant risk for bleeding when these patients are anticoagulated. In a retrospective review of 1626 dialyzed patients and nondialyzed patients, anticoagulation for dialyzed patients did not decrease the risk of stroke, but increased the incidence of bleeding episodes with $44 \%$. $^{176}$

\section{Recommendations}

\section{Class IIa}

7.3. New oral anticoagulants (dabigatran, rivaroxiban, apixiban $^{179-182}$ ) are reasonable as an alternative to warfarin (Table 8) for patients who do not have a

TABLE 8. Commonly used anticoagulants

\begin{tabular}{|c|c|c|c|c|c|c|}
\hline Drug & Mechanism & Half life (h) & Mode of clearance & Recommended doses & Significant limitations & Ref \\
\hline Warfarin & Vitamin $\mathrm{K}$ antagonist & Up to 40 & $\begin{array}{l}\text { Hepatically } \\
\text { metabolized }\end{array}$ & Variable (monitor INR) & $\begin{array}{l}\text { Multiple food and drug } \\
\text { interactions, need for } \\
\text { frequent INR monitoring } \\
\text { and dose adjustments }\end{array}$ & $2,3,147$ \\
\hline Dabigatran & Thrombin inhibitor & 13 & Renal & $\begin{array}{l}150 \mathrm{mg} \text { twice a day } 75 \mathrm{mg} \\
\text { twice a day for } \mathrm{CrCl} \\
30-50 \mathrm{~mL} / \mathrm{min}\end{array}$ & $\begin{array}{l}\text { Interaction with inhibitors of } \\
\text { P-gp, no established } \\
\text { antidote, not recommended } \\
\text { in severe renal failure }\end{array}$ & $2,3,173$ \\
\hline Rivaroxaban & Factor Xa inhibitor & $7-11$ & Renal/hepatobiliary & $\begin{array}{l}20 \mathrm{mg} \text { daily, } 15 \mathrm{mg} \text { daily for } \\
\mathrm{CrCl} 15-50 \mathrm{~mL} / \mathrm{min}\end{array}$ & $\begin{array}{l}\text { Interaction with inhibitors of } \\
\text { P-gp and CYP3A4, no } \\
\text { established antidote, not } \\
\text { recommended in severe } \\
\text { renal failure }\end{array}$ & $2,3,173$ \\
\hline Apixaban & Factor Xa inhibitor & 12.7 & Renal/fecal & $\begin{array}{l}5 \mathrm{mg} \text { twice a day } 2.5 \mathrm{mg} \text { twice } \\
\text { a day (AF) for at least } 2 \text { of } \\
\text { the following: age }>80 \mathrm{y}, \\
\text { body weight }<60 \mathrm{~kg}, \mathrm{Cr} \\
>1.5 \mathrm{mg} / \mathrm{dL}\end{array}$ & $\begin{array}{l}\text { Interaction with inhibitors of } \\
\text { P-gp and CYP3A4, no } \\
\text { established antidote, not } \\
\text { recommended in severe } \\
\text { renal failure }\end{array}$ & $2,3,173$ \\
\hline
\end{tabular}

$I N R$, International normalized ratio; $C r C l$, creatinine clearance; $P$ - $g p$, P-glycoprotein; $C Y P 3 A 4$, cytochrome P450 3A4; $A F$, atrial fibrillation; $C r$, serum creatinine. 
prosthetic heart valve, hemodynamically significant valve disease, and/or severe renal impairment or risk of gastrointestinal bleeding ${ }^{2,157,183,184}$ (LOE B).

7.4. It is reasonable to continue anticoagulation therapy for 4 weeks after the return of sinus rhythm because of the possibility of slowly resolving impairment of atrial contraction with an associated ongoing risk for thrombus formation and for delayed embolic events $^{43,159,160}$ (LOE C).

\section{Reasoning}

Newer oral anticoagulant drugs have recently become available, including dabigatran, rivaroxaban, and apixaban. Dabigatran is an oral direct thrombin inhibitor, whereas rivaroxaban and apixaban are factor $\mathrm{Xa}$ inhibitors. Compared with warfarin, these agents offer the advantage of not requiring monitoring of the INR. The efficacy of dabigatran for stroke prevention in nonsurgical, nonvalvular AF was compared with that of warfarin in the RE-LY trial, ${ }^{183}$ which was a prospective noninferiority study that randomized 18,113 patients into 3 groups: dabigatran $110 \mathrm{mg}$ twice daily or dabigatran $150 \mathrm{mg}$ twice daily, administered in blinded fashion, or warfarin titrated to an INR of 2.0 to 3.0 , administered in unblinded fashion for a median duration of 2 years. Dabigatran $150 \mathrm{mg}$ twice daily significantly reduced the risk of stroke or systemic embolism by $34 \%$ compared with warfarin. There was no significant difference in the incidence of death in either dabigatran group compared with the warfarin-treated group. There was no difference in the incidence of major bleeding between the groups on warfarin and dabigatran $150 \mathrm{mg}$ twice daily. However, there was a significantly lower incidence of hemorrhagic stroke in dabigatran 150 mg twice daily group compared with the warfarin group. The efficacy of rivaroxaban for reducing risk of stroke in patients with nonsurgical, nonvalvular AF was compared with that of warfarin in the ROCKET AF trial. $^{157}$ In this noninferiority study, 14,264 patients with AF were randomized in double-blind fashion to receive rivaroxaban $20 \mathrm{mg}$ orally daily or warfarin titrated to an INR of 2.0 to 3.0 for a median treatment period of 590 days (median follow-up, 707 days). Compared with warfarin, rivaroxaban significantly reduced the risk of stroke or systemic embolism by $21 \%$. Using an intention-to-treat analysis, there was no significant difference between rivaroxaban and warfarin in the incidence of stroke or systemic embolism. There was no difference between the groups in the incidence of major and nonmajor clinically relevant bleeding. However, the incidence of intracranial hemorrhage and fatal bleeding was significantly lower in rivaroxaban-treated patients. The efficacy of apixaban compared with that of warfarin for stroke prevention in nonsurgical, nonvalvular $\mathrm{AF}$ was investigated in the Apixaban for Prevention of Stroke in Subjects with Atrial Fibrillation (ARISTOTLE) noninferiority trial. ${ }^{184}$ Patients $(\mathrm{n}=18,201)$ with $\mathrm{AF}$ and at least 1 additional risk factor for stroke were randomized to receive apixaban $5 \mathrm{mg}$ orally twice daily or warfarin titrated to an INR of 2.0 to 3.0 for a median follow-up of 1.8 years. The risk of ischemic or hemorrhagic stroke or systemic embolism in the apixaban group was significantly lower than in the warfarin group, as was the incidence of death from any cause. The incidences of major bleeding and hemorrhagic stroke were also significantly lower in apixaban-treated patients. There was no difference between the groups in the incidence of ischemic or uncertain type of stroke.

Patients who received standard anticoagulation on discharge from the hospital can return for cardioversion between 3 and 12 weeks after initiation of anticoagulant therapy ${ }^{43,158,168}$ Patients who convert to sinus rhythm but are experiencing intermittent paroxysms of AF may be considered for anticoagulation for 1 month after the return of sinus rhythm because it has been shown that impaired atrial mechanical function can persist for several weeks after the return of sinus rhythm. ${ }^{43,159}$

\section{Recommendations}

\section{Class III}

7.5. New oral anticoagulants should be avoided for patients at risk for serious bleeding (including gastrointestinal bleeding) as they cannot be readily reversed. However, their use may be recommended in situations where achievement of a therapeutic INR with warfarin has proved to be difficult ${ }^{185}$ (LOE C).

\section{Reasoning}

A large phase 2 randomized control study, the REALIGN trial, studied patients who underwent implantation of mechanical valve (aortic or mitral) or had undergone implantation of mitral bileaflet valve less than 3 months before randomization. ${ }^{185}$ The trial was terminated because of an increase in strokes $(5 \%$ vs $0 \%$ ), myocardial infarction, and major bleeding (4\% vs $2 \%$ ) in the dabigatran group. Currently, dabigatran is contraindicated and should not be used in patients with mechanical valves.

\section{Recommendations for Long-Term Management and Follow-up of Patients With Persistent New-Onset POAF}

Those patients with POAF-related perioperative complications, and those requiring long-term management of antiarrhythmics and anticoagulants are likely to benefit from cardiology follow-up after their discharge (Figure 11). 


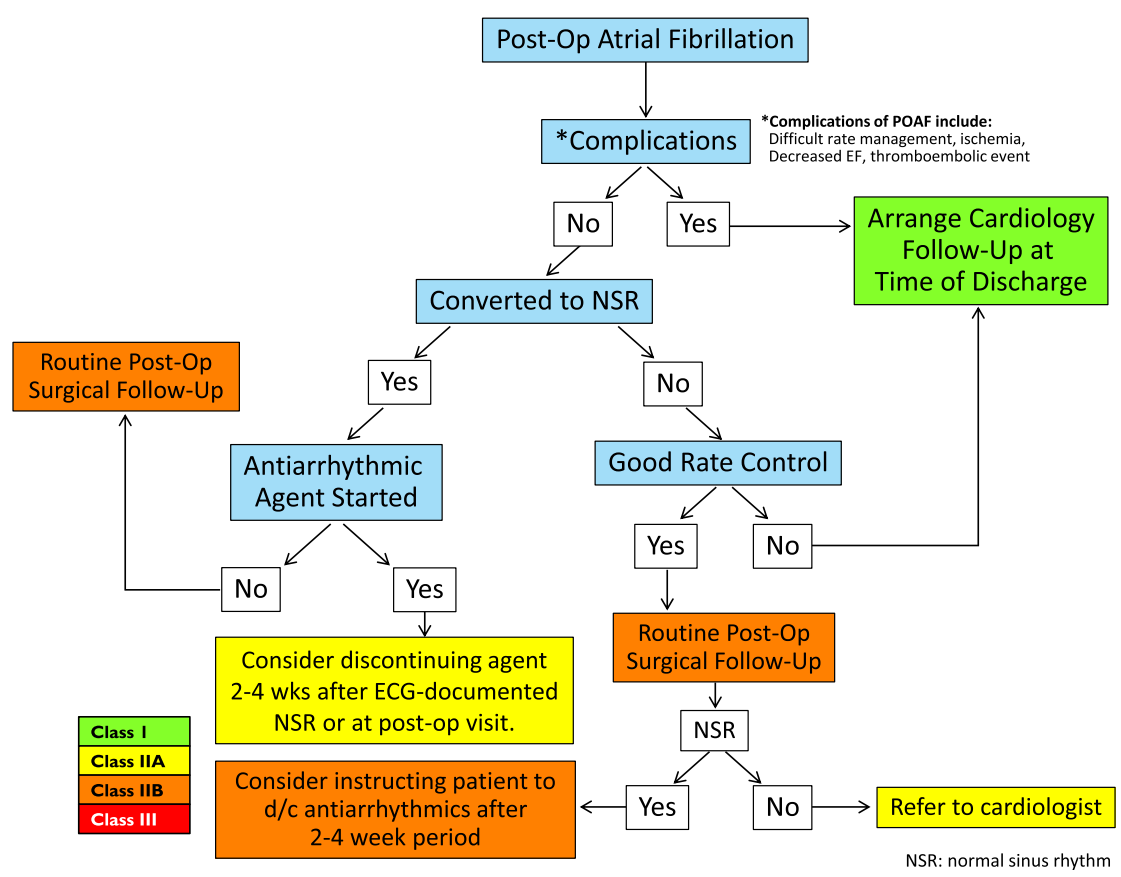

FIGURE 11. Recommendation for the postdischarge follow-up for patient with new onset postoperative atrial fibrillation (POAF). Post-op, Postoperative; $E F$, ejection fraction; NSR, normal sinus rhythm; $E C G$, electrocardiography.

\section{Recommendations}

\subsection{Postdischarge follow-up and management recom- mendations for persistent new-onset POAF. \\ Class I}

8.1.1. For patients who have a complicated in-hospital course related to their POAF, who have underlying structural heart disease, or who experience sequelae of $\mathrm{AF}$, such as myocardial infarction or decreased LVEF, follow-up with cardiology should be arranged at the time of discharge (LOE C).

\section{Class IIb}

8.1.2. Patients with well-controlled new-onset POAF (either converted to sinus rhythm or with good rate control) may be seen in routine follow-up by the surgical team without cardiology followup (LOE C).

\section{Reasoning}

Most cases of POAF are self-limiting and even when present at discharge, will have resolved by the time of followup. There is little literature regarding postdischarge risks for general thoracic patients specifically. Recommendations regarding cardiology follow-up for complicated patients seem self-evident. The appropriate timing for cardiology follow-up should be individualized before discharge. For uncomplicated patients, there is some evidence for guidance. After lung resection, Rena and colleagues ${ }^{104}$ demonstrated that $98 \%$ of POAF resolved after discharge, although this was as an older study, $80 \%$ were given digitalis. The duration was between 1 and 12 days, with an average of 2 days. It has been estimated that approximately $50 \%$ of episodes of POAF spontaneously convert to normal sinus rhythm within 12 hours. ${ }^{186}$ Given the relatively short duration of POAF in most cases, it is unclear when the first postoperative visit to the surgeon in uncomplicated cases should be. The 2010 guidelines of the Canadian Cardiovascular Society recommend that medical management of AF and anticoagulation should be reassessed at 6 to 12 weeks postoperatively, although this was intended primarily for cardiac surgical patients. This was considered a strong recommendation, with moderate evidence but no reference was given. ${ }^{187}$ Kowey and colleagues ${ }^{188}$ reported a retrospective analysis of 116 patients with POAF after coronary bypass surgery. There were 36 patients treated with antiarrhythmic and rate control drugs compared with 76 treated with rate control agents alone. Only 1 patient in each group was still in AF at 6-week follow-up. In another study, Izhar and colleagues ${ }^{189}$ randomized 129 patients after coronary bypass surgery who had converted to sinus rhythm before discharge to 1,3 , or 6 weeks of antiarrhythmic therapy. There was no difference in the rate of recurrent AF with 0,2 , and 0 patients in the 3 groups. Most of the patients were managed with amiodarone. On this basis, it seems reasonable that the timing of routine surgical follow-up should be dictated by surgical considerations, and the presence or absence of AF assessed at that time. 
Despite the relatively self-limiting nature of most cases of POAF, the long-term significance of a single episode of POAF is unknown. ${ }^{190}$ Ahlsson and colleagies ${ }^{190}$ studied the late outcome of patients who developed POAF after coronary bypass surgery and found that the development of $\mathrm{AF}$ was a risk factor for late mortality. Whether this applies to patients after noncardiac thoracic surgery is unknown. However, it seems prudent to ensure communication with the primary care physician for vigilant follow-up of cardiovascular and AF risk factors.

\section{Recommendations}

\subsection{Management of antiarrhythmic medications Class IIa}

8.2.1. For patients who have converted to sinus rhythm before hospital discharge, it is reasonable to consider discontinuation of antiarrhythmic medications 4 weeks after ECG documented return of normal sinus rhythm or at the first postoperative

\section{Class IIb} visit (usually 2-6 weeks after discharge) (LOE C).

8.2.2. For patients with new-onset POAF who were discharged in AF but who are in normal sinus rhythm (ECG confirmed) at the first postoperative visit, it may be reasonable to instruct the patients to self discontinue the antiarrhythmic medications 4 weeks after the visit if no signs of AF recur (LOE C).

\section{Reasoning}

There is no clear evidence to guide duration of antiarrhythmic therapy after noncardiac thoracic surgery. Landymore and colleagues ${ }^{191}$ followed 58 patients who underwent coronary artery bypass grafting with ambulatory Holter monitoring, including 3 patients who developed spontaneous symptomatic $\mathrm{AF}$ and received digitalis for rate control. Sixteen patients (group 2) continued taking digoxin for 8 weeks after surgery, 13 patients (group 3) discontinued digoxin treatment 5 weeks after surgery, and 14 patients (group 4) discontinued digoxin treatment 3 weeks after surgery. Twenty-four-hour Holter monitoring indicated that asymptomatic AF was common in the treatment groups after digitalization just before discharge from hospital. Recurrence of AF was rare after discharge. Yilmaz and colleagues ${ }^{192}$ performed a similar smaller study, with 120 patients who had converted to sinus rhythm (pharmacologically or with DC cardioversion) enrolled in a prospective randomized trial of placebo or 1 of 3 drugs (amiodarone, verapamil, or quinidine) postdischarge. Patients underwent 24-hour Holter monitoring 6 times over 9 months postoperatively. Recurrent AF usually developed within 15 days of discharge. AF occurred in only 1 patient $(3.33 \%)$ in group 1 , and 2 each (6.66\%) in each of the drug groups.

\section{Recommendations}

\subsection{Management of anticoagulation Class I}

8.3.1. For patients who are started on anticoagulants, the anticoagulation should continue for a minimum of 4 weeks after return to normal sinus rhythm is documented ( $\mathrm{LOE} \mathrm{C}$ ).

\section{Class IIa}

8.3.2. More prolonged anticoagulation (longer than 4 weeks after return to normal sinus rhythm) can be beneficial in the presence of stroke risk factors $\left(\mathrm{CHA}_{2} \mathrm{DS}_{2}\right.$-VASc score) or if the patient had a previous stroke. The concomitant presence of mild or moderately impaired kidney function weighs in favor of a longer period of anticoagulation (LOE B).

\subsection{Recommendations for long-term management of new-onset persistent POAF}

\section{Class IIa}

8.4.1. Patients with new-onset POAF persisting for or recurring after 4 to 6 weeks (or at the time of the first postoperative visit) can benefit from referral to a cardiologist for long-term management of stroke risk as well as antiarrhythmic or anticoagulant medications (LOE C).

\section{Reasoning}

The ideal duration of anticoagulation after POAF is unknown. European guidelines have concluded that there is insufficient evidence to make any recommendation. ${ }^{193}$ Others have concluded that it is reasonable to continue anticoagulation for 4 weeks, on the basis that atrial contraction is impaired long after the AF has ceased. ${ }^{4}$ All the current evidence in this area is level $\mathrm{C}$.

\section{RECOMMENDATIONS FOR FUTURE AATS EFFORTS}

The task force recommends the establishment of a highfidelity thoracic surgery database that uses the uniform definitions and monitoring strategies recommended here, stratifies by surgery type, and systematically documents the occurrence, duration, and complications of POAF and its treatment. The aim would be to develop risk prediction models, and eventually randomized interventional trials, for the prevention and treatment of POAF, specific to thoracic surgery. This could be most readily accomplished by enriching the STS data collection system.

\section{RECOMMENDATIONS FOR THE USE OF THE GUIDELINES}

These guidelines are best used as a guide for practice and teaching. The applicability of these recommendations to the 
individual patient should be evaluated on a case-by-case basis, and only applied when clinically appropriate. In addition, these guidelines can serve as a tool for uniform practices, to guide preoperative evaluations, and form the basis of large, multicenter cohort studies for the thoracic surgical community.

The task force received no financial support. AATS provided teleconferencing and covered the cost of a 1-day face-to-face conference for the participants. The members of this task force had no conflicts of interest related to any of the 88 recommendations made here; all their other potential conflicts of interest were disclosed in writing (Online Data Supplement 2).

A heartfelt thank you goes to Mr James M Bell for his artistic contribution in finalizing the figures and tables and to Mr Matt Eaton (AATS) for his role in coordinating all taskforce meetings and activities.

\section{References}

1. Institute of Medicine. Clinical Practice Guidelines We Can Trust. Washington, DC: National Academies Press; 2011.

2. January CT, Wann LS, Alpert JS, Calkins H, Cleveland JC, Cigarroa JE, et al. 2014 AHA/ACC/HRS guideline for the management of patients with atrial fibrillation: a report of the American College of Cardiology/American Heart Association Task Force on Practice Guidelines and the Heart Rhythm Society. Circulation. April 10, 2014 [Epub ahead of print].

3. January CT, Wann LS, Alpert JS, Calkins H, Cleveland JC, Cigarroa JE, et al. 2014 AHA/ACC/HRS guideline for the management of patients with atrial fibrillation: executive summary: a report of the American College of Cardiology/American Heart Association Task Force on Practice Guidelines and the Heart Rhythm Society. Circulation. April 10, 2014 [Epub ahead of print].

4. Fernando HC, Jaklitsch MT, Walsh GL, Tisdale JE, Bridges CD, Mitchell JD, et al. The Society of Thoracic Surgeons practice guideline on the prophylaxis and management of atrial fibrillation associated with general thoracic surgery: executive summary. Ann Thorac Surg. 2011;92:1144-52.

5. Vaporciyan AA, Correa AM, Rice DC, Roth JA, Smythe WR, Swisher SG, et al. Risk factors associated with atrial fibrillation after noncardiac thoracic surgery: analysis of 2588 patients. J Thorac Cardiovasc Surg. 2004;127:779-86.

6. Roselli EE, Murthy SC, Rice TW, Houghtaling PL. Atrial fibrillation complicating lung cancer resection. J Thorac Cardiovasc Surg. 2005;130:438-44.

7. De Decker K, Jorens PG, Van Schil P. Cardiac complications after noncardiac thoracic surgery: an evidence-based current review. Ann Thorac Surg. 2003;75: 1340-8.

8. Passman RS, Gingold DS, Amar D, Lloyd-Jones D, Bennett CL, Zhang H, et al. Prediction rule for atrial fibrillation after major noncardiac thoracic surgery. Ann Thorac Surg. 2005;79:1698-703.

9. Park BJ, Zhang H, Rusch VW, Amar D. Video-assisted thoracic surgery does not reduce the incidence of postoperative atrial fibrillation after pulmonary lobectomy. J Thorac Cardiovasc Surg. 2007;133:775-9.

10. Onaitis M, D'Amico T, Zhao Y, O'Brien S, Harpole D. Risk factors for atrial fibrillation after lung cancer surgery: analysis of the Society of Thoracic Surgeons general thoracic surgery database. Ann Thorac Surg. 2010;90:368-74.

11. Ciszewski P, Tyczka J, Nadolski J, Roszak M, Dyszkiewicz W. Lower preoperative fluctuation of heart rate variability is an independent risk factor for postoperative atrial fibrillation in patients undergoing major pulmonary resection. Interact Cardiovasc Thorac Surg. 2013;17680-6.

12. Krowka MJ, Pairolero PC, Trastek VF, Payne WS, Bernatz PE. Cardiac dysrhythmia following pneumonectomy. Clinical correlates and prognostic significance. Chest. 1987:91:490-5.

13. Hardy J. Risk factors for atrial fibrillation following extrapleural pneumonectomy, the effect of prophylactic beta blockade. Am J Respir Crit Care Med. 2012;185:A5831.

14. Lee G, Wu H, Kalman JM, Esmore D, Williams T, Snell G, et al. Atrial fibrillation following lung transplantation: double but not single lung transplant is associated with long-term freedom from paroxysmal atrial fibrillation. Eur Heart J. 2010;31:2774-82.

15. Henri C, Giraldeau G, Dorais M, Cloutier A-S, Girard F, Noiseux N, et al. Atria fibrillation after pulmonary transplantation: incidence, impact on mortality, treatment effectiveness, and risk factors. Circ Arrhythm Electrophysiol. 2012; 5:61-7.

16. Nielsen TD, Bahnson T, Davis RD. Atrial fibrillation after pulmonary transplant. Chest. 2004:126:496-500.

17. Tisdale JE, Wroblewski HA, Wall DS, Rieger KM, Hammoud ZT, Young JV, et al. A randomized, controlled study of amiodarone for prevention of atrial fibrillation after transthoracic esophagectomy. J Thorac Cardiovasc Surg. 2010;140:45-51

18. Rao VP, Addae-Boateng E, Barua A, Martin-Ucar AE, Duffy JP. Age and neoadjuvant chemotherapy increase the risk of atrial fibrillation following oesophagectomy. Eur J Cardiothorac Surg. 2012;42:438-43.

19. Ivanovic J, Maziak DE, Ramzan S, McGuire AL, Villeneuve PJ, Gilbert S, et al Incidence, severity and perioperative risk factors for atrial fibrillation following pulmonary resection. Interact Cardiovasc Thorac Surg. 2014;18:340-6.

20. Polanczyk CA, Goldman L. Supraventricular arrhythmia in patients having noncardiac surgery: clinical correlates and effect on length of stay. Ann Intern Med. 1998;129:279-85.

21. Biancari F, Mahar MAA. Meta-analysis of randomized trials on the efficacy of posterior pericardiotomy in preventing atrial fibrillation after coronary artery bypass surgery. J Thorac Cardiovasc Surg. 2010;139:1158-61.

22. The AFFIRM Investigators. Relationships between sinus rhythm, treatment, and survival in the Atrial Fibrillation Follow-Up Investigation of Rhythm Management (AFFIRM) study. Circulation. 2004;109:1509-13.

23. Bhave PD, Goldman LE, Vittinghoff E, Maselli J, Auerbach A. Incidence, predictors, and outcomes associated with postoperative atrial fibrillation after major noncardiac surgery. Am Heart J. 2012;164:918-24.

24. Imperatori A, Mariscalco G, Riganti G, Rotolo N, Conti V, Dominioni L. Atria fibrillation after pulmonary lobectomy for lung cancer affects long-term survival in a prospective single-center study. J Cardiothorac Surg. 2012;7:4.

25. Cardinale D, Martinoni A, Cipolla CM, Civelli M. Atrial fibrillation after operation for lung cancer: clinical and prognostic significance. Ann Thorac Surg. 1999;68:1827-31.

26. Irshad K, Feldman LS, Chu VF, Dorval J-F, Baslaim G, Morin JE. Causes of increased length of hospitalization on a general thoracic surgery service: a prospective observational study. Can J Surg. 2002;45:264-8.

27. Akoum N, Daccarett M, McGann C, Segerson N, Vergara G, Kuppahally S, et al. Atrial fibrosis helps select the appropriate patient and strategy in catheter ablation of atrial fibrillation: a DE-MRI guided approach. J Cardiovasc Electrophysiol. 2011;22:16-22.

28. Haïssaguerre M, Jaïs P, Shah DC, Takahashi A, Hocini M, Quiniou G, et al Spontaneous initiation of atrial fibrillation by ectopic beats originating in the pulmonary veins. N Engl J Med. 1998;339:659-66.

29. Amar D, Roistacher N, Burt M, Reinsel RA, Ginsberg RJ, Wilson RS. Clinical and echocardiographic correlates of symptomatic tachydysrhythmias after noncardiac thoracic surgery. Chest. 1995;108:349-54.

30. Tisdale JE, Wroblewski HA, Kesler KA. Prophylaxis of atrial fibrillation after noncardiac thoracic surgery. Semin Thorac Cardiovasc Surg. 2010;22:310-20.

31. Terzi A, Furlan G, Chiavacci P, Dal Corso B, Luzzani A, Dalla Volta S. Prevention of atrial tachyarrhythmias after non-cardiac thoracic surgery by infusion of magnesium sulfate. Thorac Cardiovasc Surg. 1996;44:300-3.

32. Schotten U, Verheule S, Kirchhof P, Goette A. Pathophysiological mechanisms of atrial fibrillation: a translational appraisal. Physiol Rev. 2011;91:265-325.

33. Fuster V, Rydén LE, Cannom DS, Crijns HJ, Curtis AB, Ellenbogen KA, et al $2011 \mathrm{ACCF} / \mathrm{AHA} / \mathrm{HRS}$ focused updates incorporated into the ACC/AHA/ESC 2006 guidelines for the management of patients with atrial fibrillation: a report of the American College of Cardiology Foundation/American Heart Association Task Force on Practice Guidelines developed in partnership with the European Society of Cardiology and in collaboration with the European Heart Rhythm Association and the Heart Rhythm Society. J Am Coll Cardiol. 2011;57:e101-98.

34. Dixit S. Atrial fibrillation after major thoracic surgery: new insights into underlying mechanisms. J Am Coll Cardiol. 2009;54:2049-51.

35. Merritt RE, Shrager JB. Prophylaxis and management of atrial fibrillation after general thoracic surgery. Thorac Surg Clin. 2012;22:13-23.

36. Amar D, Roistacher N, Rusch VW, Leung DHY, Ginsburg I, Zhang H, et al Effects of diltiazem prophylaxis on the incidence and clinical outcome of atrial arrhythmias after thoracic surgery. J Thorac Cardiovasc Surg. 2000; 120:790-8. 
37. Van Mieghem W, Tits G, Demuynck K, Lacquet L, Deneffe G, Tjandra-Maga T, et al. Verapamil as prophylactic treatment for atrial fibrillation after lung operations. Ann Thorac Surg. 1996;61:1083-6.

38. Anderson JL, Halperin JL, Albert NM, Bozkurt B, Brindis RG, Curtis LH, et al. Management of patients with atrial fibrillation (compilation of 2006 ACCF/ $\mathrm{AHA} / \mathrm{ESC}$ and $2011 \mathrm{ACCF} / \mathrm{AHA} / \mathrm{HRS}$ recommendations): a report of the American College of Cardiology/American Heart Association Task Force on Practice Guidelines. J Am Coll Cardiol. 2013;61:1935-44.

39. Neumar RW, Otto CW, Link MS, Kronick SL, Shuster M, Callaway CW, et al. Part 8: Adult advanced cardiovascular life support 2010 American Heart Association guidelines for cardiopulmonary resuscitation and emergency cardiovascular care. Circulation. 2010;122(18 Suppl 3):S729-67.

40. Van Mieghem W, Coolen L, Malysse I, Lacquet LM, Deneffe GJ, Demedts MG. Amiodarone and the development of ARDS after lung surgery. Chest. 1994; 105:1642-5.

41. Camm J. Antiarrhythmic drugs for the maintenance of sinus rhythm: risks and benefits. Int J Cardiol. 2012;155:362-71.

42. Dorian P. Antiarrhythmic action of beta-blockers: potential mechanisms. J Cardiovasc Pharmacol Ther. 2005;10:S15-22.

43. Fuster V, Rydén LE, Cannom DS, Crijns HJ, Curtis AB, Ellenbogen KA, et al. American College of Cardiology/American Heart Association Task Force on Practice Guidelines, European Society of Cardiology Committee for Practice Guidelines, European Heart Rhythm Association, Heart Rhythm Society, ACC/AHA/ESC 2006 guidelines for the management of patients with atrial fibrillation: a report of the American College of Cardiology/American Heart Association Task Force on Practice Guidelines and the European Society of Cardiology Committee for Practice Guidelines (Writing Committee to Revise the 2001 Guidelines for the Management of Patients With Atrial Fibrillation): developed in collaboration with the European Heart Rhythm Association and the Heart Rhythm Society. Circulation. 2006;114:e257-354.

44. Van Gelder IC, Groenveld HF, Crijns HJGM, Tuininga YS, Tijssen JGP, Alings AM, et al; RACE II Investigators. Lenient versus strict rate control in patients with atrial fibrillation. N Engl J Med. 2010;362:1363-73.

45. Olshansky B, Rosenfeld LE, Warner AL, Solomon AJ, O'Neill G, Sharma A, et al; AFFIRM Investigators. The Atrial Fibrillation Follow-up Investigation of Rhythm Management (AFFIRM) study: approaches to control rate in atrial fibrillation. J Am Coll Cardiol. 2004;43:1201-8.

46. Farshi R, Kistner D, Sarma JS, Longmate JA, Singh BN. Ventricular rate control in chronic atrial fibrillation during daily activity and programmed exercise: a crossover open-label study of 5 drug regimens. J Am Coll Cardiol. 1999;33: 304-10.

47. Abrams J, Allen J, Allin D, Anderson J, Anderson S, Blanski L, et al. Efficacy and safety of esmolol vs propranolol in the treatment of supraventricular tachyarrhythmias: a multicenter double-blind clinical trial. Am Heart J. 1985;110:913-22.

48. Echizen H, Eichelbaum M. Clinical pharmacokinetics of verapamil, nifedipine and diltiazem. Clin Pharmacokinet. 1986;11:425-49.

49. Siu C-W, Lau C-P, Lee W-L, Lam K-F, Tse H-F. Intravenous diltiazem is superior to intravenous amiodarone or digoxin for achieving ventricular rate control in patients with acute uncomplicated atrial fibrillation. Crit Care Med. 2009;37: 2174-9; quiz 2180.

50. Delle Karth G, Geppert A, Neunteufl T, Priglinger U, Haumer M, Gschwandtner M, et al. Amiodarone versus diltiazem for rate control in critically ill patients with atrial tachyarrhythmias. Crit Care Med. 2001;29:1149-53.

51. Gheorghiade M, Adams KF, Colucci WS. Digoxin in the management of cardiovascular disorders. Circulation. 2004;109:2959-64.

52. Jordaens L, Trouerbach J, Calle P, Tavernier R, Derycke E, Vertongen P, et al Conversion of atrial fibrillation to sinus rhythm and rate control by digoxin in comparison to placebo. Eur Heart J. 1997;18:643-8.

53. Intravenous digoxin in acute atrial fibrillation. Results of a randomized, placebo-controlled multicentre trial in 239 patients. The Digitalis in Acute Atrial Fibrillation (DAAF) Trial Group. Eur Heart J. 1997;18:649-54.

54. Gheorghiade M, Fonarow GC, Van Veldhuisen DJ, Cleland JGF, Butler J, Epstein AE, et al. Lack of evidence of increased mortality among patients with atrial fibrillation taking digoxin: findings from post hoc propensity-matched analysis of the AFFIRM trial. Eur Heart J. 2013; 34:1489-97.

55. Balser JR. The rational use of intravenous amiodarone in the perioperative period. Anesthesiology. 1997;86:974-87.

56. Sanoski CA. Antiarrhythmic agents. In: Wiggins BS, Sanoski CA, eds. Emergency Cardiovascular Pharmacotherapy: A Point-of-Care Guide. Bethesda, Md: American Society of Health-System Pharmacists; 2012:61-8.
57. Tse HF, Lam YM, Lau CP, Cheung BM, Kumana CR. Comparison of digoxin versus low-dose amiodarone for ventricular rate control in patients with chronic atrial fibrillation. Clin Exp Pharmacol Physiol. 2001;28:446-50.

58. Roden DM, Wood A, Wilkinson GR, Woosley RL. Disposition kinetics of encainide and metabolites. Am J Cardiol. 1986;58:4C-9C.

59. Tamargo J, Capucci A, Mabo P. Safety of flecainide. Drug Saf. 2012;35: 273-89.

60. Echt DS, Liebson PR, Mitchell LB, Peters RW, Obias-Manno D, Barker AH, et al. Mortality and morbidity in patients receiving encainide, flecainide, or placebo. N Engl J Med. 1991;324:781-8.

61. Naccarelli GV, Wolbrette DL, Luck JC. Proarrhythmia. Med Clin North Am. 2001;85:503-26; xii.

62. Wu JY, Lipsius SL. Effects of extracellular $\mathrm{Mg}^{2+}$ on T- and L-type $\mathrm{Ca}^{2+}$ currents in single atrial myocytes. Am J Physiol. 1990;259:H1842-50.

63. Iseri LT, Allen BJ, Ginkel ML, Brodsky MA. Ionic biology and ionic medicine in cardiac arrhythmias with particular reference to magnesium. Am Heart J. 1992:123:1404-9.

64. Rasmussen HS, Larsen OG, Meier K, Larsen J. Hemodynamic effects of intravenously administered magnesium on patients with ischemic heart disease. Clin Cardiol. 1988;11:824-8.

65. Ho KM, Sheridan DJ, Paterson T. Use of intravenous magnesium to treat acute onset atrial fibrillation: a meta-analysis. Heart. 2007;93:1433-40.

66. Carmeliet E. Voltage- and time-dependent block of the delayed $\mathrm{K}^{+}$ current in cardiac myocytes by dofetilide. J Pharmacol Exp Ther. 1992;262: 809-17.

67. Mounsey JP, DiMarco JP. Cardiovascular drugs. Dofetilide. Circulation. 2000; 102:2665-70.

68. Singh S, Zoble RG, Yellen L, Brodsky MA, Feld GK. Efficacy and safety of oral dofetilide in converting to and maintaining sinus rhythm in patients with chronic atrial fibrillation or atrial flutter the symptomatic atrial fibrillation investigative research on dofetilide (SAFIRE-D) study. Circulation. 2000; 102:2385-90.

69. Pedersen OD, Bagger H, Keller N, Marchant B, Køber L, Torp-Pedersen C. Efficacy of dofetilide in the treatment of atrial fibrillation-flutter in patients with reduced left ventricular function: a Danish investigations of arrhythmia and mortality on dofetilide (diamond) substudy. Circulation. 2001;104: 292-6.

70. Torp-Pedersen C, Møller M. Dofetilide in patients with congestive heart failure and left ventricular dysfunction. Danish Investigators of Arrhythmia and Mortality on Dofetilide Study Group. N Engl J Med. 1999;341:857-65.

71. Adlan AM, Lip GY. Benefit-risk assessment of dronedarone in the treatment of atrial fibrillation. Drug Saf. 2013;36:93-110.

72. Køber L, Torp-Pedersen C, McMurray JJV, Gøtzsche O, Lévy S, Crijns H, et al; Dronedarone Study Group. Increased mortality after dronedarone therapy for severe heart failure. $N$ Engl J Med. 2008;58:2678-87.

73. Connolly SJ, Camm AJ, Halperin JL, Joyner C, Alings M, Amerena J, et al; PALLAS Investigators. Dronedarone in high-risk permanent atrial fibrillation. N Engl J Med. 2011;365:2268-76.

74. Lee KS. Ibutilide, a new compound with potent class III antiarrhythmic activity, activates a slow inward $\mathrm{Na}^{+}$current in guinea pig ventricular cells. J Pharmacol Exp Ther. 1992;262:99-108.

75. Yang T, Snyders DJ, Roden DM. Ibutilide, a methanesulfonanilide antiarrhythmic, is a potent blocker of the rapidly activating delayed rectifier $\mathrm{K}^{+}$current (IKr) in AT-1 cells. Concentration-, time-, voltage-, and use-dependent effects. Circulation. 1995;91:1799-806.

76. Stambler BS, Wood MA, Ellenbogen KA, Perry KT, Wakefield LK, VanderLugt JT. Efficacy and safety of repeated intravenous doses of ibutilide for rapid conversion of atrial flutter or fibrillation. Ibutilide Repeat Dose Study Investigators. Circulation. 1996;94:1613-21.

77. VanderLugt JT, Mattioni T, Denker S, Torchiana D, Ahern T, Wakefield LK, et al. Efficacy and safety of ibutilide fumarate for the conversion of atrial arrhythmias after cardiac surgery. Circulation. 1999;100:369-75.

78. Slavik RS, Tisdale JE, Borzak S. Pharmacologic conversion of atrial fibrillation: a systematic review of available evidence. Prog Cardiovasc Dis. 2001; 44:121-52.

79. Seipel L, Breithardt G. Propafenone-a new antiarrhythmic drug. Eur Heart J. 1980;1:309-13.

80. Seki A, Hagiwara N, Kasanuki H. Effects of propafenone on K currents in human atrial myocytes. Br J Pharmacol. 1999;126:1153-62.

81. Pritchett EL, McCarthy EA, Wilkinson WE. Propafenone treatment of symptomatic paroxysmal supraventricular arrhythmias. A randomized, placebo- 
controlled, crossover trial in patients tolerating oral therapy. Ann Intern Med. 1991;114:539-44.

82. Boriani G, Biffi M, Capucci A, Botto GL, Broffoni T, Rubino I, et al. Oral propafenone to convert recent-onset atrial fibrillation in patients with and without underlying heart disease. A randomized, controlled trial. Ann Intern Med. 1997;126:621-5

83. Singh BN, Nademanee K. Sotalol: a beta blocker with unique antiarrhythmic properties. Am Heart J. 1987;114:121-39.

84. Carmeliet E. Electrophysiologic and voltage clamp analysis of the effects of sotalol on isolated cardiac muscle and Purkinje fibers. J Pharmacol Exp Ther. $1985 ; 232: 817-25$

85. Benditt DG, Williams JH, Jin J, Deering TF, Zucker R, Browne K, et al. Maintenance of sinus rhythm with oral d,l-sotalol therapy in patients with symptomatic atrial fibrillation and/or atrial flutter. d,l-Sotalol Atrial Fibrillation/Flutter Study Group. Am J Cardiol. 1999;84:270-7.

86. Lafuente-Lafuente C, Mouly S, Longas-Tejero MA, Bergmann JF. Antiarrhythmics for maintaining sinus rhythm after cardioversion of atrial fibrillation. Cochrane Database Syst Rev. 2007;CD005049.

87. Auer J, Weber T, Berent R, Puschmann R, Hartl P, Ng C-K, et al. Study of prevention of postoperative atrial fibrillation. A comparison between oral antiarrhythmic drugs in the prevention of atrial fibrillation after cardiac surgery: the pilot study of prevention of postoperative atrial fibrillation (SPPAF), a randomized, placebo-controlled trial. Am Heart J. 2004;147:636-43.

88. Nattel S, Quantz MA. Pharmacological response of quinidine induced early afterdepolarisations in canine cardiac Purkinje fibres: insights into underlying ionic mechanisms. Cardiovasc Res. 1988;22:808-17.

89. Nenov NI, Crumb WJ, Pigott JD, Harrison LH, Clarkson CW. Quinidine interactions with human atrial potassium channels: developmental aspects. Circ Res. 1998;83:1224-31.

90. Lafuente-Lafuente C, Mouly S, Longás-Tejero MA, Mahé I, Bergmann J-F. Antiarrhythmic drugs for maintaining sinus rhythm after cardioversion of atrial fibrillation: a systematic review of randomized controlled trials. Arch Intern Med. 2006; 166:719-28.

91. Yancy CW, Jessup M, Bozkurt B, Butler J, Casey DE, Drazner MH, et al. 2013 ACCF/AHA guideline for the management of heart failure: a report of the American College of Cardiology Foundation/American Heart Association Task Force on Practice Guidelines. Circulation. 2013;128:e240-327.

92. Smith TW, Haber E. Digoxin intoxication: the relationship of clinical presentation to serum digoxin concentration. J Clin Invest. 1970;49:2377-86.

93. Burton ME. Applied Pharmacokinetics \& Pharmacodynamics. Philadelphia, Pa: Lippincott Williams \& Wilkins; 2006.

94. Tisdale JE, Wroblewski HA, Wall DS, Rieger KM, Hammoud ZT, Young JV, et al. A randomized trial evaluating amiodarone for prevention of atrial fibrillation after pulmonary resection. Ann Thorac Surg. 2009;88:886-93; discussion 894-5.

95. Barbetakis N, Vassiliadis M. Is amiodarone a safe antiarrhythmic to use in supraventricular tachyarrhythmias after lung cancer surgery? BMC Surg. 2004;4:7.

96. Riber LP, Christensen TD, Jensen HK, Hoejsgaard A, Pilegaard HK. Amiodarone significantly decreases atrial fibrillation in patients undergoing surgery for lung cancer. Ann Thorac Surg. 2012;94:339-44; discussion 345-6.

97. Tisdale JE. Ventricular arrhythmias. In: Tisdale JE, Miller DA, eds. DrugInduced Diseases. Bethesda, Md: American Society of Health-System Pharmacists; 2010:485-515

98. Trinkley KE, Page RL, Lien H, Yamanouye K, Tisdale JE. QT interval prolongation and the risk of torsades de pointes: essentials for clinicians. Curr Med Res Opin. 2013;29:1719-26.

99. Tisdale JE, Padhi ID, Goldberg AD, Silverman NA, Webb CR, Higgins RS, et al. A randomized, double-blind comparison of intravenous diltiazem and digoxin for atrial fibrillation after coronary artery bypass surgery. Am Heart J. 1998;135:739-47.

100. Nattel S, Rangno RE, Van Loon G. Mechanism of propranolol withdrawal phenomena. Circulation. 1979:59:1158-64.

101. Jakobsen C-J, Bille S, Ahlburg P, Rybro L, Hjortholm K, Bay Andresen E. Perioperative metoprolol reduces the frequency of atrial fibrillation after thoracotomy for lung resection. J Cardiothorac Vasc Anesth. 1997;11:746-51.

102. Bayliff CD, Massel DR, Inculet RI, Malthaner RA, Quinton SD, Powell FS, et al. Propranolol for the prevention of postoperative arrhythmias in general thoracic surgery. Ann Thorac Surg. 1999;67:182-6.

103. Burgess DC, Kilborn MJ, Keech AC. Interventions for prevention of postoperative atrial fibrillation and its complications after cardiac surgery: a meta-analysis. Eur Heart J. 2006;27:2846-57.
104. Rena O, Papalia E, Oliaro A, Casadio C, Ruffini E, Filosso PL, et al. Supraventricular arrhythmias after resection surgery of the lung. Eur J Cardiothorac Surg. 2001;20:688-93.

105. Rostron A, Sanni A, Dunning J. Does magnesium prophylaxis reduce the incidence of atrial fibrillation following coronary bypass surgery? Interact Cardiovasc Thorac Surg. 2005;4:52-8.

106. Ritchie AJ, Tolan M, Whiteside M, McGuigan JA, Gibbons JR. Prophylactic digitalization fails to control dysrhythmia in thoracic esophageal operations. Ann Thorac Surg. 1993;55:86-8.

107. Kaiser A, Zünd G, Weder W, Largiadèr F. Preventive digitalis therapy in open thoracotomy. Helv Chir Acta. 1994;60:913-7.

108. Amar D, Roistacher N, Burt ME, Rusch VW, Bains MS, Leung DH, et al Effects of diltiazem versus digoxin on dysrhythmias and cardiac function after pneumonectomy. Ann Thorac Surg. 1997;63:1374-81; discussion 1381-2.

109. See VY, Roberts-Thomson KC, Stevenson WG, Camp PC, Koplan BA. Atria arrhythmias after lung transplantation: epidemiology, mechanisms at electrophysiology study, and outcomes. Circ Arrhythm Electrophysiol. 2009;2: 504-10.

110. Mason DP, Marsh DH, Alster JM, Murthy SC, McNeill AM, Budev MM, et al Atrial fibrillation after lung transplantation: timing, risk factors, and treatment. Ann Thorac Surg. 2007;84:1878-84.

111. Dizon JM, Chen K, Bacchetta M, Argenziano M, Mancini D, Biviano A, et al. A comparison of atrial arrhythmias after heart or double-lung transplantation at a single center: insights into the mechanism of post-operative atrial fibrillation. $J$ Am Coll Cardiol. 2009;54:2043-8.

112. Wijeysundera DN, Beattie WS, Rao V, Karski J. Calcium antagonists reduce cardiovascular complications after cardiac surgery: a meta-analysis. $J$ Am Coll Cardiol. 2003;41:1496-505

113. Khalil MA, Al-Agaty AE, Ali WG, Abdel Azeem MS. A comparative study between amiodarone and magnesium sulfate as antiarrhythmic agents for prophylaxis against atrial fibrillation following lobectomy. J Anesth. 2013;27:56-61.

114. Lanza LA, Visbal AI, DeValeria PA, Zinsmeister AR, Diehl NN, Trastek VF. Low-dose oral amiodarone prophylaxis reduces atrial fibrillation after pulmonary resection. Ann Thorac Surg. 2003;75:223-30; discussion 230.

115. Amar D, Zhang H, Heerdt PM, Park B, Fleisher M, Thaler HT. Statin use is associated with a reduction in atrial fibrillation after noncardiac thoracic surgery in dependent of C-reactive protein. Chest. 2005;128:3421-7.

116. Fauchier L, Pierre B, de Labriolle A, Grimard C, Zannad N, Babuty D. Antiarrhythmic effect of statin therapy and atrial fibrillation a meta-analysis of randomized controlled trials. J Am Coll Cardiol. 2008;51:828-35.

117. Chopra V, Wesorick DH, Sussman JB, Greene T, Rogers M, Froehlich JB, et al Effect of perioperative statins on death, myocardial infarction, atrial fibrillation, and length of stay: a systematic review and meta-analysis. Arch Surg. 2012;147: 181-9.

118. Johnson WD, Ganjoo AK, Stone CD, Srivyas RC, Howard M. The left atria appendage: our most lethal human attachment! Surgical implications. Eur $J$ Cardiothorac Surg. 2000;17:718-22.

119. POISE Study Group, Devereaux PJ, Yang H, Yusuf S, Guyatt G, Leslie K, et al Effects of extended-release metoprolol succinate in patients undergoing noncardiac surgery (POISE trial): a randomised controlled trial. Lancet. 2008; 371:1839-47.

120. Isiadinso I, Meshkov AB, Gaughan J, Sandhu P, Lim S, Cordova F, et al Atrial arrhythmias after lung and heart-lung transplant: effects on short-term mortality and the influence of amiodarone. J Heart Lung Transplant. 2011 30:37-44.

121. Li J, Solus J, Chen Q, Rho YH, Milne G, Stein CM, et al. Role of inflammation and oxidative stress in atrial fibrillation. Heart Rhythm. 2010;7:438-44.

122. Gammie JS, Haddad M, Milford-Beland S, Welke KF, Ferguson TB, O’Brien SM, et al. Atrial fibrillation correction surgery: lessons from the Society of Thoracic Surgeons National Cardiac Database. Ann Thorac Surg. 2008;85:909-14.

123. Doukas G, Samani NJ, Alexiou C, Oc M, Chin DT, Stafford PG, et al. Left atria radiofrequency ablation during mitral valve surgery for continuous atrial fibrillation: a randomized controlled trial. JAMA. 2005;294:2323-9.

124. Reddy VY, Doshi SK, Sievert H, Buchbinder M, Neuzil P, Huber K, et al; PROTECT AF Investigators. Percutaneous left atrial appendage closure for stroke prophylaxis in patients with atrial fibrillation: 2.3-Year follow-up of the PROTECT AF (Watchman Left Atrial Appendage System for Embolic Protection in Patients with Atrial Fibrillation) trial. Circulation. 2013;127:720-9.

125. Bobbio A, Caporale D, Internullo E, Ampollini L, Bettati S, Rossini E, et al Postoperative outcome of patients undergoing lung resection presenting with 
new-onset atrial fibrillation managed by amiodarone or diltiazem. Eur J Cardiothorac Surg. 2007;31:70-4.

126. Lee JK, Klein GJ, Krahn AD, Yee R, Zarnke K, Simpson C, et al. Rate-control versus conversion strategy in postoperative atrial fibrillation: a prospective, randomized pilot study. Am Heart J. 2000;140:871-7.

127. Soucier R, Silverman D, Abordo M, Jaagosild P, Abiose A, Madhusoodanan KP, et al. Propafenone versus ibutilide for post operative atrial fibrillation following cardiac surgery: neither strategy improves outcomes compared to rate control alone (the PIPAF study). Med Sci Monit. 2003;9:PI19-23.

128. Valentine RJ, Rosen SF, Cigarroa JE, Jackson MR, Modrall JG, Clagett GP. The clinical course of new-onset atrial fibrillation after elective aortic operations. $J$ Am Coll Surg. 2001;193:499-504.

129. Wyse DG, Waldo AL, DiMarco JP, Domanski MJ, Rosenberg Y, Schron EB, et al. A comparison of rate control and rhythm control in patients with atrial fibrillation,. N Engl J Med. 2002;347:1825-33.

130. Van Gelder IC, Hagens VE, Bosker HA, Kingma JH, Kamp O, Kingma T, et al. A comparison of rate control and rhythm control in patients with recurrent persistent atrial fibrillation,. N Engl J Med. 2002;347:1834-40.

131. Roy D, Talajic M, Nattel S, Wyse DG, Dorian P, Lee KL, et al; Atrial Fibrillation and Congestive Heart Failure Investigators. Rhythm control versus rate control for atrial fibrillation and heart failure. N Engl J Med. 2008;358: 2667-77.

132. Hohnloser SH, Kuck KH, Lilienthal J. Rhythm or rate control in atrial fibrillation-Pharmacological Intervention in Atrial Fibrillation (PIAF): a randomised trial. Lancet. 2000;356:1789-94

133. Carlsson J, Miketic S, Windeler J, Cuneo A, Haun S, Micus S, et al; STAF Investigators. Randomized trial of rate-control versus rhythm-control in persistent atrial fibrillation: the Strategies of Treatment of Atrial Fibrillation (STAF) study. J Am Coll Cardiol. 2003;41:1690-6.

134. Jenkins LS, Brodsky M, Schron E, Chung M, Rocco T Jr, Lader E, et al. Quality of life in atrial fibrillation: The Atrial Fibrillation Follow-up Investigation of Rhythm Management (AFFIRM) study. Am Heart J. 2005;149: 112-20.

135. Corley SD, Epstein AE, DiMarco JP, Domanski MJ, Geller N, Greene HL, et al; AFFIRM Investigators. Relationships between sinus rhythm, treatment, and survival in the Atrial Fibrillation Follow-Up Investigation of Rhythm Management (AFFIRM) Study. Circulation. 2004;109:1509-13.

136. Chung MK, Shemanski L, Sherman DG, Greene HL, Hogan DB, Kellen JC, et al; AFFIRM Investigators. Functional status in rate- versus rhythm-control strategies for atrial fibrillation: results of the Atrial Fibrillation Follow-Up Investigation of Rhythm Management (AFFIRM) functional status substudy. J Am Coll Cardiol. 2005;46:1891-9.

137. Ciriaco P, Mazzone P, Canneto B, Zannini P. Supraventricular arrhythmia following lung resection for non-small cell lung cancer and its treatment with amiodarone. Eur J Cardiothorac Surg. 2000;18:12-6.

138. Strasberg B, Arditti A, Sclarovsky S, Lewin RF, Buimovici B, Agmon J. Efficacy of intravenous amiodarone in the management of paroxysmal or new atrial fibrillation with fast ventricular response. Int J Cardiol. 1985;7:47-58.

139. Faniel R, Schoenfeld P. Efficacy of i.v. amiodarone in converting rapid atrial fibrillation and flutter to sinus rhythm in intensive care patients. Eur Heart J. $1983 ; 4: 180-5$

140. Kumar A. Intravenous amiodarone for therapy of atrial fibrillation and flutter in critically ill patients with severely depressed left ventricular function. South Med J. 1996;89:779-85.

141. Hilleman DE, Hunter CB, Mohiuddin SM, Maciejewski S. Pharmacologic management of atrial fibrillation following cardiac surgery. Am J Cardiovasc Drugs. 2005;5:361-9.

142. Hilleman DE, Spinler SA. Conversion of recent-onset atrial fibrillation with intravenous amiodarone: a meta-analysis of randomized controlled trials. Pharmacotherapy. 2002;22:66-74.

143. Reisinger J, Gatterer E, Lang W, Vanicek T, Eisserer G, Bachleitner T, et al. Flecainide versus ibutilide for immediate cardioversion of atrial fibrillation of recent onset. Eur Heart J. 2004;25:1318-24.

144. Di Biasi P, Scrofani R, Paje A, Cappiello E, Mangini A, Santoli C. Intravenous amiodarone vs propafenone for atrial fibrillation and flutter after cardiac operation. Eur J Cardiothorac Surg. 1995;9:587-91.

145. Geelen P, O'Hara GE, Roy N, Talajic M, Roy D, Plante S, et al. Comparison of propafenone versus procainamide for the acute treatment of atrial fibrillation after cardiac surgery. Am J Cardiol. 1999;84:345-7; A8-9.

146. McNamara RL, Tamariz LJ, Segal JB, Bass EB. Management of atrial fibrillation: review of the evidence for the role of pharmacologic therapy, electrical cardioversion, and echocardiography. Ann Intern Med. 2003;139: 1018-33.

147. Camm AJ, Lip GYH, De Caterina R, Savelieva I, Atar D, Hohnloser SH, et al; ESC Committee for Practice Guidelines (CPG). 2012 focused update of the ESC guidelines for the management of atrial fibrillation: an update of the 2010 ESC guidelines for the management of atrial fibrillation. Developed with the special contribution of the European Heart Rhythm Association. Eur Heart J. 2012;33:2719-47.

148. Karthik S, Musleh G, Grayson AD, Keenan DJ, Pullan DM, Dihmis WC, et al. Coronary surgery in patients with peripheral vascular disease: Effect of avoiding cardiopulmonary bypass. Ann Thorac Surg. 2004;77:1245-9.

149. Seguin P, Signouret T, Laviolle B, Branger B, Malledant Y. Incidence and risk factors of atrial fibrillation in a surgical intensive care unit. Crit Care Med. 2004;32:722-6.

150. Black IW, Fatkin D, Sagar KB, Khandheria BK, Leung DY, Galloway JM, et al. Exclusion of atrial thrombus by transesophageal echocardiography does not preclude embolism after cardioversion of atrial fibrillation. A multicenter study. Circulation. 1994;89:2509-13.

151. Klein AL, Grimm RA, Murray RD, Apperson-Hansen C, Asinger RW, Black IW, et al; Assessment of Cardioversion Using Transesophageal Echocardiography Investigators. Use of transesophageal echocardiography to guide cardioversion in patients with atrial fibrillation. N Engl J Med. 2001;344:1411-20.

152. Black IW, Hopkins AP, Lee L, Walsh WF. Evaluation of transesophageal echocardiography before cardioversion of atrial fibrillation and flutter in nonanticoagulated patients. Am Heart J. 1993;126:375-81.

153. Romero J, Husain SA, Kelesidis I, Sanz J, Medina HM, Garcia MJ. Detection of left atrial appendage thrombus by cardiac computed tomography in patients with atrial fibrillation: a meta-analysis. Circ Cardiovasc Imaging. 2013;6: 185-94.

154. Calkins H, Kuck KH, Cappato R, Brugada J, Camm AJ, Chen S-A, et al. 2012 HRS/EHRA/ECAS expert consensus statement on catheter and surgical ablation of atrial fibrillation: recommendations for patient selection, procedural techniques, patient management and follow-up, definitions, endpoints, and research trial design: a report of the Heart Rhythm Society (HRS) Task Force on Catheter and Surgical Ablation of Atrial Fibrillation. Developed in partnership with the European Heart Rhythm Association (EHRA), a registered branch of the European Society of Cardiology (ESC) and the European Cardiac Arrhythmia Society (ECAS); and in collaboration with the American College of Cardiology (ACC), American Heart Association (AHA), the Asia Pacific Heart Rhythm Society (APHRS), and the Society of Thoracic Surgeons (STS). Endorsed by the governing bodies of the American College of Cardiology Foundation, the American Heart Association, the European Cardiac Arrhythmia Society, the European Heart Rhythm Association, the Society of Thoracic Surgeons, the Asia Pacific Heart Rhythm Society, and the Heart Rhythm Society. Heart Rhythm. 2012;9:632-96.

155. Reddy VY, Möbius-Winkler S, Miller MA, Neuzil P, Schuler G, Wiebe J, et al. Left atrial appendage closure with the Watchman device in patients with a contraindication for oral anticoagulation: the ASAP study (ASA Plavix Feasibility Study With Watchman Left Atrial Appendage Closure Technology). J Am Coll Cardiol. 2013;61:2551-6.

156. Sherwood MW, Douketis JD, Patel MR, Piccini JP, Hellkamp AS, Lokhnygina Y, et al; ROCKET AF Investigators. Outcomes of temporary interruption of rivaroxaban compared with warfarin in patients with nonvalvular atrial fibrillation: results from the Rivaroxaban Once Daily, Oral, Direct Factor Xa Inhibition Compared With Vitamin K Antagonism for Prevention of Stroke and Embolism Trial in Atrial Fibrillation (ROCKET AF). Circulation. 2014; 129:1850-9.

157. Patel MR, Mahaffey KW, Garg J, Pan G, Singer DE, Hacke W, et al; ROCKET AF Investigators. Rivaroxaban versus warfarin in nonvalvular atrial fibrillation. N Engl J Med. 2011;365:883-91.

158. Kearon C, Hirsh J. Management of anticoagulation before and after elective surgery. N Engl J Med. 1997;336:1506-11.

159. Epstein AE, Alexander JC, Gutterman DD, Maisel W, Wharton JM American College of Chest Physicians. Anticoagulation: American College of Chest Physicians guidelines for the prevention and management of postoperative atrial fibrillation after cardiac surgery. Chest. 2005;128:24S-7S.

160. Douketis JD, Spyropoulos AC, Spencer FA, Mayr M, Jaffer AK, Eckman MH, et al. Perioperative management of antithrombotic therapy. Antithrombotic Therapy and Prevention of Thrombosis, 9th ed: American College of Chest Physicians Evidence-Based Clinical Practice Guidelines. Chest. 2012;141(2 Suppl):e326S-3250S. 
161. Hylek EM, Go AS, Chang Y, Jensvold NG, Henault LE, Selby JV, et al. Effect of intensity of oral anticoagulation on stroke severity and mortality in atrial fibrillation. N Engl J Med. 2003;349:1019-26.

162. Van Spall HG, Wallentin L, Yusuf S, Eikelboom JW, Nieuwlaat R, Yang S, et al. Variation in warfarin dose adjustment practice is responsible for differences in the quality of anticoagulation control between centers and countries: an analysis of patients receiving warfarin in the randomized evaluation of long-term anticoagulation therapy (RE-LY) trial. Circulation. 2012;126:2309-16.

163. Ageno W, Gallus AS, Wittkowsky A, Crowther M, Hylek EM, Palareti G American College of Chest Physicians. Oral anticoagulant therapy: Antithrombotic Therapy and Prevention of Thrombosis, 9th ed: American College of Chest Physicians Evidence-Based Clinical Practice Guidelines. Chest. 2012;141(2 Suppl):e44S-88S.

164. Pengo V, Barbero F, Biasiolo A, Pegoraro C, Cucchini U, Iliceto S. A comparison between six- and four-week intervals in surveillance of oral anticoagulant treatment. Am J Clin Pathol. 2003;120:944-7.

165. Schulman S, Parpia S, Stewart C, Rudd-Scott L, Julian JA, Levine M. Warfarin dose assessment every 4 weeks versus every 12 weeks in patients with stable international normalized ratios: a randomized trial. Ann Intern Med. 2011;155: 653-9; W201-W203.

166. Bateman BT, Schumacher HC, Wang S, Shaefi S, Berman MF. Perioperative acute ischemic stroke in noncardiac and nonvascular surgery: incidence, risk factors, and outcomes. Anesthesiology. 2009;110:231-8.

167. Botto F, Alonso-Coello P, Chan MTV, Villar JC, Xavier D, Srinathan S, et al; Vascular events In noncardiac Surgery patIents cOhort evaluatioN (VISION) Writing Group, on behalf of The Vascular events In noncardiac Surgery patIents cOhort evaluatioN (VISION) Investigators. Appendix 1. The Vascular events In noncardiac Surgery patIents cOhort evaluatioN (VISION) Study Investigators Writing Group, Appendix 2. The Vascular events In noncardiac Surgery patIents cOhort evaluatioN Operations Committee, Vascular events In noncardiac Surgery patIents cOhort evaluatioN VISION Study Investigators. Myocardial injury after noncardiac surgery: a large, international, prospective cohort study establishing diagnostic criteria, characteristics, predictors, and 30-day outcomes. Anesthesiology. 2014;120:564-78.

168. Amar D. Perioperative atrial tachyarrhythmias. Anesthesiology. 2002;97:1618-23.

169. Ng JL, Chan MT, Gelb AW. Perioperative stroke in noncardiac, nonneurosurgical surgery. Anesthesiology. 2011;115:879-90.

170. Verhaert D, Puwanant S, Gillinov AM, Klein AL. Atrial fibrillation after open heart surgery: how safe is early conversion without anticoagulation? J Am Soc Echocardiogr. 2009;22:212.e1-3.

171. White HD, Gruber M, Feyzi J, Kaatz S, Tse H-F, Husted S, et al. Comparison of outcomes among patients randomized to warfarin therapy according to anticoagulant control: results from SPORTIF III and V. Arch Intern Med. 2007;167:239-45.

172. Adjusted-dose warfarin versus low-intensity, fixed-dose warfarin plus aspirin for high-risk patients with atrial fibrillation: Stroke Prevention in Atrial Fibrillation III randomised clinical trial. Lancet. 1996;348:633-8.

173. Pisters R, Lane DA, Nieuwlaat R, de Vos CB, Crijns HJ, Lip GY. A novel user-friendly score (HAS-BLED) to assess 1-year risk of major bleeding in patients with atrial fibrillation: the Euro Heart Survey. Chest. 2010;138: 1093-100.

174. Gage BF, Yan Y, Milligan PE, Waterman AD, Culverhouse R, Rich MW, et al. Clinical classification schemes for predicting hemorrhage: results from the National Registry of Atrial Fibrillation (NRAF). Am Heart J. 2006;151:713-9.

175. Fang MC, Go AS, Chang Y, Borowsky LH, Pomernacki NK, Udaltsova N, et al. A new risk scheme to predict warfarin-associated hemorrhage: the ATRIA
(Anticoagulation and Risk Factors in Atrial Fibrillation) study. J Am Coll Cardiol. 2011:58:395-401.

176. Shah M, Avgil Tsadok M, Jackevicius CA, Essebag V, Eisenberg MJ, Rahme E et al. Warfarin use and the risk for stroke and bleeding in patients with atrial fibrillation undergoing dialysis. Circulation. 2014;129:1196-203.

177. Risk factors for stroke and efficacy of antithrombotic therapy in atrial fibrillation. Analysis of pooled data from 5 randomized controlled trials. Arch Intern Med. 1994;154:1449-57.

178. Warfarin versus aspirin for prevention of thromboembolism in atrial fibrillation: Stroke Prevention in Atrial Fibrillation II Study. Lancet. 1994;343: 687-91.

179. Ma Q. Development of oral anticoagulants. Br J Clin Pharmacol. 2007;64:263-5.

180. Paikin JS, Eikelboom JW, Cairns JA, Hirsh J. New antithrombotic agents-insights from clinical trials. Nat Rev Cardiol. 2010;7:498-509.

181. Stangier J. Clinical pharmacokinetics and pharmacodynamics of the ora direct thrombin inhibitor dabigatran etexilate. Clin Pharmacokinet. 2008;47: 285-95.

182. Perzborn E, Roehrig S, Straub A, Kubitza D, Misselwitz F. The discovery and development of rivaroxaban, an oral, direct factor Xa inhibitor. Nat Rev Drug Discov. 2011;10:61-75.

183. Connolly SJ, Ezekowitz MD, Yusuf S, Eikelboom J, Oldgren J, Parekh A, et al RE-LY Steering Committee and Investigators. Dabigatran versus warfarin in patients with atrial fibrillation. $N$ Engl J Med. 2009;361:1139-51.

184. Granger CB, Alexander JH, McMurray JJV, Lopes RD, Hylek EM Hanna M, et al; ARISTOTLE Committees and Investigators. Apixaban versus warfarin in patients with atrial fibrillation. $N$ Engl J Med. 2011; 365:981-92.

185. Eikelboom JW, Connolly SJ, Brueckmann M, Granger CB, Kappetein AP Mack MJ, et al; RE-ALIGN Investigators. Dabigatran versus warfarin in patients with mechanical heart valves. N Engl J Med. 2013;369:1206-14.

186. Amar D. Postoperative atrial fibrillation. Heart Dis. 2002;4:117-23.

187. Mitchell LB CCS Atrial Fibrillation Guidelines Committee. Canadian Cardiovascular Society atrial fibrillation guidelines 2010: prevention and treatment of atrial fibrillation following cardiac surgery. Can J Cardiol. 2011; 27:91-7.

188. Kowey PR, Stebbins D, Igidbashian L, Goldman SM, Sutter FP, Rials SJ, et al Clinical outcome of patients who develop PAF after CABG surgery. Pacing Clin Electrophysiol. 2001;24:191-3.

189. Izhar U, Ad N, Rudis E, Milgalter E, Korach A, Viola N, et al. When should we discontinue antiarrhythmic therapy for atrial fibrillation after coronary artery bypass grafting? A prospective randomized study. J Thorac Cardiovasc Surg. 2005;129:401-6.

190. Ahlsson A, Fengsrud E, Bodin L, Englund A. Postoperative atrial fibrillation in patients undergoing aortocoronary bypass surgery carries an eightfold risk of future atrial fibrillation and a doubled cardiovascular mortality. Eur J Cardiothorac Surg. 2010;37:1353-9.

191. Landymore RW, Howell F. Recurrent atrial arrhythmias following treatment for postoperative atrial fibrillation after coronary bypass operations. Eur J Cardiothorac Surg. 1991;5:436-9.

192. Yilmaz AT, Demírkiliç U, Arslan M, Kurulay E, Ozal E, Tatar H, et al. Longterm prevention of atrial fibrillation after coronary artery bypass surgery: comparison of quinidine, verapamil, and amiodarone in maintaining sinus rhythm. $J$ Card Surg. 1996;11:61-4.

193. Dunning J, Treasure T, Versteegh M, Nashef SAM. Guidelines on the prevention and management of de novo atrial fibrillation after cardiac and thoracic surgery. Eur J Cardiothorac Surg. 2006;30:852-72. 